\title{
RESIDUAL STRESS DETERMINATION FOR A FERRITIC STEEL WELD PLATE
}

\author{
D. -Q. Wang, \\ C. R. Hubbard \\ S. Spooner
}

Research sponsored by the Department of Energy

Assistant Secretary for Energy Efficiency

Office of Transportation Technologies

As part of the

High Temperature Materials Laboratory

Oak Ridge National Laboratory

Oak Ridge, Tennessee 37831-6064

Managed by

LOCKHEED MARTIN ENERGY RESEARCH CORP.

For the

U.S. DEPARTMENT OF ENERGY

Under contract DE-AC05-96OR22464 



\section{CONTENTS}

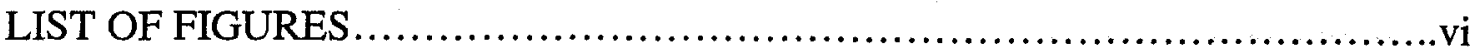

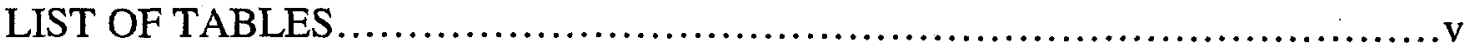

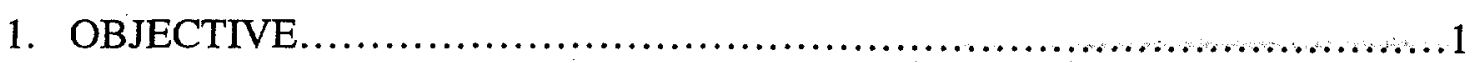

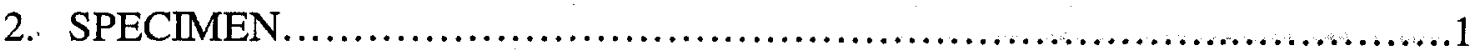

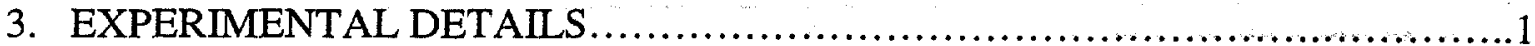

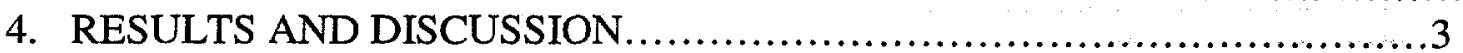

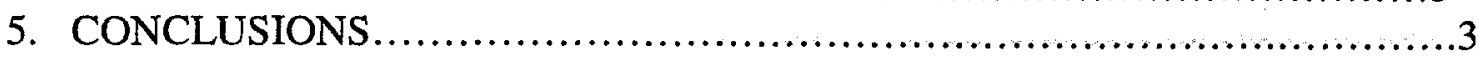




\section{LIST OF FIGURES}

Figure

Page

Fig. 1 (a) Comb-like d-zero specimen 5

(b) 3-D schematic drawing of the weld plate and strain/stress coordinate system

(c) Measurement locations at midsection

Fig. 2 2-Theta angle position variation at the lines in the vicinity of the weld for detector \#5

Fig. 3

Strain distribution at the mid-thickness line

Stress distribution at the mid-thickness line

Normal strain distribution in the vicinity of the weld

Fig. 5

Fig. 6

Fig. 7

Normal stress distribution in the vicinity of the weld

10

Transverse strain distribution in the vicinity of the weld

11

12

Transverse stress distribution in the vicinity of the weld 13

Fig. 8

Longitudinal strain distribution in the vicinity of the weld

14

Fig. 9

Longitudinal stress distribution in the vicinity of the weld Measured gauge volume for detector \#1

Fig. 11

Fig. 12

Measured gauge volume for detector \#2

Fig. 13

Measured gauge volume for detector \#3

17

Measured gauge volume for detector \#4

18

Fig. 14

Fig. 15

Measured gauge volume for detector \#5

19

Fig. 16

Measured gauge volume for detector \#6

20

Measured gauge volume for detector \#7 


\section{LIST OF TABLES}

Table

Table I. Pertinent experimental parameters

Page

Table II.

Experimental results

23

Table III. Derived strains and stresses

24

Table IV. Measured gauge volume size

54

59 


\section{OBJECTIVE}

The primary objective of this experiment is to demonstrate the capability of neutron diffraction technique to reproducibly map residual strains in a ferritic steel weld. The objective includes the identification of corrections for variations in metal composition due to the welding process which produces changes in lattice parameter that are not due to mechanical effects.

The second objective is to develop and demonstrate a "best practice" for neutron diffraction strain mapping of steel welds. The appropriate coordinate system for the measurement of a weld, which is strongly distorted from planar geometry, has to be defined. The coordinate system is important in determining the procedures for mounting and positioning of the weld so that mapping details, especially in regions of high gradients, can be conveniently inter-compared between laboratories.

\section{SPECIMEN}

The specimen is one of a set of VAMAS TWA $20^{*}$ round robin welds that have been circulated to the laboratories participating in this study. The weld specimens were provided by Rick Leggett of the Welding Institute (UK). The source of the round robin welds was a ferritic steel

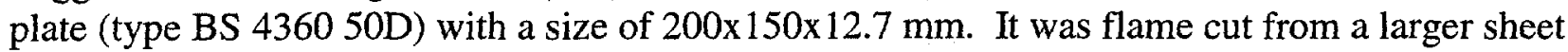
and the rough edges were then ground to produce reasonably smooth square edges. A $6 \mathrm{~mm} \mathrm{U-}$ groove was machined in the middle of the plate along its length to a depth of $8.5 \mathrm{~mm}$ leaving 4 $\mathrm{mm}$ of parent metal below. A 12-pass mechanized TIG weld was made in the groove using a travel speed of $240 \mathrm{~mm} / \mathrm{min}$. The plate was clamped for the first 10 passes but released for the last two. The resulting distortion left the plate bend at an angle of about 7 degrees (see Fig 1). Residual stresses at transverse extremities are not necessarily close to zero and the residual stress pattern is not totally symmetrical because of flame cutting variations and restraint during welding. A $3 \mathrm{~mm}$ section was then cut from each weld, which provided material for the determination of the zero-stress lattice parameter. Several partial cuts into this piece formed a comb-like configuration where each "tooth" was considered stress-free.

\section{EXPERIMENT DETAILS}

The residual stress instrument at Oak Ridge National Laboratory is a modification of a triple-axis spectrometer located at the HB-2 beam port of the High Flux Isotope Reactor (HFIR). The reactor is a beryllium reflected, light water moderated flux-trap research reactor operating at 85 MW. The core flux is $1.5 \times 10^{15}$ neutron $/ \mathrm{cm}^{2} / \mathrm{sec}$ which peaks in the beryllium reflector near the end of the radial beam tube. The monochromating crystal has a multiple-segment silicon

\footnotetext{
"Versailles Projects on Advanced Materials and Standards (VAMAS), Technical Working Group 20 (TWA-20).
} 
construction that permits elastic bending of the segments in the horizontal plane and vertical focussing by separately orienting each elastically bent segment. The neutrons diffracted from the specimen are collected in a seven-detector array of ORDELA position-sensitive proportional counters. The detectors are mounted on a circle in the vertical plane and each detector is separated symmetrically above and below the horizontal scattering plane by 7 degrees. The principles of residual stress measurement with neutron diffraction can be found in Ref. [1]. In the present experiment, the (331) reflection from the silicon monochromator at $84^{\circ}$ produced a nominal monochromatic beam of $1.65 \AA$ wavelength. The calibration procedure can be found in Ref. [2]. Five reflections from a nickel powder specimen were used to determine the wavelength. The linear detector position was calibrated against scattering angle in the following procedure. The detector arm carrying the linear position sensitive detector is moved in equal angular increments so that the $311 \mathrm{Ni}$ is displaced through the range of the detector. The observed linear displacement in the peak position is then calibrated with the scattering angle. Incoherent scattering from a polyethylene plate was used to correct for variations in detector efficiency.

Incident and scattered beam apertures defined the gauge volume. The incident beam aperture opening was $1.5 \mathrm{~mm}$ wide and $10 \mathrm{~mm}$ high for normal and transverse strain measurements, 1.5 $\mathrm{mm}$ wide and $2 \mathrm{~mm}$ high for longitudinal strain measurements, at a distance of $45 \mathrm{~mm}$ from the rotation center of the diffractometer. The scattered beam was received through a tall aperture with a $1.5 \mathrm{~mm}$ width for normal and transverse strain measurements and $2 \mathrm{~mm}$ for longitudinal strain measurements, set at $45 \mathrm{~mm}$ from the diffractometer axis. The incident beam was centered over the diffractometer axis by scanning the diffracted intensity from a centered $1 \mathrm{~mm}$ steel pin.

The 211 Bragg reflection position from ferritic iron was used for strain measurements. The experimental parameters including the scattering geometry are summarized in Table I. Diffraction data used in this experiment were taken from the middle three detectors in the detector array. For each detector, diffraction peaks were fitted to a Gaussian curve on a background represented by a second order polynomial. Strains were calculated from the shift in the peak position relative to an appropriate stress-free standard peak position. The reported strains are the average of the strains calculated from the middle three detectors.

For each detector, the error of strain determination was estimated from the uncertainty of fitted peak position. For the average strain value (over three detectors), the root mean square deviation of strains $\left(\sigma_{\varepsilon}^{r m s}\right)$ measured by each detector, which reflects uncertainty of measurements as well as strain variations from grain to grain, was assigned as the estimated error. Typically $\sigma_{\varepsilon}^{r m s}$ is close in magnitude to the individual $\sigma_{\varepsilon}$ values.

Two steel pillar shaped samples (6 $\mathrm{mm}$ x $6 \mathrm{~mm}$ x $40 \mathrm{~mm}$ and $3 \mathrm{~mm} \times 6 \mathrm{~mm} \times 30 \mathrm{~mm}$ ) cut from a common sample of ferritic steel were used for intermediate scattering angle references in both welded plate and stress-free reference experiments. Scattering intensities from one or the other of these samples were recorded periodically throughout the experiment to check the stability and repeatability of peak angle measurement. 
The sample positions were determined by the surface scanning technique [3-4]. Since the surfaces of the plate are not simply related to the directions of specimen translation, a program was devised to convert specimen test point coordinates to translator motor positions [5]. A selection of surface points was located in terms of motor positions by surface scans. A coordinate conversion matrix was calculated using the specimen and motor coordinates. This coordinate conversion was then used to calculate the motor positions to be used in the control program to reach the desired test points. This was particularly useful in measuring points in the stress-free "comb" where the "teeth" in the comb were not generally perpendicular to the plate surface.

\section{RESULTS AND DISCUSSIONS}

The experimental results for the measurements in the weld plate and comb-like sample are shown in Table II. The derived strains and stresses are listed in Table III. The measurement results for the comb-like specimen are plotted in Fig. 2. It can be seen that the strain-free lattice parameter at points close to the front surface varies with distance from the front surface. The variation is due to small composition gradients. The average of the peak position from the measurements at line 1 and 7 in Fig. 2 indicates that the peak shift due to computation gradient is not large (0.03 degrees). The residual strains and stresses at the mid-thickness line are plotted in Fig. 3 and Fig. 4, respectively. Note that the longitudinal stress is tensile and the transverse stresses compressive at the mid-thickness line in the vicinity of the weld. At one end ( $60 \mathrm{~mm}$ from reference line), the residual stresses in all directions tend to be zero while at the other end they are tensile. In all cases the normal stresses are relatively small. The distribution of the residual stresses at the mid-thickness line is not symmetric with respect to the centerline. The residual strain and stress maps at locations on the lines in the vicinity of the weld (line 2 - line 6 ) are shown in Fig. 5 - Fig. 10.

The distribution of scattering intensity from a $0.5 \mathrm{~mm}$ diameter pin that was scanned over a 3 $\mathrm{mm}$ by $2 \mathrm{~mm}$ area on the horizontal plane is shown in Figure [11-17]. The figure indicates good correspondence to the dimensions of the defining slits. The centering of the gauge volume to the diffractometer rotation axis is shown as well. The scanning measurements for the gauge volume indicate that the gauge volume was elongated along the incident beam. The comparison of the gauge volume size for individual detectors is listed in Table IV. The gauge volume size is calculated by measuring the size of the contour in which the intensity is larger than $50 \%$.

\section{CONCLUSIONS}

- The strain and stress distribution at the mid-thickness line is not symmetric with respect to the centerline.

- The lattice parameter of the d-zero sample in the area near the front surface varies due to chemical compositional gradients.

- Compared with the measurement for the ring \& plug, the residual stress measurement for this round robin specimen is more challenging in terms of data interpretation and accurate sample location. 


\section{ACKNOWLEDGEMENT}

Research sponsored by the Assistant Secretary for Energy Efficiency and Renewable Energy, Office of Transportation Technologies, as part of the High Temperature Materials Laboratory User Program, Oak Ridge National Laboratory, managed by Lockheed Martin Energy Research Corp. for the U.S. Department of Energy under contract number DE-AC05-96OR22464. DQW was supported in part by an appointment to the Oak Ridge National Laboratory Postdoctoral Research Associates Program administrated jointly by the Oak Ridge National Laboratory and the Oak Ridge Institute for Science and Education.

\section{REFERENCES}

1. A. Allen, C. Andreani, M. T. Hutchings, and C. G. Windsor, "Measurement of Internal Stress Within Bulk Materials Using Neutron Diffraction", NTD International (October), 249254(1981)

2. X. Zhu and C. R. Hubbard, "A Calibration Procedure for Position Sensitive Detectors Used in Neutron Residual Strain Mapping", Advances in X-ray Analysis, 40, 604-611, (1997)

3. P. C. Brand and H. J. Prask, "New Methods for the Alignment of Instrumentation for Residual Stress Measurements by Means of Neutron Diffraction", J. App. Cryst., 27, 164-176 (1994)

4. D. . Wang and L.Edward, "Precise determination of specimen surface position during subs: Residual Stresses, p135-143, France, 1996

5. D.-Q. Wang, C.R. Hubbard and S. Spooner, "Application of 2-Dimensional coordinate system conversion in stress measurements with neutron diffraction", HTML report, ORNL 1999 


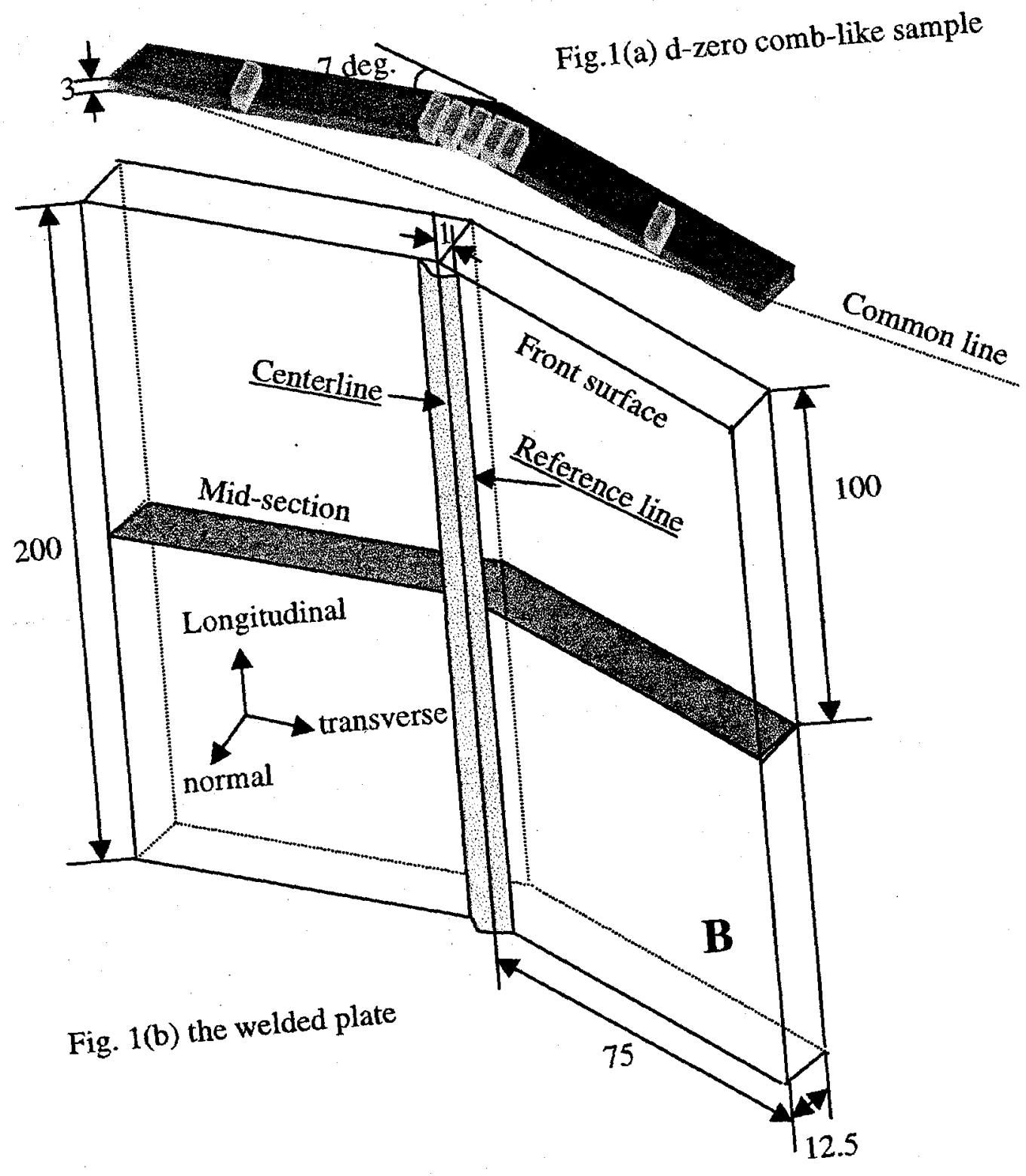

Fig. 1 Schematic drawing of the plate (not to scale). All units are in $\mathrm{mm}$. 


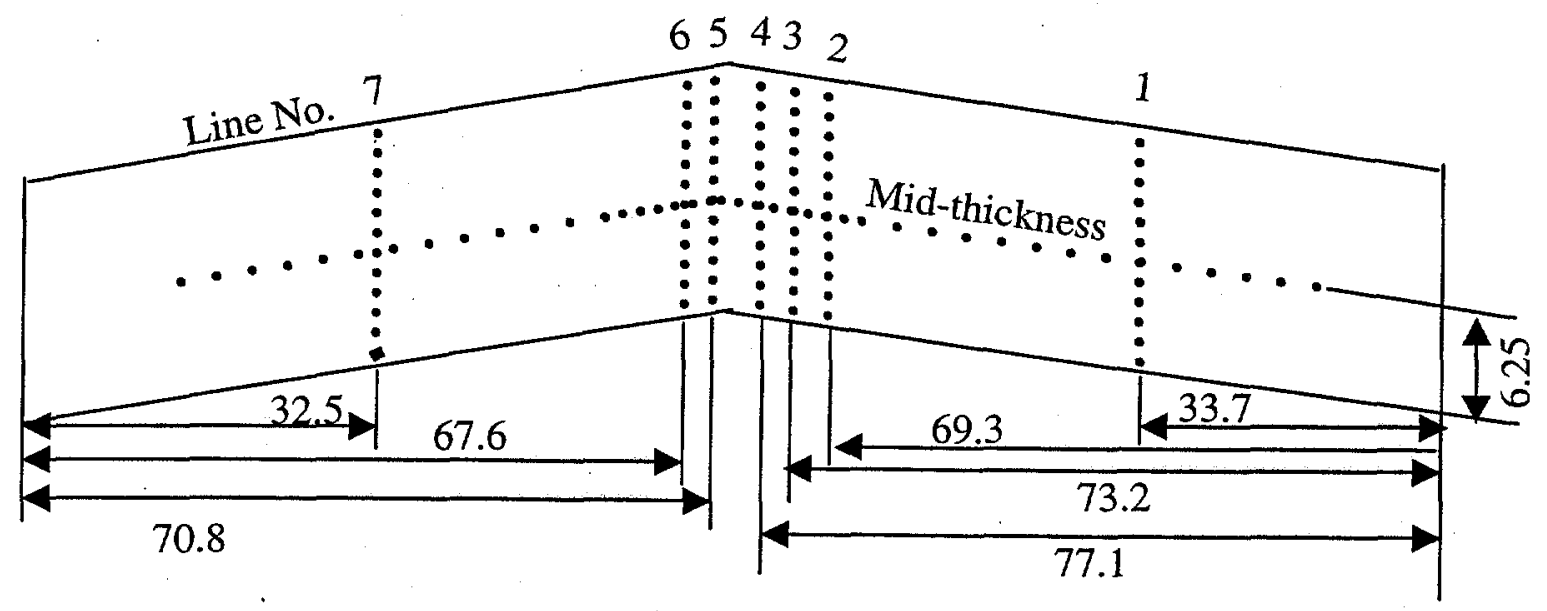

Fig. 1(c) Measurement locations in the mid-section of the plate 
Fig.2 Measured two-theta variation within the comb-like sample at the lines in the vicinity of the weld

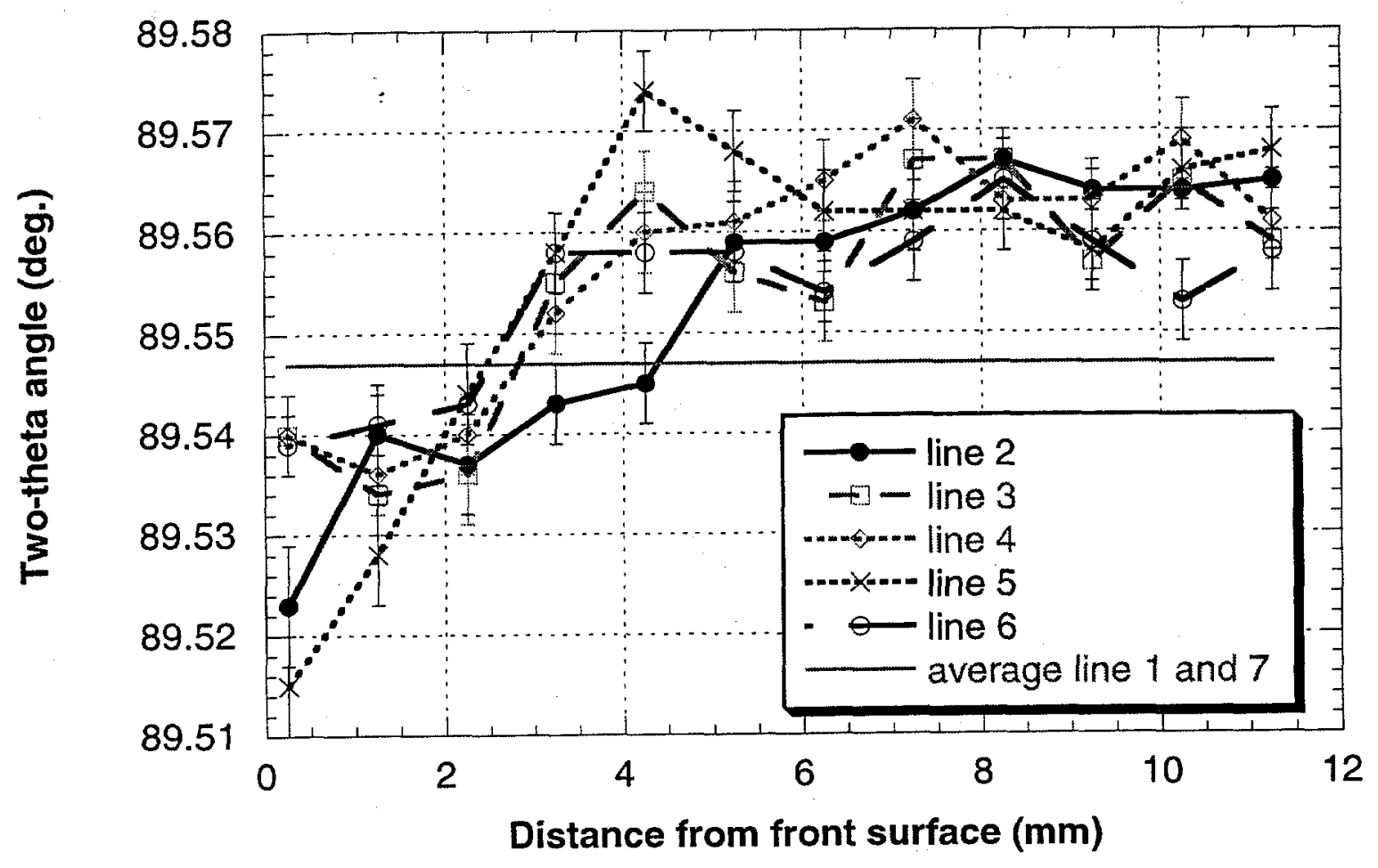


Fig. 3 Residual Strain Distribution at the mid-thickness line

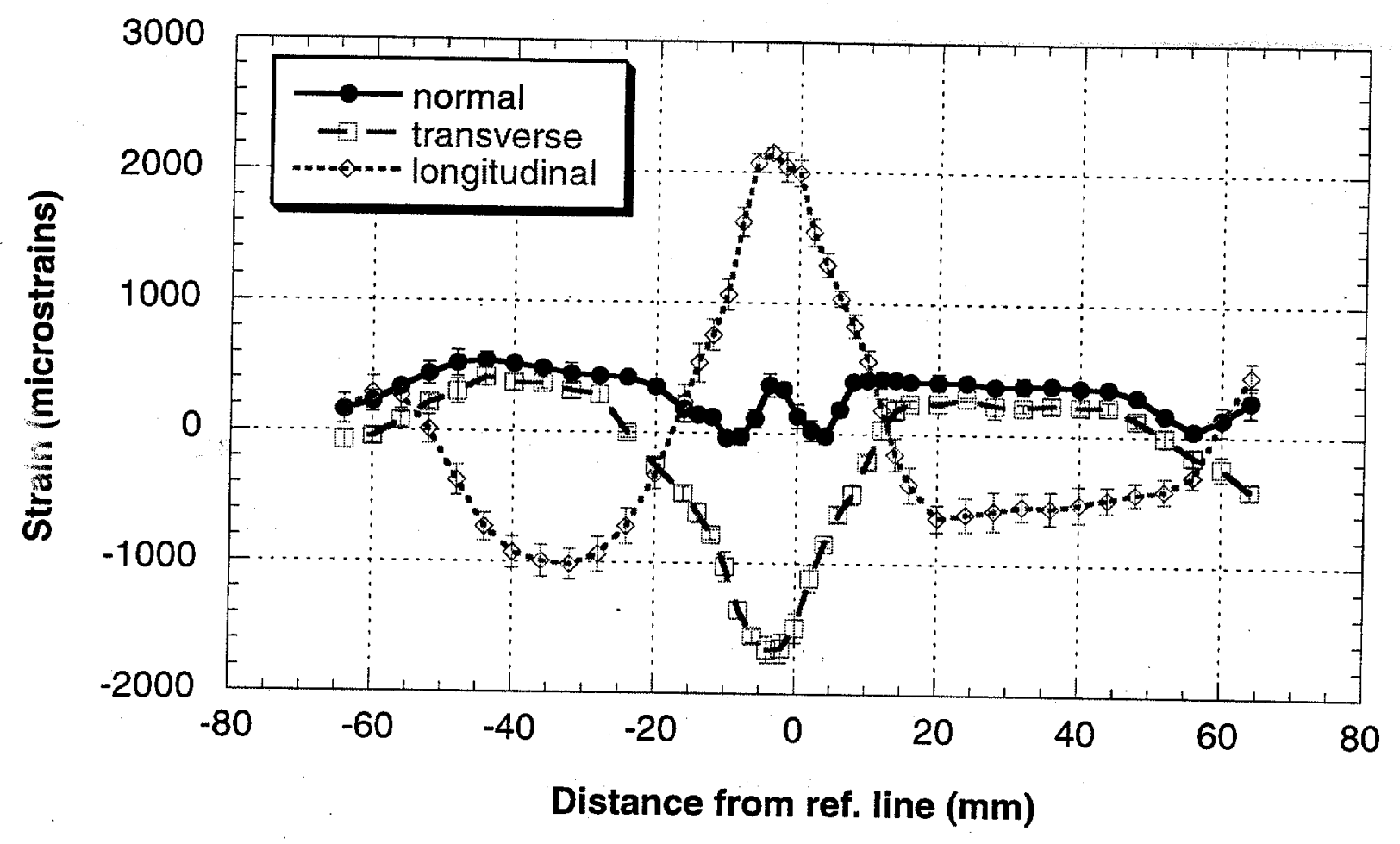


Fig. 4 Stress Distribution at the Mid-thickness Line

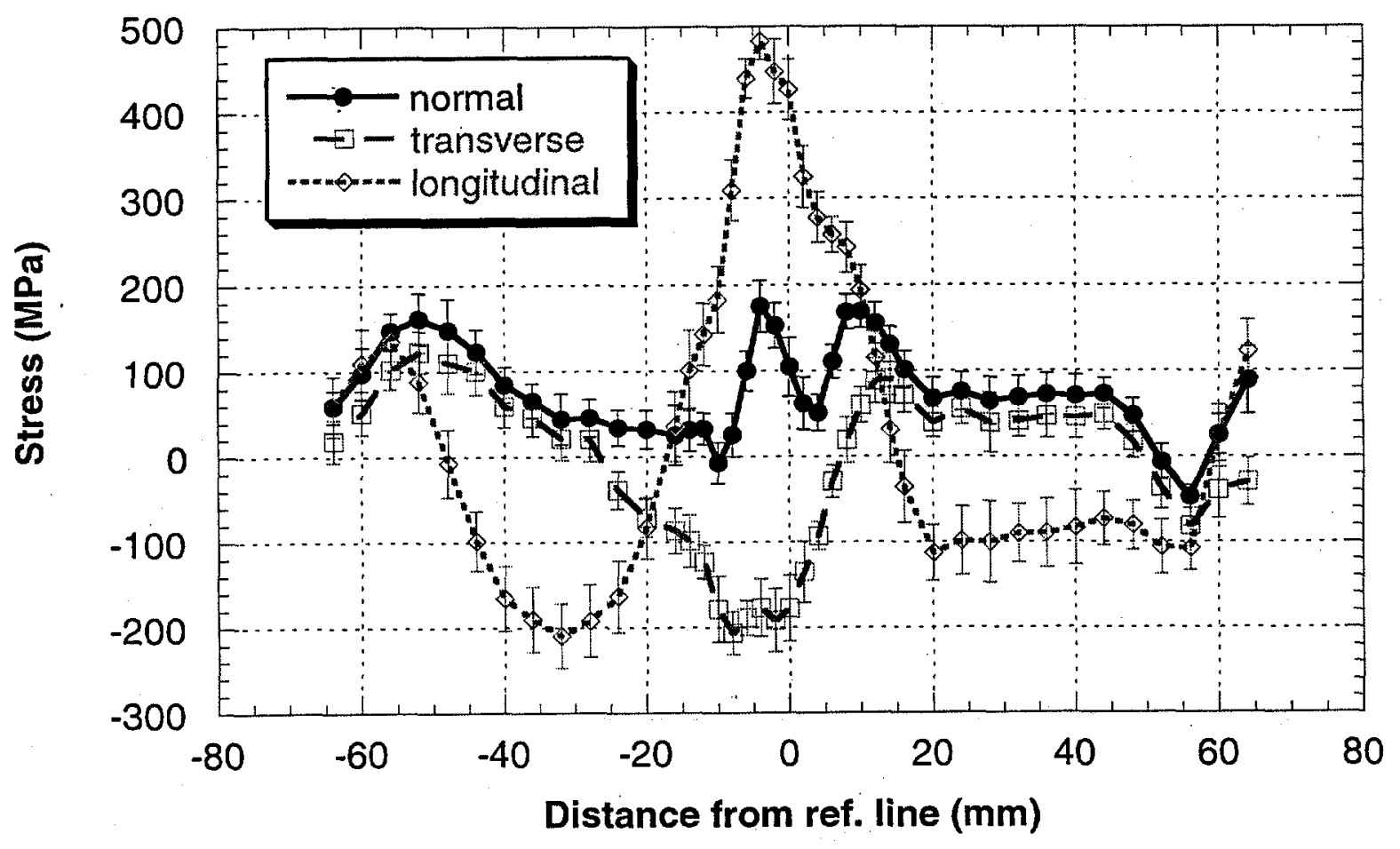




\section{Fig. 6 Normal Stress Distribution in the Vicinity of the Weld}

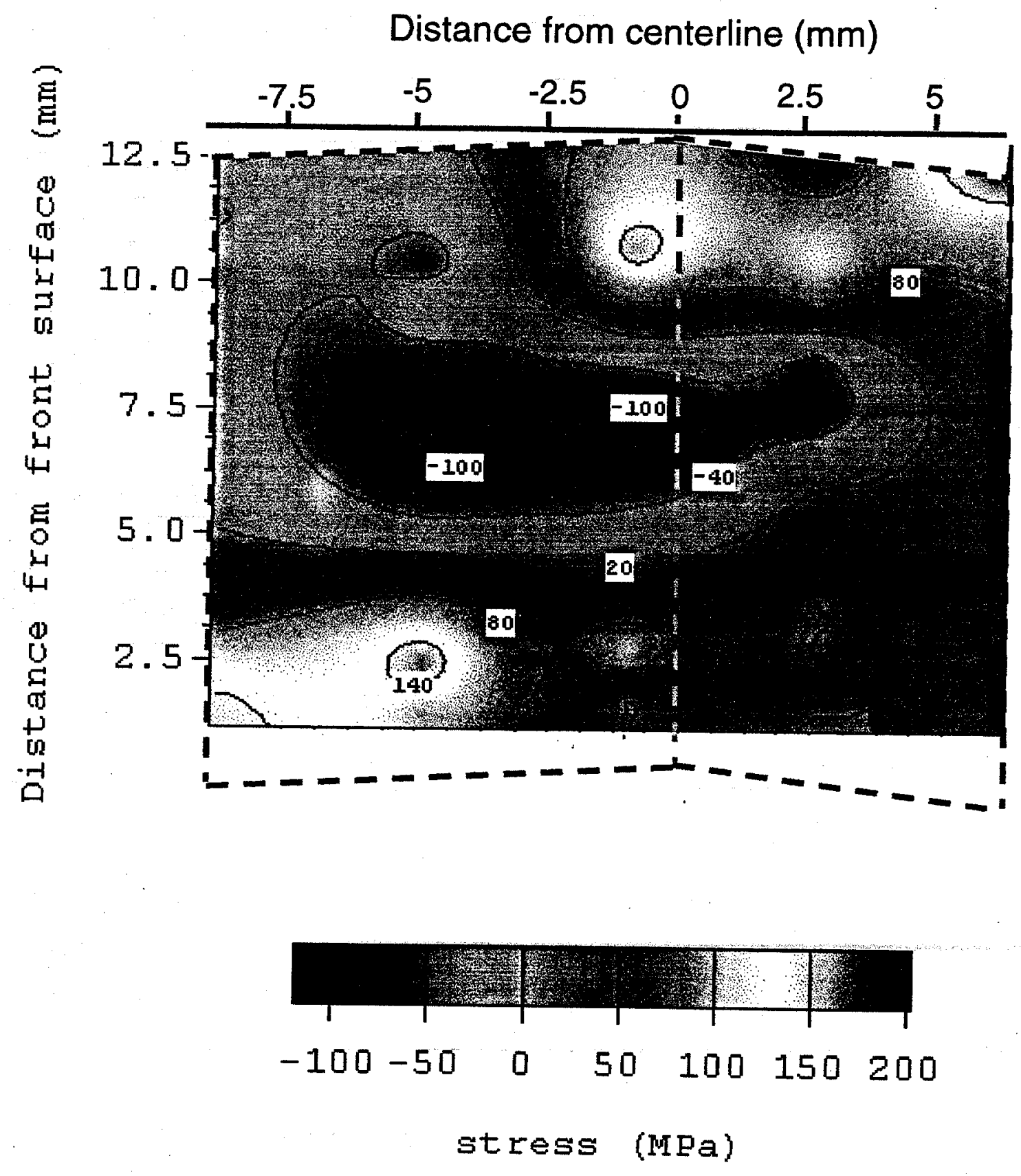




\section{Fig. 7 Transverse Strain Distribution in the Vicinity of the Weld}
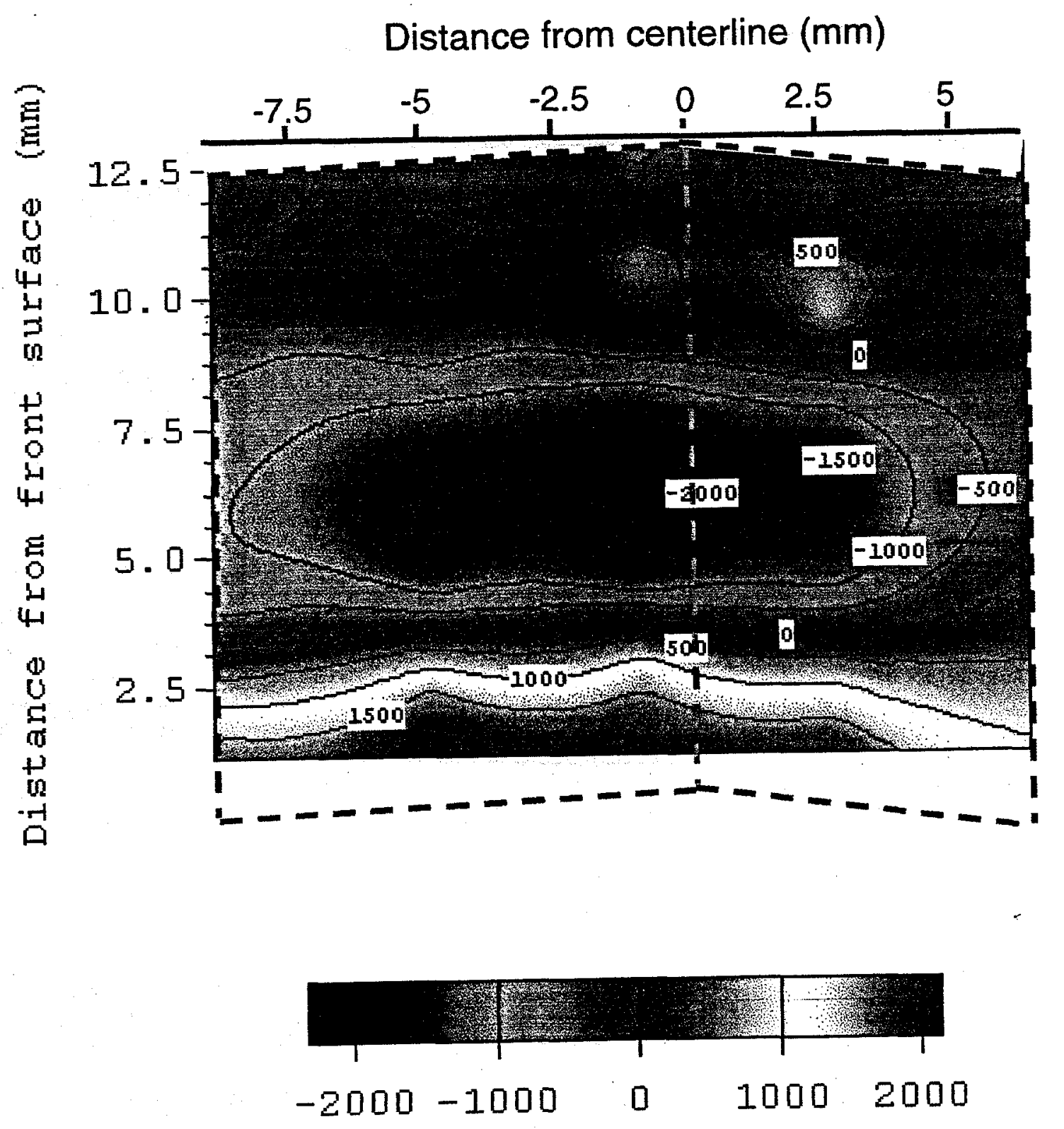

strain (microstrains) 


\section{Fig. 8 Transverse Stress Distribution in the Vicinity of the Weld}
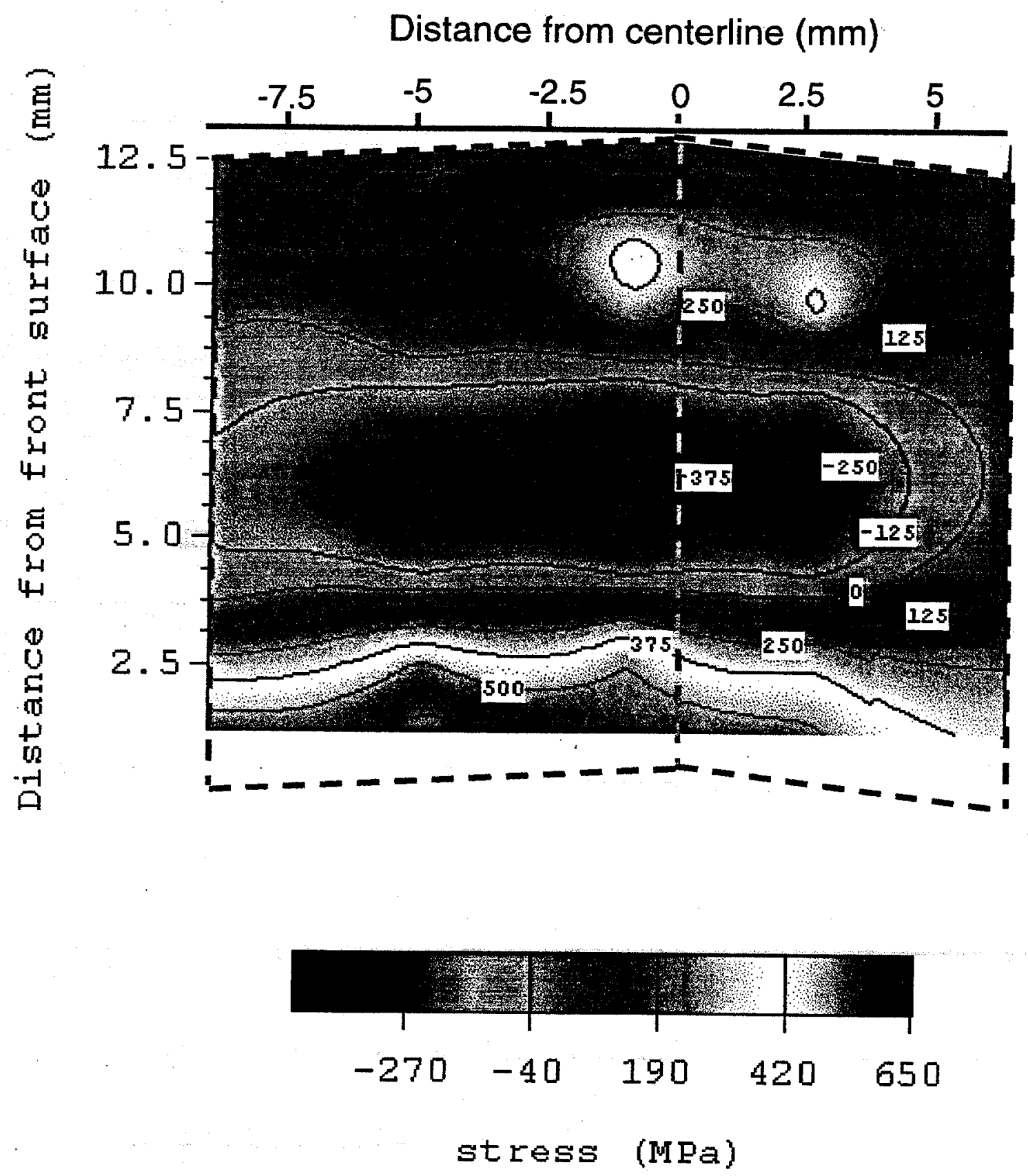


\section{Fig. 9 Longitudinal Strain Distribution in the Vicinity of the Weld}
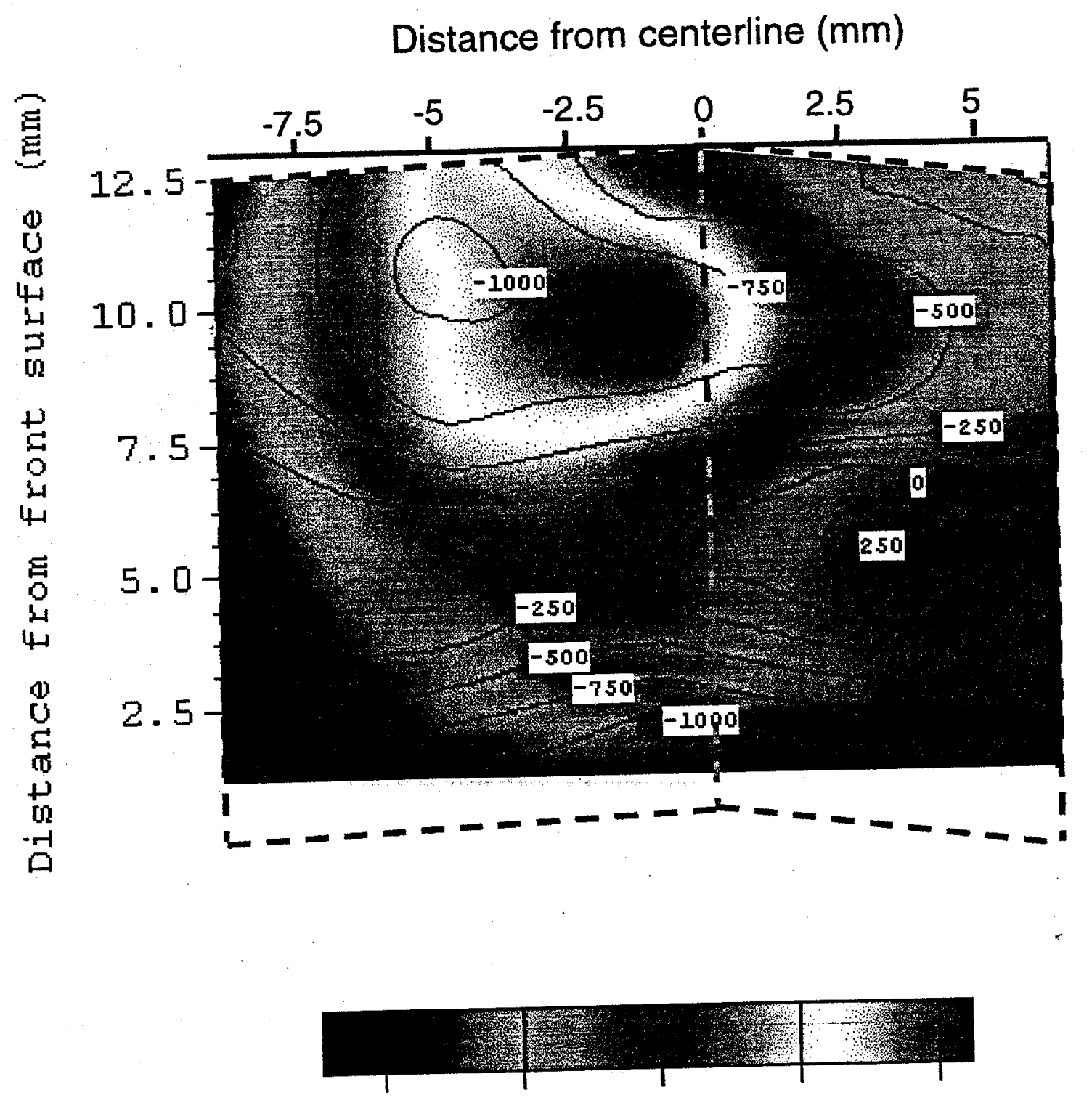

$1000 \quad 1375 \quad 1750 \quad 2125 \quad 2500$

strain (microstrains) 


\section{Fig. 10 Longitudinal Stress Distribution in the Vicinity of the Weld}

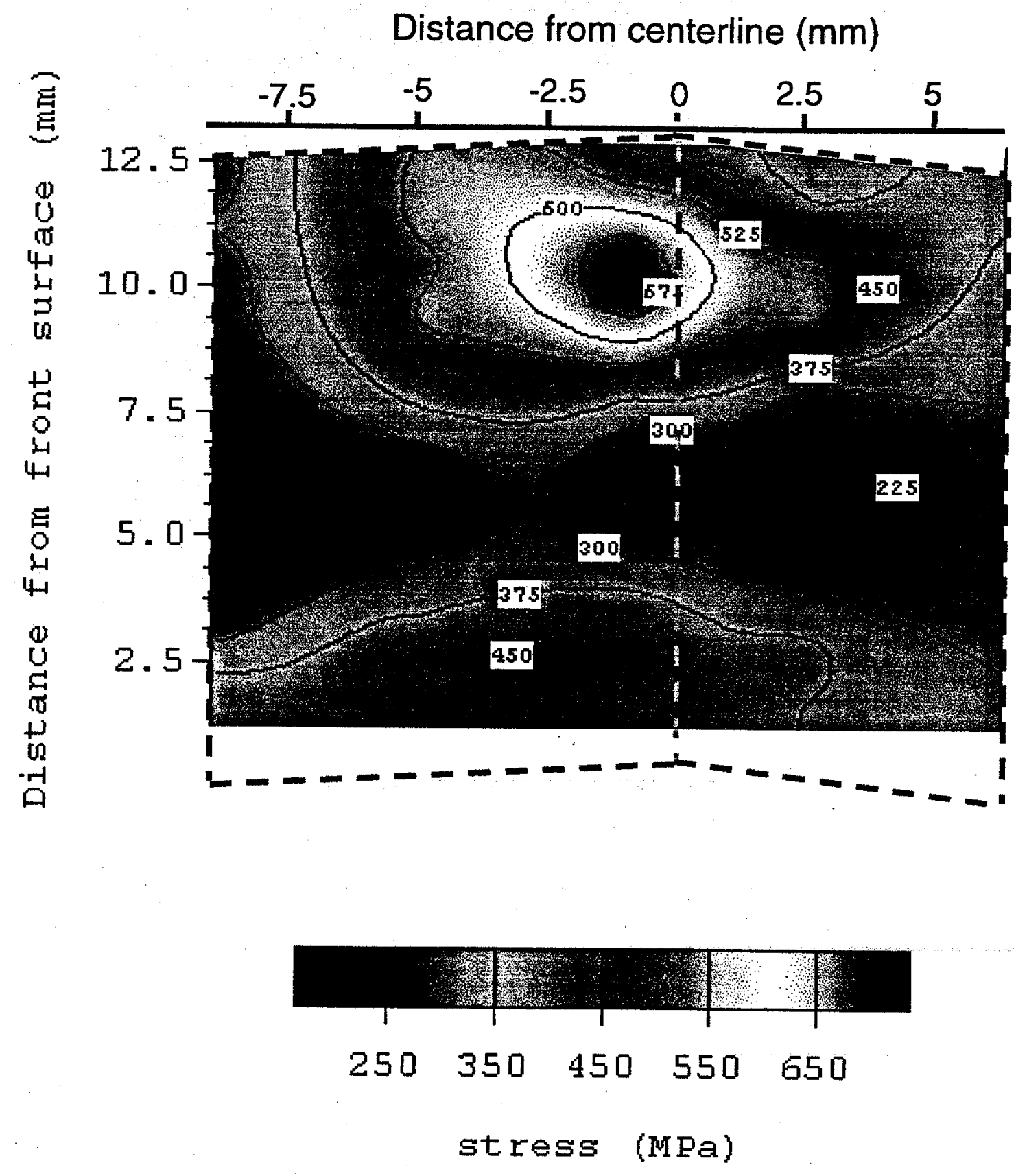


Fig. 11 Gauge volume of detector \#1
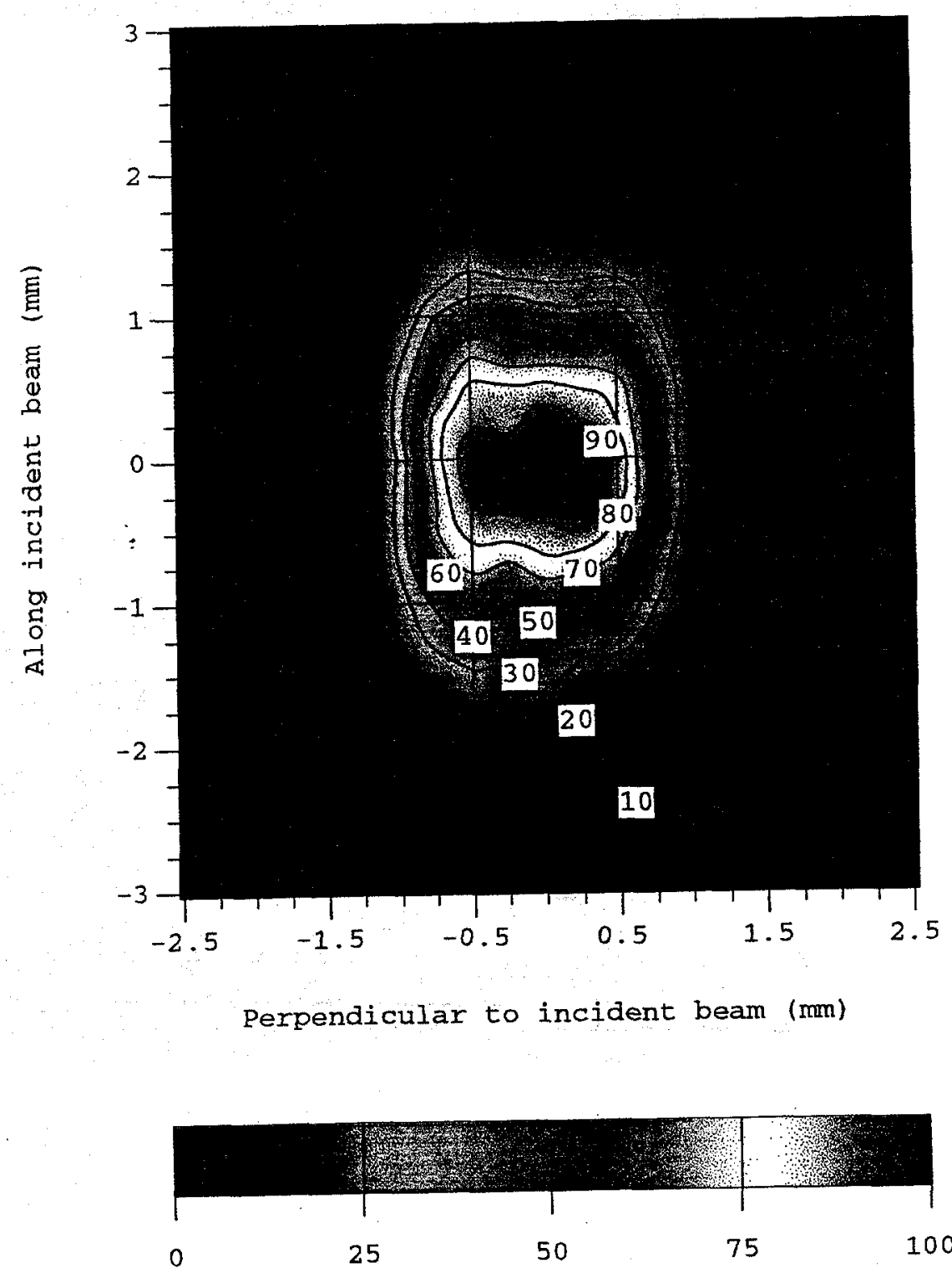

percentage $(8)$ 
Fig. 12 Gauge volume of detector \#2
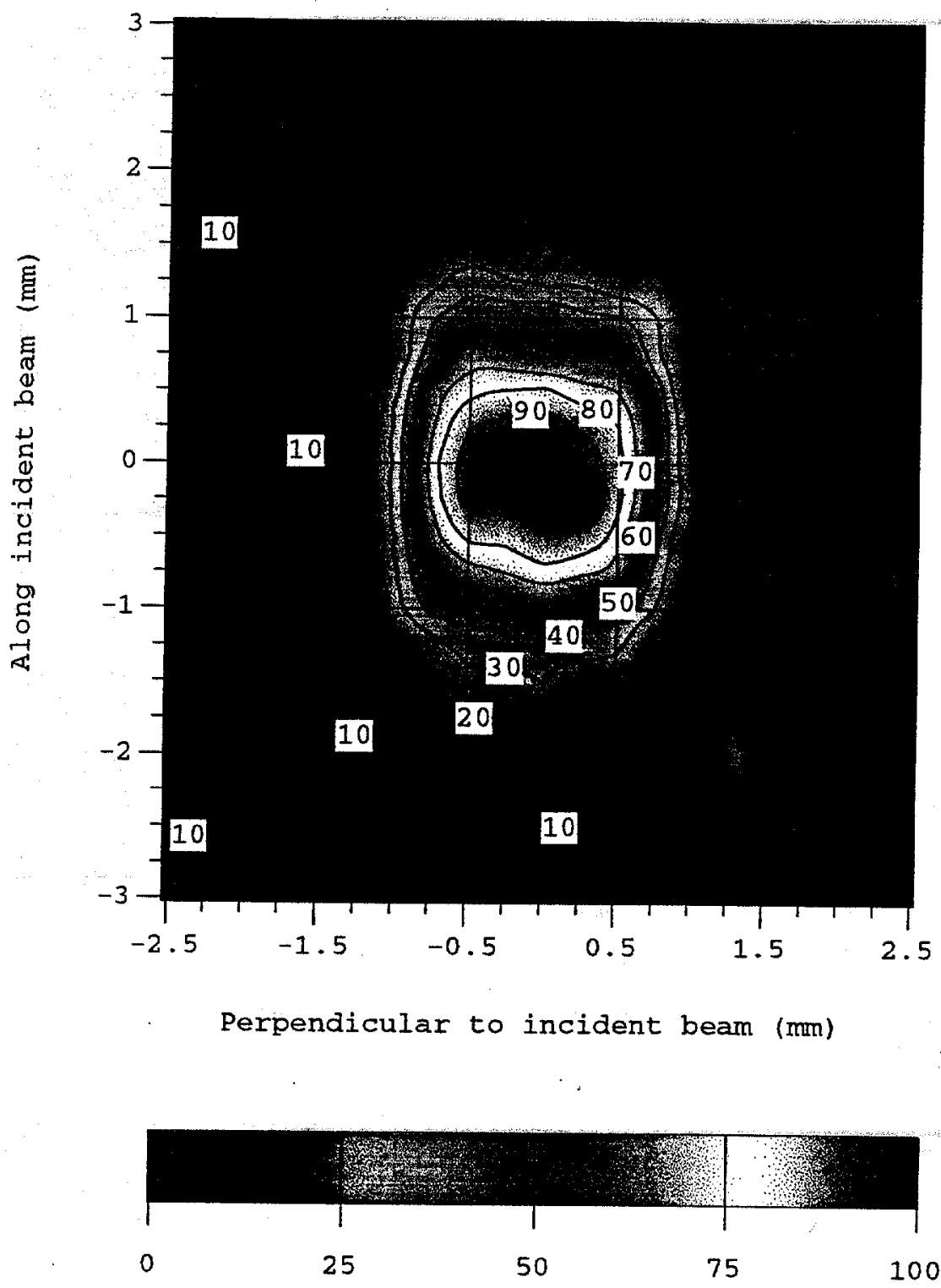

percentage $(8)$ 
Fig. 13 Gauge volume of detector \#3

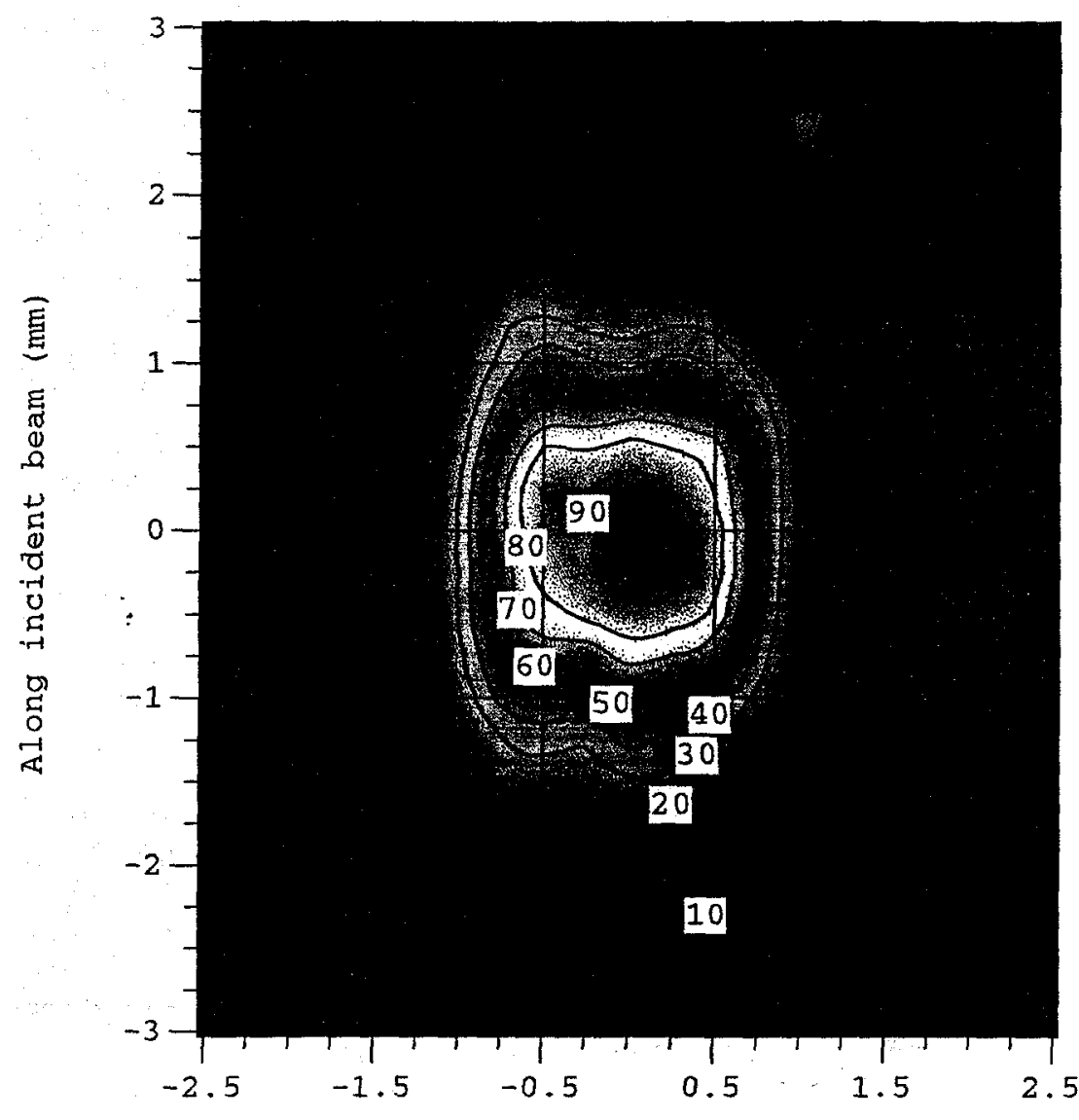

Perpendicular to incident beam (mm)

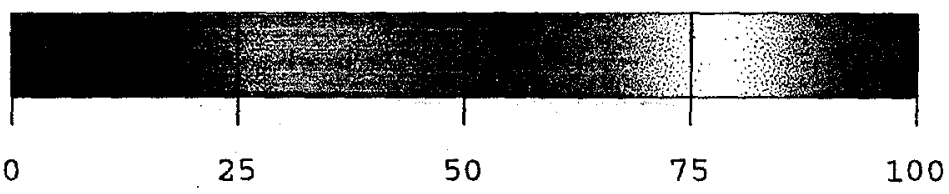

percentage ( $(8)$ 


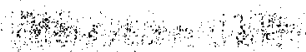

Fig. 14 Gauge volume of detector \#4
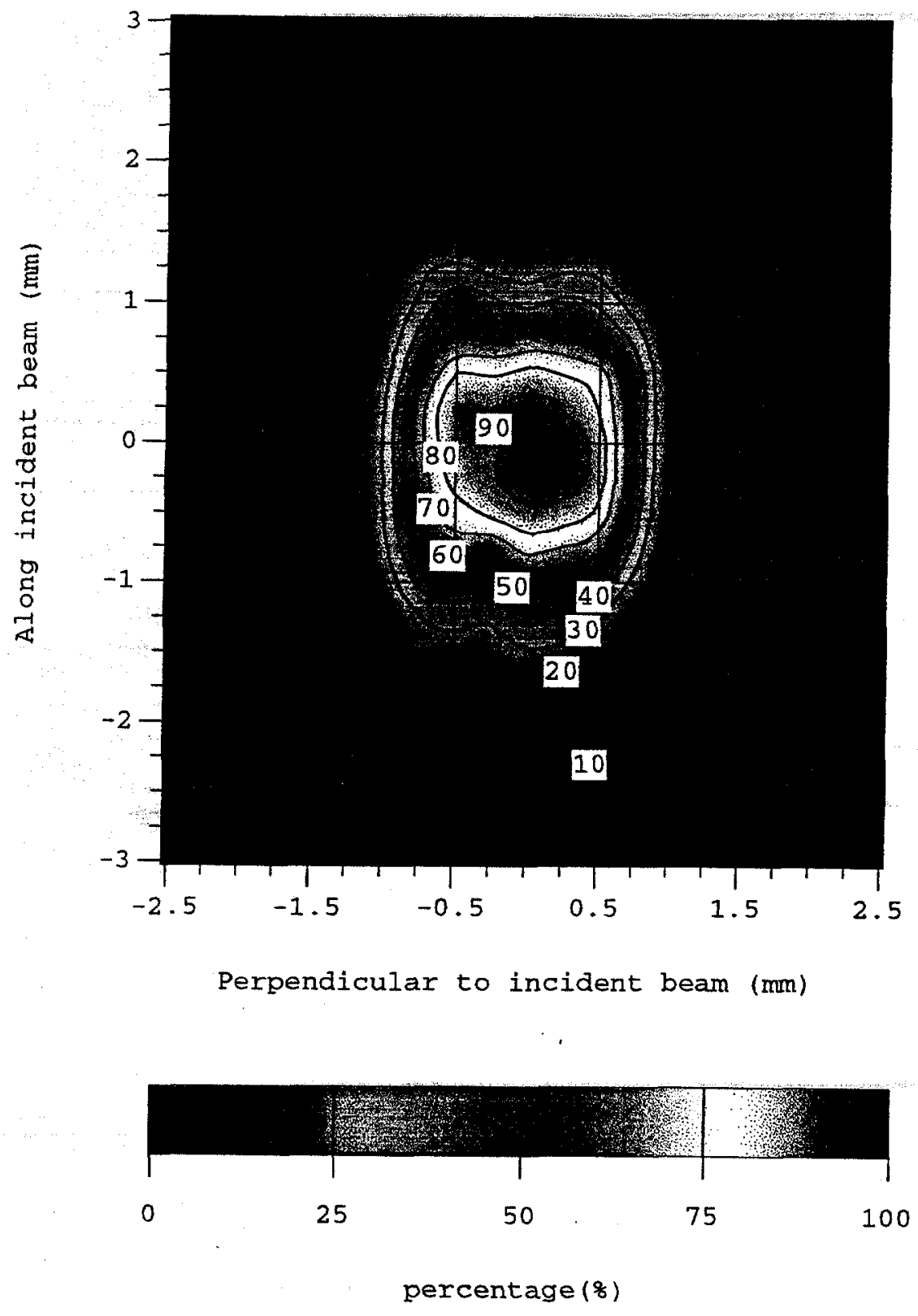
Fig. 15 Gauge volume of detector \#5
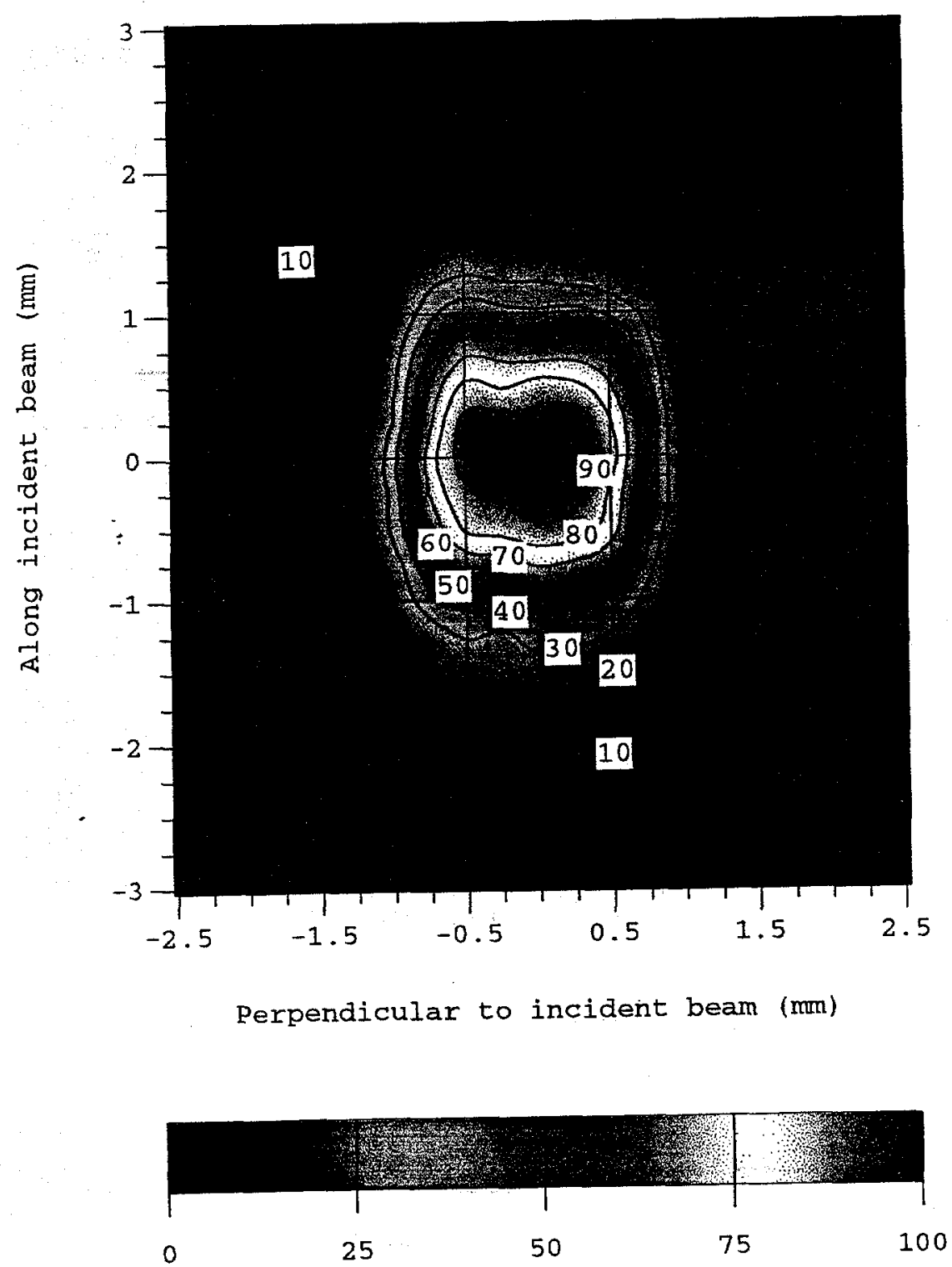

percentage $(8)$ 
Fig. 16 Gauge volume of detector \#6
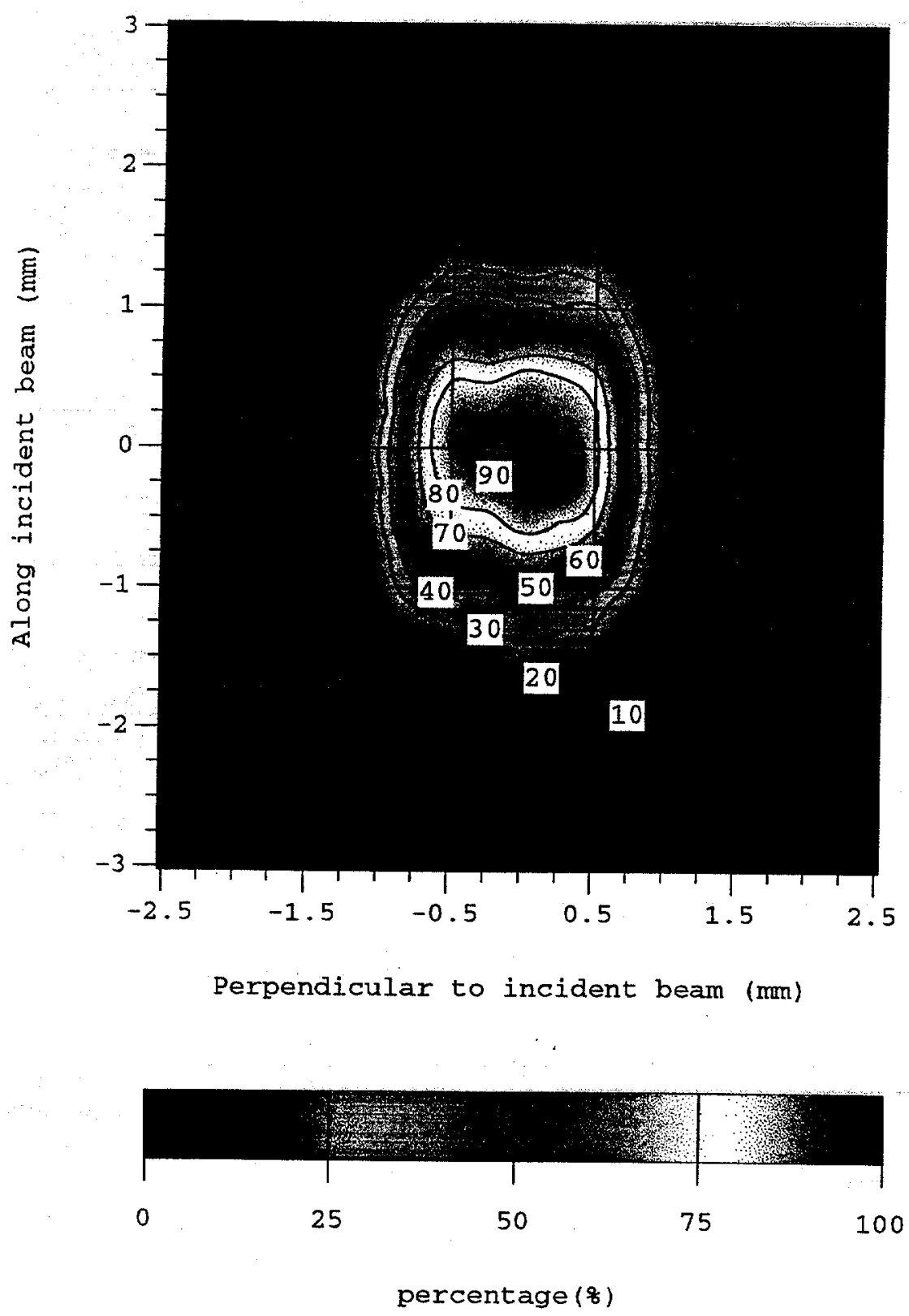
Fig. 17 Gauge volume of detector \#7
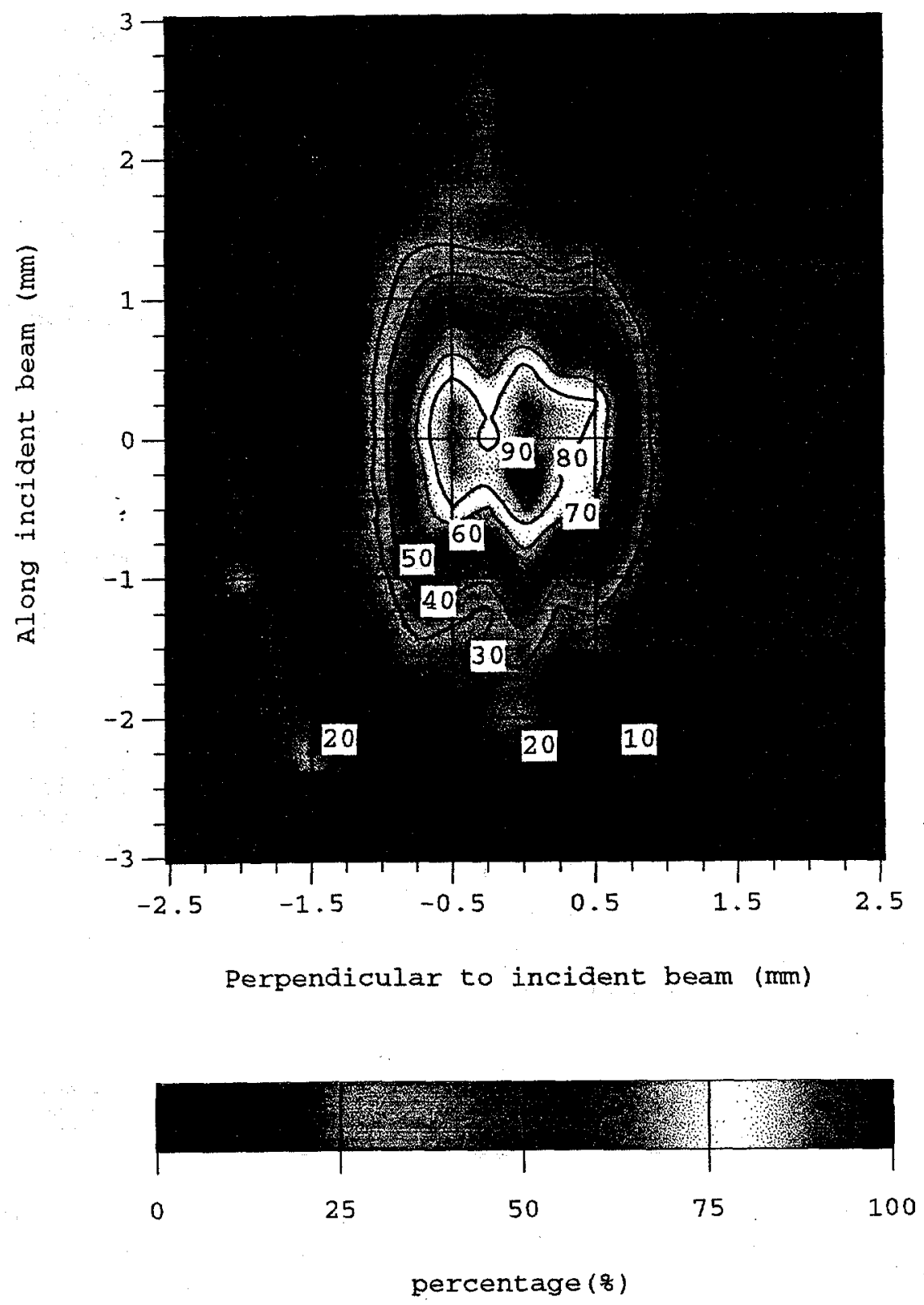
Table I Experimental Parameters Used in the VAMAS TWA-20 Experiment

Double focusing monochromator [Si (331) reflection]

Take-off-angle

$84^{\circ}$

Neutron wavelength

$1.65 \AA$

Sample [Ferritic steel (211) reflection]

Diffraction angle

$\sim 89^{\circ}$

Lattice spacing

$\sim 1.166 \AA$

Geometric parameters

Neutron source-to-monochromator

Monochromator-to-diffractometer axis

$7150 \mathrm{~mm}$

Detector-to-diffractometer axis

$1750 \mathrm{~mm}$

Divergence of in-pile collimator

$800 \mathrm{~mm}$

$0.09^{\circ}$

Incident beam slit-to-diffractometer axis

for normal and transverse strain measurement

$50 \mathrm{~mm}$

for longitudinal strain measurement

$100 \mathrm{~mm}$

Diffracted beam slit-to-diffractometer axis

for normal and transverse strain measurement

for longitudinal strain measurement

$35 \mathrm{~mm}$

$45 \mathrm{~mm}$

Incident slit height for normal and transverse strain measurements

$10 \mathrm{~mm}$

Incident slit height for longitudinal strain measurements

$1.5 \mathrm{~mm}$

Incident slit width for normal and transverse strain measurements

$1.5 \mathrm{~mm}$

Incident slit width for longitudinal strain measurements

Detector slit height

$2 \mathrm{~mm}$

Sampling volume for normal and transverse strain measurements

Sampling volume for longitudinal strain measurements.

$25 \mathrm{~mm}$

$\sim 22.5 \mathrm{~mm}^{3}$

$\sim 6 \mathrm{~mm}^{3}$ 

Table II. Experimental results

\section{Detector \#3}

\begin{tabular}{|c|c|c|c|c|c|c|c|c|}
\hline direction & $\begin{array}{c}\text { Position } \\
(\mathrm{mm})\end{array}$ & $\begin{array}{c}2 \Theta / d / a \\
{[0 / A]}\end{array}$ & uncertainty & $\begin{array}{c}\text { FWHM } \\
{\left[{ }^{\circ}\right]}\end{array}$ & $\begin{array}{c}\text { uncertainty } \\
{\left[{ }^{\circ}\right]}\end{array}$ & $\begin{array}{c}\text { Peak } \\
\text { Height } \\
\end{array}$ & uncertainty & $\begin{array}{c}\text { sampling } \\
\text { time (min.) }\end{array}$ \\
\hline normal & 0.25 & 89.514 & 0.006 & 0.361 & 0.015 & 92.453 & 372 & 18 \\
\hline normal & 1.25 & 89.538 & 0.005 & 0.384 & 0.011 & 135.37 & 3.96 & 15 \\
\hline normal & 2.25 & 89.541 & 0.004 & 0.35 & 0.01 & 120.01 & 3.767 & 10 \\
\hline normal & 3.25 & 89.532 & 0.004 & 0.362 & 0.008 & 151.36 & 4.008 & 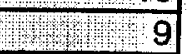 \\
\hline normal & 4.25 & 89.538 & 0.004 & 0.351 & 0.008 & 147.58 & 3.915 & $854 \div 6$ \\
\hline normal & 5.25 & 89.552 & 0.004 & 0.35 & 0.009 & 100.37 & 3.161 & 3 \\
\hline normal & 6.25 & 89.543 & 0.003 & 0.357 & 0.006 & 197.9 & 4.293 & 4 \\
\hline normal & 7.25 & 89.542 & 0.002 & 0.366 & 0.004 & 401.77 & 5.992 & 6 \\
\hline normal & 8.25 & 89.54 & 0.001 & 0.367 & 0.003 & 793.13 & 8.362 & 9 \\
\hline normal & 9.25 & 89.539 & 0.001 & 0.357 & 0.002 & 1220.8 & 10.432 & 10 \\
\hline normal & 10.25 & 89.538 & 0.001 & 0.364 & 0.002 & 2397.2 & 14.348 & 15 \\
\hline normal & 11.25 & 89.536 & 0.001 & 0.359 & 0.001 & 3762.5 & 18.025 & 18 \\
\hline normal & 0.25 & 89.606 & 0.006 & 0.377 & 0.014 & 93.696 & 3.613 & 18 \\
\hline normal & 1.25 & 89.615 & 0.005 & 0.39 & 0.01 & 137.36 & 3.958 & 15 \\
\hline normal & 2.25 & 89.612 & 0.004 & 0.383 & 0.01 & 126.98 & 3.703 & 10 \\
\hline normal & 3.25 & 89.606 & 0.004 & 0.373 & 0.008 & 164.13 & 4.09 & 9 \\
\hline normal & 4.25 & 89.599 & 0.004 & 0.374 & 0.008 & 146.31 & 3.806 & 6 \\
\hline normal & 5.25 & 89.588 & 0.004 & 0.396 & 0.009 & 96.943 & 43.11 & 3 \\
\hline normal & 6.25 & 89.564 & 0.003 & 0.387 & 0.007 & 183.14 & 175.157 & 4 \\
\hline normal & 7.25 & 89.564 & 0.002 & 0.378 & 0.004 & 390.42 & 5.843 & 6 \\
\hline normal & 8.25 & 89.563 & 0.001 & 0.378 & 0.003 & 759.66 & 8.111 & 9 \\
\hline normal & 9.25 & 89.579 & 0.001 & 0.373 & 0.002 & 1171 & 10.028 & 10 \\
\hline normal & 10.25 & 89.603 & 0.001 & 0.373 & 0.002 & 2329.5 & 14.026 & 15 \\
\hline normal & 17.25 & 89.63 & 0.001 & 0.374 & 0.001 & 3711.2 & 17.59 & 18 \\
\hline normal & 0.25 & 89.662 & 0.009 & 0.502 & 0.021 & .73 .228 & 2.97 & 18 \\
\hline normal & 1.25 & 89.673 & 0.007 & 0.583 & 0.017 & 98.531 & 2.922 & 15 \\
\hline normal & 2.25 & 89.682 & 0.008 & 0.643 & 0.019 & 79.799 & 2.42 & 10 \\
\hline normal & 3.25 & 89.673 & 0.005 & 0.496 & 0.012 & 120.1 & 3.191 & 9 \\
\hline normal & 4.25 & 89.657 & 0.004 & 0.425 & 0.01 & 130.55 & 3.396 & की \\
\hline normal & 5.25 & 89.637 & 0.005 & 0.453 & 0.012 & 85.319 & 2.611 & 3 \\
\hline normal & 6.25 & 89.599 & 0.004 & 0.445 & 0.008 & 159.76 & 3.569 & -4 \\
\hline normal & 7.25 & 89.572 & 0.002 & 0.438 & 0.005 & 342.36 & 5.172 & 6 \\
\hline normal & 8.25 & 89.574 & 0.002 & 0.42 & 0.003 & 694.99 & 7.402 & 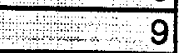 \\
\hline normal & 9.25 & 89.589 & 0.001 & 0.409 & 0.003 & 1070.2 & 9.245 & 10 \\
\hline normal & 10.25 & 89.633 & 0.001 & 0.406 & 0.002 & 2136.4 & 12.947 & 15 \\
\hline normal & 11.25 & 89.666 & 0.001 & 0.398 & 0.001 & 3405.7 & 16.457 & 18 \\
\hline normal & 0.25 & 89.575 & & 0.651 & 0.022 & 85.39 & 2.744 & 18 \\
\hline normal & 1.25 & 89.596 & 0.008 & 0.651 & 0.018 & 98.549 & 2.775 & 15 \\
\hline normal & 2.25 & 89.644 & 0.006 & 0.512 & 0.013 & 106.08 & 3 & 10 \\
\hline normal & 3.25 & 89.665 & 0.004 & 0.429 & 0.009 & 156.24 & 3.767 & 9 \\
\hline normal & \begin{tabular}{|l|}
4.25 \\
\end{tabular} & 89.649 & 0.004 & 0.447 & 0.009 & 143.17 & 3.47 & 6 \\
\hline normal & 5.25 & 89.623 & 0.005 & 0.441 & 0.011 & 89.138 & 2.723 & 3 \\
\hline normal & 6.25 & 89.598 & 0.004 & 0.457 & 0.008 & 152.62 & 3.434 & 4 \\
\hline normal & 7.25 & 89.552 & 0.003 & 0.499 & 0.006 & 297.41 & 4.545 & 6 \\
\hline
\end{tabular}




\begin{tabular}{|c|c|c|c|c|c|c|c|c|}
\hline normal & 8.25 & 89.555 & 0.002 & 0.504 & 0.004 & 566.487 & 6.199 & 9 \\
\hline normal & 9.25 & 89.604 & 0.002 & 0.473 & 0.003 & 904.359 & 7.968 & 10 \\
\hline normal & 10.25 & 89.654 & 0.001 & 0.431 & 0.002 & 2017.597 & 12.298 & 15 \\
\hline normal & 17.25 & 89.685 & 0.001 & 0.426 & 0.002 & 3128.601 & 15.258 & 18 \\
\hline normal & 0.25 & 89.568 & 0.01 & 0.67 & 0.023 & 79.223 & 2.626 & 18 \\
\hline normal & 1.25 & 89.589 & 0.008 & 0.671 & 0.02 & 89.656 & 172.658 & 15 \\
\hline normal & 2.25 & 89.619 & 0.007 & 0.598 & 0.018 & 87.905 & 2.652 & 10 \\
\hline normal & 3.25 & 89.66 & 0.005 & 0.471 & 0.01 & 140.528 & 3.456 & 12 9 \\
\hline normal & 4.25 & 89.662 & 0.004 & 0.456 & 140 0.07 & 136.871 & 3.366 & 6 \\
\hline normal & 5.25 & 89.609 & 0.006 & 0.488 & 0.012 & 86.494 & $2 \times 2.56$ & 3 \\
\hline normal & 6.25 & 89.542 & 0.004 & 0.481 & 0.008 & 160.347 & 3.43 & 4 \\
\hline normal & 7.25 & 89.528 & 0.003 & 0.481 & 0.006 & 318.906 & 4769 & 6 \\
\hline normal & 8.25 & 89.532 & 0.002 & 0.478 & 0.004 & 627442 & 6.707 & 9 \\
\hline normal & 9.25 & 89.574 & 0.001 & 0.44 & 0.003 & 999.923 & $8 \% 846$ & 10 \\
\hline normal & 10.25 & 89.631 & 0.001 & 0.422 & 0.002 & 2116.614 & 12.68 & W. \\
\hline normal & 11,25 & 89.672 & 0.001 & 0.417 & 0.001 & 3321,073 & 15.859 & Pli 18 \\
\hline normal & 0.25 & 89.539 & 0.007 & 0.443 & 0.017 & 91.835 & 3.42 & 18 \\
\hline normal & 1.25 & 89.576 & 0.004 & 0.386 & 0.01 & 144.835 & 4.098 & 15 \\
\hline normal & 2.25 & 89.601 & 0.004 & 0.374 & 0.009 & 139.545 & 3.888 & 10 \\
\hline normal & 3.25 & $: 89.634$ & 0.004 & 0.384 & 0.008 & 165.105 & 4.08 & 9 \\
\hline normal & 4.25 & 89.633 & 0.004 & 0.406 & 0.008 & 151.002 & 3.7 & 6 \\
\hline normal & 5.25 & 89.606 & 0.004 & 0.4 & 0.009 & 104.083 & 3.014 & 3 \\
\hline normal & 6.25 & 89.582 & 0.003 & 0.389 & 0.007 & 190.517 & 4.12 & 4 \\
\hline normal & 7.25 & 89.567 & 0.002 & 0.388 & 0.004 & 395.773 & 5.824 & 6 \\
\hline normal & 8.25 & 89.56 & 0.001 & 0.391 & 0.003 & 779.639 & 8.065 & 9 \\
\hline normal & 9.25 & 89.575 & 0.001 & 0.387 & 0.002 & 1152.7 & 9.773 & 10 \\
\hline normal & 10.25 & 89.61 & 0.001 & 0.388 & 0.002 & 2218.919 & 13.487 & 15 \\
\hline normal & 11.25 & 89.643 & 0.001 & 0.393 & 0.001 & 3456.674 & 16.617 & 18 \\
\hline normal & 0.25 & 89.55 & 0.026 & 0.384 & 0.062 & 5.122 & 0.816 & 1 \\
\hline normal & 1.25 & 89.52 & 0.015 & 0.316 & 0.033 & 9.263 & 1.073 & 1 \\
\hline normal & 2.25 & 89.54 & 0.012 & 0.393 & 0.032 & 20.84 & 1.397 & 1.5 \\
\hline normal & 3.25 & 89.526 & 0.009 & 0.356 & 0.018 & 27.128 & 1.669 & 1.5 \\
\hline normal & 4.25 & 89.539 & 0.006 & 0.354 & 0.013 & 53.398 & 2.301 & 2 \\
\hline normal & 5.25 & 89.545 & 0.004 & 0.369 & 0.009 & 105.912 & 3.133 & 3 \\
\hline normal & 6.25 & 89.543 & 0.003 & 0.364 & 0.006 & 195.021 & 4.232 & 4 \\
\hline normal & 7.25 & 89.546 & 0.002 & 0.355 & 0.004 & 392.683 & 6.038 & 6 \\
\hline normal & 8.25 & 89.54 & 0.001 & 0.36 & 0.003 & 801.217 & 8.469 & 9 \\
\hline normal & 9.25 & 89.542 & 0.001 & 0.358 & 0.002 & 1217.958 & 10.409 & 10 \\
\hline normal & 10.25 & 89.541 & 0.001 & 0.361 & 0.002 & 2418.092 & 14.458 & 15 \\
\hline normal & 11.25 & 89.533 & 0.001 & 0.361 & 0.001 & 3861.869 & 18.227 & 1.8 \\
\hline normal & -64 & 89.537 & 0.003 & 0.359 & 0.006 & 204.218 & 4.377 & 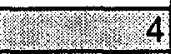 \\
\hline normal & $6 \quad 60$ & 89.533 & 0.003 & 0.359 & 0.006 & 210.908 & 48.446 & 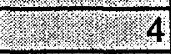 \\
\hline normal & Y 56 & 89.521 & 0,003 & 0.359 & 0.006 & 2071112 & 4.389 & $4 \quad 4$ \\
\hline normal & $\begin{array}{r}752 \\
\end{array}$ & 89.508 & 0.003 & 0.361 & 0.006 & 216.184 & 40.484 & 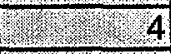 \\
\hline normal & 48 & 89.491 & 0.003 & 0.362 & 0.006 & 208.974 & J 4385 & W7V? 4 \\
\hline normal & 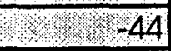 & 89.49 & 0.003 & 0.36 & 0.006 & 211028 & 4429 & $4 \quad 4$ \\
\hline normal & 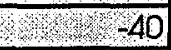 & 89.495 & 0.003 & 0.358 & 0.006 & 214724 & 4473 & 278 \\
\hline normal & 78 36 & 89.502 & 0.003 & 0.356 & 0.006 & 217652 & 4.546 & WVy \\
\hline normal & $7 \% 32$ & 89.497 & 0.003 & 0.352 & 0.006 & 217504 & 4.564 & 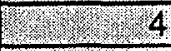 \\
\hline normal & 17\% 28 & 89.508 & 0.003 & 0.37 & 0.006 & $2 / 4766$ & 4.399 & $\begin{array}{r}78 \\
\end{array}$ \\
\hline normal & $4 \quad 24$ & 89.506 & 0.003 & 0.353 & 0.006 & 214.313 & 47.524 & 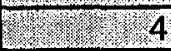 \\
\hline normal & 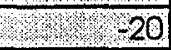 & 89.508 & 0.003 & 0.364 & 0.006 & 214.429 & 104.43 & 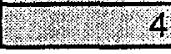 \\
\hline
\end{tabular}




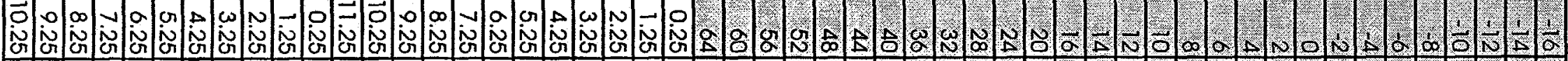

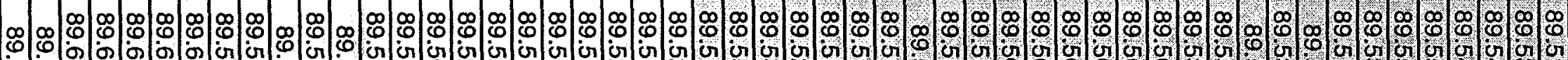
当

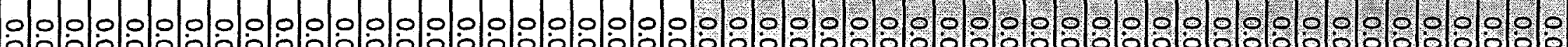

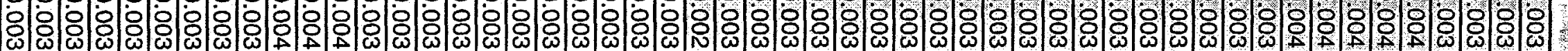

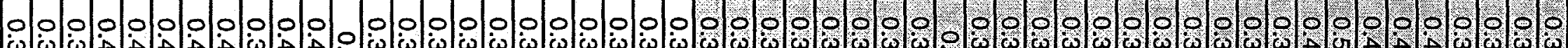
v

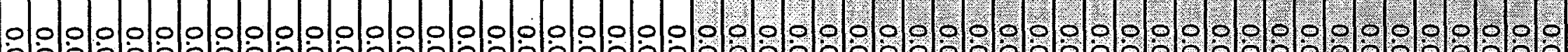

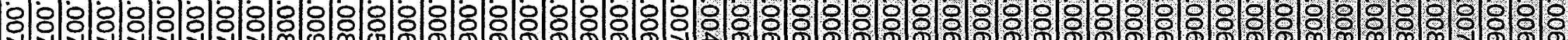

-

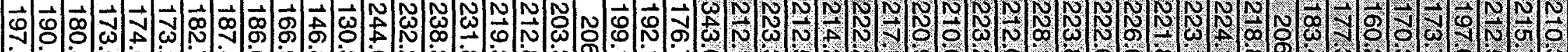

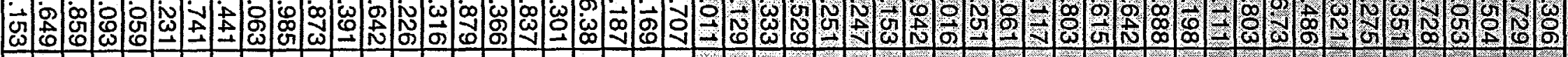

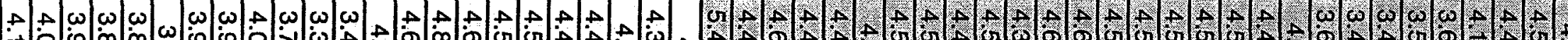

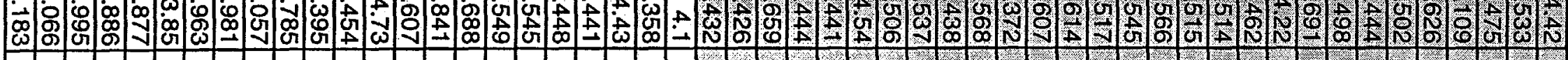

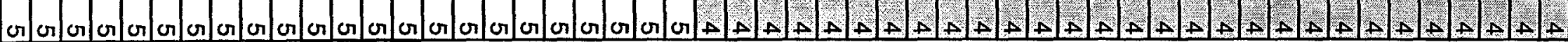




\begin{tabular}{|c|c|c|c|c|c|c|c|c|}
\hline transverse & 11.25 & 89.382 & 0.004 & 0.412 & 0.009 & 133.178 & 3.41 & 5 \\
\hline transverse & 0.25 & 89.58 & 0.004 & 0.533 & 0.009 & 145.775 & 3.133 & 5 \\
\hline transverse & 7.25 & 89.538 & 0.004 & 0.519 & 0.01 & 144.486 & 3.2 & 5 \\
\hline transverse & 2.25 & 89.543 & 0.004 & 0.504 & 0.009 & 143.513 & 3.232 & 5 \\
\hline transverse & 3.25 & 89.566 & 0.004 & 0.442 & 0.008 & 164.348 & 3.642 & 5 \\
\hline transverse & 4.25 & 89.627 & 0.004 & 0.472 & 0.009 & 152.243 & 3.418 & 5 \\
\hline transverse & 5.25 & 89.726 & 0.004 & 0.484 & 0.009 & 147.209 & 3.323 & 5 \\
\hline transverse & 6.25 & 89.791 & 0.004 & 0.484 & 0.009 & 154.103 & 3.389 & 5 \\
\hline transverse & 7.25 & 89.793 & 0.004 & 0.487 & 0.009 & 157.294 & 3.42 & 5 \\
\hline transverse & 8.25 & 89.712 & 0.004 & 0.463 & 0.008 & 170.46 & 3.631 & 5 \\
\hline transverse & 9.25 & 89.545 & 0.004 & 0.446 & 0.008 & 183.242 & 3.806 & 5 \\
\hline transverse & 10.25 & 89.415 & 0.003 & 0.433 & 0.007 & 179.756 & 3.785 & 5 \\
\hline transverse & 11.25 & 89.347 & 0.005 & 0.451 & 0.01 & 116.21 & 3.074 & 5 \\
\hline transverse & 0.25 & 89.612 & 0.006 & 0.649 & 0.013 & 107.792 & 2.512 & 5 \\
\hline transverse & 1.25 & 89.552 & 0.005 & 0.575 & 0.011 & 121.911 & 2.819 & 5 \\
\hline transverse & 2.25 & 89.472 & 0.004 & 0.48 & 0.009 & 150.199 & 3.359 & 5 \\
\hline transverse & 3.25 & 89.497 & 0.004 & 0.493 & 0.009 & 152.068 & 3.314 & 5 \\
\hline transverse & 4.25 & 89.631 & 0.004 & 0.475 & 0.009 & 156.407 & 3.455 & 5 \\
\hline transverse & 5.25 & 89.739 & 0.004 & 0.483 & 0.008 & 166.894 & 3.494 & 5 \\
\hline transverse & 6.25 & 89.816 & 0.004 & 0.47 & 0.008 & 178.587 & 3.643 & 5 \\
\hline transverse & 7.25 & 89.839 & 0.004 & 0.491 & 0.008 & 179.577 & 3.608 & 5 \\
\hline transverse & 8.25 & 89.759 & 0.004 & 0.501 & 0.008 & 180.429 & 3.542 & 5 \\
\hline transverse & 9.25 & 89.555 & 0.004 & 0.484 & 0.008 & 190.702 & 3.708 & 5 \\
\hline transverse & 10.25 & 89.423 & 0.003 & 0.436 & 0.007 & 198.702 & 3.978 & 5 \\
\hline transverse & 11.25 & 89.366 & 0.004 & 0.416 & 0.009 & 132.59 & 3.398 & 5 \\
\hline transverse & 0.25 & 89.567 & 0.007 & 0.719 & 0.015 & 91.015 & 2.213 & 5 \\
\hline transverse & 1.25 & 89.554 & 0.007 & 0.693 & 0.016 & 90.396 & 2.308 & 5 \\
\hline transverse & 2.25 & 89.483 & 0.004 & 0.449 & 0.009 & 152.886 & 3.541 & 5 \\
\hline transverse & 3.25 & 89.463 & 0.004 & 0.434 & 0.008 & 159.764 & 3.619 & 5 \\
\hline transverse & 4.25 & 89.585 & 0.004 & 0.504 & 0.01 & 143.121 & 3.212 & 5 \\
\hline transverse & 5.25 & 89.7 & 0.004 & 0.449 & 0.008 & 173.475 & 3.702 & 5 \\
\hline transverse & 6.25 & 89.747 & 0.004 & 0.442 & 0.007 & 182.478 & 3.808 & 5 \\
\hline transverse & 7.25 & 89.754 & 0.003 & 0.441 & 0.007 & 189.899 & 3.896 & 5 \\
\hline transverse & 8.25 & 89.704 & 0.003 & 0.392 & 0.006 & 213.31 & 4.333 & 5 \\
\hline transverse & 9.25 & 89.585 & 0.003 & 0.394 & 0.006 & 224.582 & 4.454 & 5 \\
\hline transverse & 10.25 & 89.467 & 0.003 & 0.395 & 0.006 & 228.225 & 4.433 & 5 \\
\hline transverse & 11.25 & 89.382 & 0.004 & 0.404 & 0.008 & 156.233 & 3.713 & 5 \\
\hline transverse & 0.25 & 89.566 & 0.003 & 0.358 & 0.007 & 180.145 & 4.143 & 5 \\
\hline transverse & 1.25 & 89.552 & 0.003 & 0.369 & 0.007 & 178.622 & 4.088 & 5 \\
\hline transverse & 2.25 & 89.549 & 0.003 & 0.402 & 0.007 & 178.058 & 3.915 & 5 \\
\hline transverse & 3.25 & 89.573 & 0.003 & 0.384 & 0.007 & 182.861 & 4.083 & 5 \\
\hline transverse & 4.25 & 89.611 & 0.003 & 0.405 & 0.007 & 181.993 & 3.965 & 5 \\
\hline transverse & 5.25 & 89.642 & 0.003 & 0.392 & 0.007 & 195.909 & 4.192 & 5 \\
\hline transverse & 6.25 & 89.663 & 0.003 & 0.387 & 0.006 & 209.689 & 4.297 & 5 \\
\hline transverse & 7.25 & 89.662 & 0.003 & 0.393 & 0.007 & 202.183 & 4.225 & 5 \\
\hline transverse & 8.25 & 89.635 & 0.003 & 0.379 & 0.006 & 228.514 & 4.5 & 5 \\
\hline transverse & 9.25 & 89.573 & 0.003 & 0.37 & 0.006 & 236.871 & 4.654 & 5 \\
\hline transverse & 10.25 & 89.498 & 0.003 & 0.382 & 0.006 & 226.833 & 4.487 & 5 \\
\hline transverse & 11.25 & 89.425 & 0.003 & 0.39 & 0.007 & 165.051 & 3.857 & 5 \\
\hline transverse & 0.25 & 89.534 & 0.003 & 0.36 & 0.006 & 189.801 & 4.218 & 5 \\
\hline transverse & 1.25 & 89.53 & 0.003 & 0.354 & 0.006 & 188.948 & 4.298 & 5 \\
\hline transverse & 2.25 & 89.527 & 0.003 & 0.357 & 0.006 & 194.966 & 4.305 & 5 \\
\hline
\end{tabular}




\begin{tabular}{|c|c|c|c|c|c|c|c|c|}
\hline transverse & 3.25 & 89.534 & 0.003 & 0.356 & 0.006 & 202.131 & 4.404 & 5 \\
\hline transverse & 4.25 & 89.533 & 0.003 & 0.351 & 0.006 & 214.875 & 4.562 & 5 \\
\hline transverse & 5.25 & 89.531 & 0.003 & 0.367 & 0.006 & 218.721 & 4.518 & 5 \\
\hline transverse & 6.25 & 89.532 & 0.003 & 0.358 & 0.006 & 217.364 & 4.565 & 5 \\
\hline transverse & 7.25 & 89.529 & 0.003 & 0.354 & 0.006 & 220.621 & 4.618 & 5 \\
\hline transverse & 8.25 & 89.529 & 0.003 & 0.34 & 0.006 & 235.047 & 4.846 & 5 \\
\hline transverse & 9.25 & 89.528 & 0.003 & 0.356 & 0.006 & 239.831 & 4.761 & 5 \\
\hline transverse & 10.25 & 89.531 & 0.003 & 0.35 & 0.005 & 244.3 & 4.859 & 5 \\
\hline transverse & 11.25 & 89.536 & 0.003 & 0.392 & 0.007 & 165.016 & 3.816 & 5 \\
\hline transverse & 864 & 89.561 & 0.003 & 0.335 & 0.006 & 220.944 & 4.766 & 5 \\
\hline transverse & 10. -60 & 89.561 & 0.003 & 0.333 & 0.006 & 220.093 & 4.769 & 5 \\
\hline transverse & 108 & 89.547 & 0.003 & 0.35 & 0.006 & 222.766 & 4.678 & 5 \\
\hline transverse & -52 & 89.531 & 0.003 & 0.346 & 0.006 & 207.162 & 4.535 & 5 \\
\hline transverse & 16ro 48 & 89.516 & 0.003 & 0.365 & 0.006 & 205.132 & 4.4 & 5 \\
\hline transverse & $3(10-24=4$ & 89.507 & 0.003 & 0.361 & 0.006 & 206.608 & 4.429 & 5 \\
\hline transverse & 18-40 & 89.512 & 0.003 & 0.36 & 0.006 & 207.512 & 4.424 & 5 \\
\hline transverse & $10-36$ & 89.513 & 0.003 & 0.347 & 0.006 & 209.008 & 4.577 & 5 \\
\hline transverse & $1.7-32$ & 89.517 & 0.003 & 0.357 & 0.006 & 216.211 & 4.512 & 5 \\
\hline transverse & $\begin{array}{r}28 \\
\end{array}$ & 89.52 & 0.003 & 0.376 & 0.006 & 197.475 & 4.259 & 5 \\
\hline transverse & $384=24$ & 89.555 & 0.003 & 0.37 & 0.006 & 199.784 & 4.288 & 5 \\
\hline transverse & (2) 20 & 89.582 & 0.003 & 0.354 & 0.006 & 208.815 & 4.472 & 5 \\
\hline transverse & 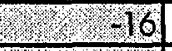 & 89.606 & 0.003 & 0.371 & 0.006 & 203.324 & 4.345 & 5 \\
\hline transverse & 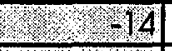 & 89.621 & 0.003 & 0.379 & 0.007 & 190.277 & 4.202 & 5 \\
\hline transverse & 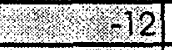 & 89.644 & 0.003 & 0.41 & 0.007 & 183.879 & 3.963 & 5 \\
\hline transverse & 410 & 89.662 & 0.004 & 0.417 & 0.008 & 163.694 & 3.738 & 5 \\
\hline transverse & 8 & 89.709 & 0.004 & 0.473 & 0.009 & 141.661 & 3.309 & 5 \\
\hline transverse & 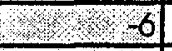 & 89.737 & 0.004 & 0.499 & 0.008 & 165.581 & 3.417 & 5 \\
\hline transverse & 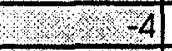 & 89.739 & 0.004 & 0.466 & 0.008 & 173.813 & 3.61 & 5 \\
\hline transverse & 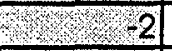 & 89.734 & 0.004 & 0.511 & 0.009 & 153.376 & 3.285 & 5 \\
\hline trancyerse & 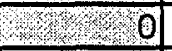 & 89.716 & 0.004 & 0.487 & 0.009 & 155.213 & 3.394 & 5 \\
\hline transyerse & 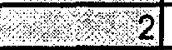 & 89.674 & 0.004 & 0.425 & 0.008 & 176.589 & 3.854 & 5 \\
\hline transverse & 48 & 89.654 & 0.003 & 0.394 & 0.007 & 191.666 & 4.15 & 5 \\
\hline transverse & 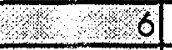 & 89.624 & 0.003 & 0.375 & 0.006 & 195.235 & 4.231 & 5 \\
\hline transverse & 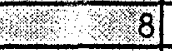 & 89.603 & 0.003 & 0.356 & 0.006 & 215.476 & 4.561 & 5 \\
\hline transverse & 12010 & 89.578 & 0.003 & 0.358 & 0.006 & 218.169 & 4.557 & 5 \\
\hline transverse & $\begin{array}{r}12 \\
\end{array}$ & 89.548 & 0.003 & 0.355 & 0.006 & 214.019 & 4.521 & 5 \\
\hline transverse & 0.14 & 89.529 & 0.003 & 0.343 & 0.006 & 218.345 & 4.704 & 5 \\
\hline transverse & $10 \quad 16$ & 89.531 & 0.003 & 0.355 & 0.006 & 219.417 & 4.605 & 5 \\
\hline transyarse & $3 \quad 20$ & 89.531 & 0.003 & 0.365 & 0.006 & 199.798 & 4.354 & 5 \\
\hline trans erse & $\begin{array}{r}24 \\
\end{array}$ & 89.527 & 0.003 & 0.35 & 0.006 & 207.924 & 4.544 & 5 \\
\hline trans erse & $3 \quad 28$ & 89.524 & 0.003 & 0.357 & 0.006 & 205.39 & 4.47 & 5 \\
\hline transverse & 32 & 89.533 & 0.003 & 0.374 & 0.007 & 189.236 & 4.205 & 5 \\
\hline transverse & 36 & 89.529 & 0.003 & 0.366 & 0.006 & 204.066 & 4.381 & 5 \\
\hline transverse & 40 & 89.529 & 0.003 & 0.361 & 0.006 & 201.745 & 4.427 & 5 \\
\hline transverse & $\begin{array}{r}1 \\
1\end{array}$ & 89.531 & 0.003 & 0.346 & 0.006 & 222.23 & 4.668 & 5 \\
\hline transverse & 48 & 89.542 & 0.003 & 0.361 & 0.006 & 199.003 & 4.386 & 5 \\
\hline transverse & 21 & 89.558 & 0.003 & 0.352 & 0.006 & 217.718 & 4.624 & 5 \\
\hline transverse & 56 & 89.573 & 0.003 & 0.357 & 0.006 & 212.054 & 4.501 & 5 \\
\hline transverse & 060 & 89.575 & 0.003 & 0.353 & 0.006 & 217.612 & 4.584 & 5 \\
\hline transverse & $\quad 64$ & 89.599 & 0.002 & 0.374 & 0.005 & 283.453 & 5.018 & 5 \\
\hline longitudina & 0.25 & 89.667 & 0.004 & 0.376 & 0.008 & 217.24 & 5.047 & \\
\hline longitudina & 1.25 & 89.632 & 0.003 & 0.38 & 0.006 & 318.43 & $\begin{array}{r}5.838 \\
\end{array}$ & \\
\hline
\end{tabular}




\begin{tabular}{|c|c|c|c|c|c|c|c|c|}
\hline longitudind & 2.25 & 89.630 & 0.003 & 0.391 & 0.006 & 307529 & 5.663 & 20 \\
\hline longituding & 3.25 & 89.624 & 0003 & 0.368 & 0.006 & 315.106 & 5.897 & 20 \\
\hline longitudina & 4.25 & 89.622 & 0.003 & 0.377 & 0.006 & 289.117 & 5.662 & \\
\hline longitudina & 5.25 & 89.619 & 0.003 & 0.384 & 0.007 & 280.469 & 5552 & 20 \\
\hline longitudina & 6.25 & 89.622 & 0.003 & 0.382 & 0.006 & 294.446 & 5.661 & 20 \\
\hline Iongitudina & 7.25 & 89.617 & 0.003 & 0.384 & 0.006 & 296.404 & 5.678 & 20 \\
\hline longitudina & 8.25 & 89.622 & 0.003 & 0.388 & 0.007 & 285123 & 5.57 & 20 \\
\hline longitudina & 9.25 & 89.623 & 0.003 & 0.382 & 0.006 & 318.747 & 5.818 & \\
\hline Iongitudina & 10.25 & 89.625 & 0.003 & 0.391 & 0.006 & 323.191 & 5.794 & 20 \\
\hline longit & 11.25 & & 0.003 & 0.376 & 0.006 & 323.539 & 5888 & 20 \\
\hline long & 0.25 & & 0.003 & 0.391 & 0.008 & 220.958 & 4.886 & 18 \\
\hline long & 7.25 & & 0.003 & 0.397 & 0.006 & 279.747 & 5.299 & 18 \\
\hline long & 2.25 & & & 0.385 & 0.007 & 273.444 & 5.357 & 18 \\
\hline long & 3.25 & & & 0.382 & 0.007 & 265.781 & 5.338 & 18 \\
\hline long & 4.25 & & 0.1 & 0.368 & 0.007 & 258.219 & 5.362 & 18 \\
\hline long & 5.25 & & & 0.375 & 0.007 & 248.684 & 5.278 & 18 \\
\hline long & 6.25 & 16 & 0.003 & 0.366 & 0.007 & 259.282 & 5.437 & 18 \\
\hline long & 7.25 & 402 & 0.0 & 0.384 & 0.007 & 261.706 & 5.309 & 18 \\
\hline long & 8.25 & & & 0.379 & 0.007 & 265.953 & 5.338 & 18 \\
\hline lons & 9.25 & & & 0.408 & 0.007 & 258.27 & 5.121 & 18 \\
\hline Ions & 10.25 & & & 0.425 & 0.007 & 242.218 & 4.854 & 18 \\
\hline $\overrightarrow{\text { Ions }}$ & .25 & & & 0.433 & 0.008 & 226.022 & 4.705 & 18 \\
\hline Iong & 0.25 & & & 0.398 & 0.008 & 208.486 & 4.761 & 18 \\
\hline Ions & 1.25 & & 0.003 & 0.41 & 0.007 & 253.17 & 5.058 & 18 \\
\hline ina & 2.25 & & & 0.407 & 0.007 & 246.329 & $\begin{array}{r}4.993 \\
\end{array}$ & 18 \\
\hline dina & 3.25 & 373 & 03 & 0.422 & 0.008 & 236.29 & 4.862 & 18 \\
\hline ine & 4.25 & & & 0.424 & 0.008 & 228.486 & 4.8 & 18 \\
\hline ina & 5.25 & & & 0.44 & 0.008 & 232162 & 4.736 & 18 \\
\hline ina & 6.25 & & 03 & 0.424 & 0.008 & 235.443 & 4785 & 18 \\
\hline ina & 7.25 & & & 0.425 & 0.008 & 231.987 & 4.792 & 18 \\
\hline ding & 8.25 & 286 & 0. & 0.45 & 0.009 & 214.994 & 4.529 & 18 \\
\hline long & 9.25 & 268 & 0.0 & 0.5 & 10.01 & 200.488 & 4208 & 18 \\
\hline Iong & 10.25 & & & 0.56 & 0.01 & 192.771 & 3.873 & 18 \\
\hline & .25 & & & 0.562 & 0.01 & 185.281 & 3.814 & 18 \\
\hline & 25 & 399 & 04 & 0.402 & 0.009 & 172.554 & 4.408 & 18 \\
\hline & 1.25 & & & 0.403 & 0.007 & 244.017 & 5.013 & 18 \\
\hline & 2.25 & & & 0.436 & 0.008 & 218.399 & 4.636 & 18 \\
\hline & 3.25 & 345 & & 0.467 & 0.009 & 199.425 & 4.319 & 18 \\
\hline & 4.25 & 353 & & 0.486 & 0.005 & 198.544 & 4.218 & 18 \\
\hline lina & 5.25 & & 04 & 0.438 & 0.008 & 217.192 & 4.634 & 18 \\
\hline idina & 6.25 & 296 & 104 & 0.43 & 0.008 & 221.336 & 4.708 & 18 \\
\hline udina & 7.25 & 250 & 003 & 0.416 & 0.008 & 235.464 & 4.878 & 18 \\
\hline udina & 8.25 & 221 & & 0.422 & 0.008 & 218.632 & 4.683 & 18 \\
\hline udina & 9.25 & 230 & 004 & 0.498 & 0.01 & 183.965 & 4.058 & 18 \\
\hline idina & 10.25 & 277 & 005 & 0.59 & 0.012 & 171.729 & 3.628 & 18 \\
\hline dina & 11.25 & 325 & 05 & 0.614 & 0.012 & 174.141 & 3.574 & 18 \\
\hline dina & 0.25 & 89.685 & 0.004 & 0.359 & 0.009 & 163.909 & 4.587 & 18 \\
\hline dina & 1.25 & 89.631 & 0,003 & 0.354 & 0.006 & 298.256 & 5813 & 18 \\
\hline dina & 2.25 & 89.611 & 0.003 & 0.333 & 0.005 & 317.127 & 6.13 & 18 \\
\hline & 3.25 & 89.606 & 0.003 & 0.352 & 0.006 & 288.57 & 5775 & 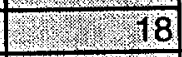 \\
\hline & 4.25 & 89.606 & 0.003 & 0.349 & 0.006 & 290.655 & 5.834 & \\
\hline & 5.25 & 89.607 & 0.003 & 0.367 & 0.006 & 281.353 & 5.618 & \\
\hline
\end{tabular}




\begin{tabular}{|c|c|c|c|c|c|c|c|c|}
\hline longitudina & 6.25 & 89.606 & 0.003 & 0,36 & 0.006 & 282.175 & 5.673 & 18 \\
\hline Iongitudina & 7.25 & 89.608 & 80.003 & 0.353 & 0.006 & $300: 834$ & 5.875 & 418 \\
\hline longitudina & 8.25 & 89.602 & 80.003 & 0.354 & 0.006 & $293: 233$ & 5.795 & 2018 \\
\hline longitudina & 9.25 & 89610 & 0.003 & 0.359 & 0,006 & 296.936 & 5.843 & 18 \\
\hline longitudina & 10.25 & $89: 600$ & 0.003 & 0.352 & 0.006 & 325.03 & 6.053 & 12018 \\
\hline longitudina & 11,25 & 89.607 & 0.003 & 0.362 & 0.006 & 313.617 & 5.845 & 18 \\
\hline longitudina & 0.25 & 89.475 & 0.005 & 0.382 & 0.013 & 115.455 & 3.953 & 18 \\
\hline Iongituding & 1.25 & 89.445 & 0.003 & 0.399 & 0.007 & 248.266 & 5.101 & 18 \\
\hline longitudina & 2.25 & 89.439 & 0.003 & 0.387 & 0.006 & 273.831 & 5.324 & 18 \\
\hline longitudina & 3.25 & 89.438 & 0.003 & 0.38 & 0.007 & 260.849 & 5.309 & 18 \\
\hline Iongitudina & 4.25 & 89.446 & 0.003 & 0.38 & 0.007 & 268.191 & 5.366 & 18 \\
\hline longitudina & 5.25 & 89.440 & 0.003 & 0.38 & 0.007 & 264.379 & 5.369 & 18 \\
\hline longitudina & 6.25 & 89.427 & 0.003 & 0.381 & 0.007 & 262.904 & 5.314 & 18 \\
\hline longitudina & 7.25 & 89.416 & 0.003 & 0.376 & 0.007 & 263.177 & 5.379 & 18 \\
\hline Iongitudina & 8.25 & 89.404 & 0.003 & 0.385 & 0.007 & 268.222 & 5.331 & 18 \\
\hline longitudina & 9.25 & 89.387 & 0.003 & 0.404 & 0.007 & 264.807 & 5.17 & 18 \\
\hline longitudina & 10.25 & 89.383 & 0.003 & 0.392 & 0.006 & 283.547 & 5.372 & 18 \\
\hline longitudina & 11.25 & 89.377 & 0.003 & 0.391 & 0.007 & 281.901 & 5.385 & 18 \\
\hline longitudina & 0.25 & 89430 & 0.005 & 0.389 & 0.012 & 116.957 & 3.878 & 18 \\
\hline longitudina & 1.25 & 89.403 & 0.003 & 0.416 & 0.007 & 235.29 & 4816 & 18 \\
\hline longitudina & 2.25 & 89.406 & 0.003 & 0.406 & 0.007 & 247.408 & 5.025 & 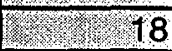 \\
\hline longitudina & 3.25 & 89.421 & 0.003 & 0.396 & 0.007 & 254.213 & 5.145 & W 18 \\
\hline longitudina & 4.25 & 89.421 & 0.003 & 0.404 & 0.007 & 242,452 & 4.989 & W/18 \\
\hline longitudina & 5.25 & 89.405 & 0.003 & 0.408 & 0.008 & 230.507 & 4.909 & \% \\
\hline longitudina & 6.25 & 89.378 & 0.0004 & 0.429 & 0.008 & 218.085 & 4.687 & 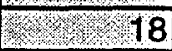 \\
\hline Iongitudina & 7.25 & 89.351 & 0,004 & 0.441 & 0.008 & 217962 & 4598 & 18 \\
\hline longitudina & 8.25 & 89.329 & 0.004 & 0.43 & 0.008 & 217855 & 4.674 & 18 \\
\hline longitudina & 9.25 & 89.337 & 0.004 & 0.446 & 0.008 & 220.378 & 4.606 & 18 \\
\hline longituding & 10.25 & 89.364 & 0.004 & 0.467 & 0.009 & 208.031 & 4.433 & $18 \times 18$ \\
\hline longitudina & 11.25 & 89.388 & 0.005 & 0.596 & 0.012 & 169.777 & 3.652 & 18 \\
\hline Iongitudina & 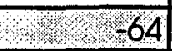 & 89.536 & 0.003 & 0.392 & 0.007 & 244.773 & 5.111 & 18 \\
\hline longitudina & 80 & 89.513 & 0.003 & 0.385 & 0.007 & 264.253 & 5.324 & 18 \\
\hline Iongitudina & 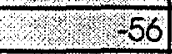 & 89.501 & 0.003 & 0.376 & 0.007 & 265.491 & 5.398 & 18 \\
\hline longitudina & $74 \quad 52$ & 89.523 & 0.003 & 0.389 & 0.007 & 256.47 & 5.229 & 18 \\
\hline longitudina & $76-48$ & 89.564 & 0.003 & 0.386 & 0.007 & 258.861 & 5.302 & 18 \\
\hline Iongitudina & $\therefore \quad 44$ & 89.617 & 0.003 & 0.375 & 0.006 & 269.641 & 5.426 & 18 \\
\hline Iongitudina & 34240 & 89.657 & 0.003 & 0.373 & 0.007 & 269.347 & 5.483 & 18 \\
\hline longitudina & -36 & 89.663 & 0.003 & 0.358 & 0.006 & 282.773 & 5.705 & 18 \\
\hline longitudina & rex -32 & 89.672 & 0.003 & 0.367 & 0.007 & 270.495 & 5.564 & 18 \\
\hline Iongitudina & (3) -28 & 89.669 & 0.003 & 0.354 & 0.006 & 263.664 & 5.592 & 18 \\
\hline longitudina & 24 & 89.647 & 0.003 & 0.376 & 0.007 & 261.457 & 5.396 & 18 \\
\hline longitudine & Wry 20 & 89.614 & 0.003 & 0.36 & 0.006 & 267.695 & 5.561 & 18 \\
\hline longitudina & 15y -16 & 89.553 & 0.003 & 0.369 & 0.007 & 263.027 & 5.433 & 18 \\
\hline longitudina & $4 \times 14$ & 89.472 & 0.003 & 0.368 & 0.007 & 256.225 & 5.37 & 18 \\
\hline Iongitudina & 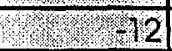 & 89.450 & 0.003 & 0.363 & 0.007 & 265.048 & 5.501 & 18 \\
\hline Iongituding & 1. & 89.413 & 0.003 & 0.371 & 0.007 & 258.264 & 5.355 & 18 \\
\hline longitudina & 167\% 8 & 89.345 & 0.003 & 0.408 & 0.007 & 241.768 & 4.975 & 18 \\
\hline longitudina & $7 x+6$ & 89.294 & 0.003 & 0.419 & 0.008 & 244.991 & 4.967 & 18 \\
\hline longitudina & -4 & 89.289 & 0.003 & 0.428 & 0.008 & 237.423 & 4.839 & $\overline{18}$ \\
\hline Iongitudina & -2 & 89.292 & 0.004 & 0.428 & 0.008 & 226.636 & 4.741 & 18 \\
\hline Iongitudina & 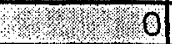 & 89.300 & 0.004 & 0.447 & 0.008 & 215.506 & 4.574 & 18 \\
\hline longitudina & 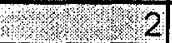 & 89.357 & 0.004 & 0.462 & 0.009 & 211.035 & 4.457 & 18 \\
\hline
\end{tabular}




\begin{tabular}{|l|r|r|r|r|r|r|r|r|}
\hline longitudina & & 89.387 & 0.004 & 0.416 & 0.008 & 227.414 & 4.833 & 18 \\
\hline longitudina & 6 & 89.423 & 0.003 & 0.395 & 0.007 & 252.281 & 5.139 & 18 \\
\hline longitudina & 8 & 89.445 & 0.003 & 0.38 & 0.007 & 265.938 & 5.395 & 18 \\
\hline longitudina & 10 & 89.479 & 0.003 & 0.377 & 0.007 & 267.889 & 5.423 & 18 \\
\hline longitudina & 12 & 89.525 & 0.003 & 0.399 & 0.007 & 257.42 & 5.195 & 18 \\
\hline longitudina & 14 & 89.562 & 0.003 & 0.386 & 0.007 & 256.448 & 5.285 & 18 \\
\hline longitudina & 16 & 89.589 & 0.003 & 0.378 & 0.007 & 258.986 & 5.377 & 18 \\
\hline longitudina & 20 & 89.626 & 0.003 & 0.375 & 0.007 & 273.636 & 5.502 & 18 \\
\hline longitudina & 24 & 89.619 & 0.003 & 0.37 & 0.006 & 280.664 & 5.603 & 18 \\
\hline longitudina & 28 & 89.612 & 0.003 & 0.353 & 0.006 & 287.317 & 5.756 & 18 \\
\hline longitudina & 32 & 89.613 & 0.003 & 0.348 & 0.006 & 290.49 & 5.844 & 18 \\
\hline longitudina & 36 & 89.612 & 0.003 & 0.359 & 0.006 & 284.915 & 5.692 & 18 \\
\hline longitudina & 40 & 89.606 & 0.003 & 0.351 & 0.006 & 279.382 & 5.743 & 18 \\
\hline longitudina & 44 & 89.608 & 0.003 & 0.357 & 0.006 & 283.804 & 5.738 & 18 \\
\hline longitudina & 48 & 89.603 & 0.003 & 0.347 & 0.006 & 294.07 & 5.873 & 18 \\
\hline longitudina & 52 & 89.598 & 0.003 & 0.366 & 0.006 & 285.543 & 5.639 & 18 \\
\hline longitudina & 56 & 89.587 & 0.003 & 0.358 & 0.006 & 282.413 & 5.694 & 18 \\
\hline longitudina & 60 & 89.530 & 0.003 & 0.34 & 0.006 & 297.111 & 5.938 & 18 \\
\hline longitudina & 64 & 89.488 & 0.003 & 0.358 & 0.006 & 276.805 & 5.62 & 18 \\
\hline
\end{tabular}




\section{Detector \#3}

\section{Ferritic Steel D-zero Comb-like Sample}

\begin{tabular}{|c|c|c|c|c|c|c|c|c|}
\hline direction & $\begin{array}{c}\text { Position } \\
\text { (mm) }\end{array}$ & $\begin{array}{c}2 \Theta / d / a \\
{[\circ / A]}\end{array}$ & uncertainty & $\begin{array}{c}\text { FWHM } \\
{\left[{ }^{\circ}\right]}\end{array}$ & \begin{tabular}{|c|} 
uncertainty \\
{$\left[{ }^{\circ}\right]$}
\end{tabular} & \begin{tabular}{|c|} 
Peak \\
Height
\end{tabular} & uncertainty & $\begin{array}{l}\text { sampling } \\
\text { time (min.) }\end{array}$ \\
\hline normal & 0.25 & 89.559 & 0.003 & 0.35 & 0.008 & 283.07 & 6.317 & 50 \\
\hline normal & 1.25 & 89.56 & 0.003 & 0.347 & 0.007 & 254.73 & 5.682 & 30 \\
\hline normal & 2.25 & 89.573 & 0.004 & 0.342 & 0.009 & 156.2 & 4.348 & 15 \\
\hline normal & 3.25 & 89.569 & 0.004 & 0.347 & 0.008 & 155.07 & 4.19 & 12 \\
\hline normal & 4.25 & 89.562 & 0.003 & 0.341 & 0.007 & 166.53 & 4.228 & 10 \\
\hline normal & 5.25 & 89.566 & 0.004 & 0.359 & 0.008 & 156.86 & 3.982 & 8 \\
\hline normal & 6.25 & 89.561 & 0.003 & 0.346 & 0.006 & 213.56 & 4.627 & 9 \\
\hline normal & 7.25 & 89.562 & 0.003 & 0.362 & 0.005 & 274.08 & 5.101 & 10 \\
\hline normal & 8.25 & 89.562 & 0.002 & 0.349 & 0.004 & 384.66 & 6.111 & 12 \\
\hline normal & 9.25 & 89.553 & 0.002 & 0.358 & 0.004 & 564.18 & 7.258 & 15 \\
\hline normal & 10.25 & 89.553 & 0.001 & 0.355 & 0.002 & 1495.3 & 11.733 & 30 \\
\hline normal & 11.25 & 89.559 & 0.001 & 0.361 & 0.001 & 3078.7 & 16.575 & 50 \\
\hline normal & 0.25 & 89.564 & 0.006 & 0.657 & 0.013 & 213.83 & 4.294 & 50 \\
\hline normal & 1.25 & 89.563 & 0.004 & 0.475 & 0.01 & 209.05 & 4.567 & 30 \\
\hline normal & 2.25 & 89.559 & 0.005 & 0.439 & 0.01 & 143.85 & 3.72 & 15 \\
\hline normal & 3.25 & 89.558 & 0.004 & 0.397 & 0.009 & 159.27 & 3.968 & 12 \\
\hline normal & 4.25 & 89.565 & 0.004 & 0.4 & 0.008 & \begin{tabular}{|l|}
160.09 \\
\end{tabular} & 3.875 & 10 \\
\hline normal & 5.25 & 89.573 & 0.004 & 0.411 & 0.008 & 160.47 & 3.736 & 8 \\
\hline normal & 6.25 & 89.563 & 0.003 & 0.4 & 0.007 & 205.73 & 4.259 & 9 \\
\hline normal & 7.25 & 89.573 & 0.003 & 0.407 & 0.006 & 254.65 & 4.673 & 10 \\
\hline normal & 8.25 & 89.581 & 0.002 & 0.392 & 0.005 & 361.16 & 5.643 & 12 \\
\hline normal & 9.25 & 89.571 & 0.002 & 0.395 & 0.004 & 569.55 & 6.987 & 15 \\
\hline normal & 10.25 & 89.572 & 0.001 & 0.389 & 0.002 & 1491.3 & 11.239 & 30 \\
\hline normal & 11.25 & 89.573 & 0.001 & 0.401 & 0.002 & 2728.7 & 14.945 & 50 \\
\hline normal & 0.25 & 89.554 & 0.004 & 0.517 & 0.009 & 283.99 & 5.221 & 50 \\
\hline normal & 1.25 & 89.549 & 0.004 & 0.51 & 0.01 & 218.99 & 4.459 & 30 \\
\hline normal & 2.25 & 89.557 & 0.005 & 0.522 & 0.011 & 143.36 & 3.388 & 15 \\
\hline normal & 3.25 & 89.571 & 0.005 & 0.483 & 0.01 & \begin{tabular}{|l|}
144.77 \\
\end{tabular} & 3.458 & 12 \\
\hline normal & 4.25 & 89.568 & 0.004 & 0.403 & 0.008 & 166.44 & 3.934 & 10 \\
\hline normal & 5.25 & 89.569 & 0.004 & 0.45 & 0.009 & 152.43 & 3.52 & 8 \\
\hline normal & 6.25 & 89.566 & 0.004 & 0.49 & 0.008 & 181.77 & 3.663 & 9 \\
\hline normal & 7.25 & 89.572 & 0.004 & 0.495 & 0.008 & 209.57 & 3.926 & 10 \\
\hline normal & 8.25 & 89.577 & 0.003 & 0.456 & 0.006 & 322.19 & 5.009 & 12 \\
\hline normal & 9.25 & 89.566 & 0.002 & 0.429 & 0.004 & 525.63 & 6.469 & 15 \\
\hline normal & 10.25 & 89.575 & 0.001 & 0.42 & 0.002 & 1364.7 & 10.427 & 30 \\
\hline normal & 11.25 & 89.569 & 0.001 & 0.429 & 0.002 & 2405.7 & 13.713 & 50 \\
\hline normal & 0.25 & 89.553 & 0.004 & 0.669 & 0.01 & 321.44 & 4.775 & 50 \\
\hline normal & 1.25 & 89.553 & 0.004 & 0.593 & 0.01 & 241.5 & 4.274 & 30 \\
\hline normal & 2.25 & 89.562 & 0.004 & 0.497 & \begin{tabular}{l|l}
0.009 \\
\end{tabular} & 176.75 & 3.786 & 15 \\
\hline normal & 3.25 & 89.554 & 0.004 & 0.452 & 0.009 & \begin{tabular}{|l|}
174.9 \\
\end{tabular} & 3.883 & 12 \\
\hline normal & 4.25 & 89.568 & 0.004 & 0.452 & 0.009 & 160.56 & 3.637 & 10 \\
\hline normal & 5.25 & 89.56 & 0.004 & 0.464 & 0.009 & 157.73 & 3.564 & 9 \\
\hline normal & 6.25 & 89.571 & 0.004 & 0.46 & 0.009 & 160.55 & 3.606 & 8 \\
\hline normal & 7.25 & 89.577 & 0.004 & 0.461 & 0.009 & 138.65 & 3.291 & 6 \\
\hline normal & 8.25 & 89.568 & 0.005 & 0.473 & 0.01 & 109.14 & 2.847 & 4 \\
\hline normal & 9.25 & 89.565 & 0.004 & 0.468 & 0.009 & 120.03 & 2.943 & 3.5 \\
\hline
\end{tabular}




\begin{tabular}{|c|c|c|c|c|c|c|c|c|}
\hline normal & 10.25 & 89.572 & 0.004 & 0.419 & 0.008 & 140.392 & 3.324 & 3 \\
\hline normal & 11.25 & 89.563 & 0.004 & 0.436 & 0.008 & 127.496 & 3.085 & 3 \\
\hline normal & 0.25 & 89.547 & 0.005 & 0.733 & 0.012 & 257.175 & 4.258 & 50 \\
\hline normal & 1.25 & 89.555 & 0.005 & 0.631 & 0.012 & 200.279 & 3.908 & 30 \\
\hline normal & 2.25 & 89.564 & 0.004 & 0.469 & 0.01 & 161.497 & 3.791 & 15 \\
\hline normal & 3.25 & 89.563 & 0.004 & 0.419 & 0.008 & 175.349 & 4.01 & 12 \\
\hline normal & 4.25 & 89.569 & 0.004 & 0.434 & 0.009 & 156.068 & 3.708 & 10 \\
\hline normal & 5.25 & 89.57 & 0.004 & 0.431 & 0.008 & 168.576 & 3.802 & 9 \\
\hline normal & 6.25 & 89.569 & 0.004 & 0.435 & 0.008 & 185.628 & 3.908 & 8 \\
\hline normal & 7.25 & 89.57 & 0.004 & 0.436 & 0.008 & 152.817 & 3.505 & 6 \\
\hline normal & 8.25 & 89.569 & 0.004 & 0.416 & 0.008 & 123.485 & 3.188 & 4 \\
\hline normal & 9.25 & 89.569 & 0.004 & 0.412 & 0.007 & 148.786 & 3.458 & 3.5 \\
\hline normal & 70.25 & 89.572 & 0.003 & 0.406 & 0.007 & 154.075 & 3.518 & 3 \\
\hline normal & 11.25 & 89.57 & 0.004 & 0.421 & 0.008 & 129.205 & 3.217 & 3 \\
\hline normal & 0.25 & 89.563 & 0.002 & 0.375 & 0.005 & 491.448 & 7.4 & 50 \\
\hline normal & 1.25 & 89.553 & 0.003 & 0.387 & 0.006 & 350.969 & 6.063 & 30 \\
\hline normal & 2.25 & 89.56 & 0.003 & 0.392 & 0.007 & 209.451 & 4.533 & 15 \\
\hline normal & 3.25 & 89.562 & 0.003 & 0.388 & 0.007 & 199.783 & 4.382 & 12 \\
\hline normal & 4.25 & 89.571 & 0.003 & 0.381 & 0.007 & 200.707 & 4.371 & 10 \\
\hline normal & 5.25 & 89.569 & 0.003 & 0.365 & 0.006 & 216.134 & 4.564 & 9 \\
\hline normal & 6.25 & 89.566 & 0.003 & 0.372 & 0.006 & 206.286 & 4.4 & 8 \\
\hline normal & 7.25 & 89.565 & 0.003 & 0.376 & 0.007 & 181.918 & 4.09 & 6 \\
\hline normal & 8.25 & 89.572 & 0.004 & 0.387 & 0.008 & 129.505 & 3.369 & 4 \\
\hline normal & 9.25 & 89.56 & 0.004 & 0.385 & 0.007 & 143.986 & 3.543 & 3.5 \\
\hline normal & 10.25 & 89.562 & 0.003 & 0.383 & 0.006 & 161.05 & 3.691 & 3 \\
\hline normal & 11.25 & 89.561 & 0.003 & 0.41 & 0.007 & 155.78 & 3.504 & 3 \\
\hline normal & 0.25 & 89.55 & 0.004 & 0.365 & 0.008 & 258.082 & 6.014 & 50 \\
\hline normal & 1.25 & 89.549 & 0.004 & 0.37 & 0.008 & 217.095 & 5.177 & 30 \\
\hline normal & 2.25 & 89.556 & 0.004 & 0.38 & 0.01 & 141.331 & 3.952 & 15 \\
\hline normal & 3.25 & 89.562 & 0.004 & 0.363 & 0.008 & 156.509 & 4.098 & 12 \\
\hline normal & 4.25 & 89.561 & 0.003 & 0.363 & 0.007 & 167.9 & 4.112 & 10 \\
\hline normal & 5.25 & 89.564 & 0.003 & 0.357 & 0.007 & 185.68 & 4.27 & 9 \\
\hline normal & 6.25 & 89.564 & 0.003 & 0.363 & 0.006 & 207.714 & 4.461 & 8 \\
\hline normal & 7.25 & 89.563 & 0.003 & 0.368 & 0.006 & 186.875 & 4.111 & 6 \\
\hline normal & 8.25 & 89.56 & 0.003 & 0.341 & 0.007 & 144.595 & 3.745 & 4 \\
\hline normal & 9.25 & 89.558 & 0.003 & 0.344 & 0.007 & 148.208 & 3.746 & 3.5 \\
\hline normal & 10.25 & 89.553 & 0.003 & 0.343 & 0.006 & 159.367 & 3.879 & 3 \\
\hline normal & 11.25 & 89.563 & 0.003 & 0.358 & 0.006 & 193.997 & 4.164 & 3 \\
\hline
\end{tabular}


Detector \#4

Ferritic Steel Weld Plate

\begin{tabular}{|c|c|c|c|c|c|c|c|c|}
\hline direction & $\begin{array}{c}\text { Position } \\
\text { (mm) }\end{array}$ & $\begin{array}{c}2 \theta / d / a \\
{[\circ / A]}\end{array}$ & uncertainty & $\begin{array}{c}\text { FWHM } \\
{\left[{ }^{\circ}\right]}\end{array}$ & $\begin{array}{c}\text { uncertainty } \\
{\left[{ }^{\circ}\right]}\end{array}$ & $\begin{array}{c}\text { Peak } \\
\text { Height }\end{array}$ & uncertainty & $\begin{array}{c}\text { sampling } \\
\text { time }\end{array}$ \\
\hline normal & 0.25 & 89.577 & 0.007 & \begin{tabular}{|l|}
0.396 \\
\end{tabular} & \begin{tabular}{|r|}
0.016 \\
\end{tabular} & 86.317 & 3.485 & 18 \\
\hline normal & 1.25 & 89.596 & 0.005 & 0.413 & 0.012 & 123.56 & 3.683 & 15 \\
\hline normal & 2.25 & 89.598 & 0.005 & 0.402 & 0.01 & 124.72 & 3.568 & ter? 10 \\
\hline normal & 3.25 & 89.605 & 0.004 & 0.4 & 0.009 & 149.98 & 3.82 & 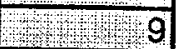 \\
\hline normal & 4.25 & 89.608 & 0.004 & 0.402 & 0.009 & 138.27 & 3.557 & 606 \\
\hline normal & 5.25 & 89.61 & 0.004 & 0.389 & 0.01 & 97.609 & 2.99 & एकी 3 \\
\hline normal & 6.25 & 89.616 & 0.003 & 0.401 & 0.007 & 182.5 & 3.916 & 1010? \\
\hline normal & 7.25 & 89.615 & 0.002 & 0.408 & 0.005 & 376.44 & 5.491 & पै। 6 \\
\hline normal & 8.25 & 89.616 & 0.002 & 0.404 & 0.003 & 767.02 & 7.838 & प) 9 \\
\hline normal & 9.25 & 89.624 & 0.001 & 0.396 & 0.002 & \begin{tabular}{|l|}
1148 \\
\end{tabular} & 9.572 & 10 \\
\hline normal & 10.25 & 89.626 & 0.001 & 0.404 & 0.002 & \begin{tabular}{|l|}
2281.6 \\
\end{tabular} & 13.314 & 15 \\
\hline normal & 11.25 & 89.633 & 0.001 & 0.401 & 0.001 & 3632.9 & 16.76 & 18 \\
\hline normal & 0.25 & 89.674 & 0.007 & 0.451 & 0.017 & 85.197 & 3.245 & 18 \\
\hline normal & 1.25 & 89.677 & 0.005 & 0.437 & 0.011 & 132.63 & 3.664 & 15 \\
\hline normal & 2.25 & 89.678 & 0.004 & 0.391 & 0.01 & 128.89 & 3.646 & 10 \\
\hline normal & 3.25 & 89.674 & 0.004 & 0.418 & 0.009 & 157.25 & 3.78 & 9 \\
\hline normal & 4.25 & 89.663 & 0.004 & 0.4 & 0.008 & 151.07 & 3.684 & 6 \\
\hline normal & 5.25 & 89.652 & 0.005 & 0.417 & 0.01 & 100.72 & 2.894 & 3 \\
\hline normal & 6.25 & 89.638 & 0.003 & 0.429 & 0.007 & 186.62 & 3.824 & 4 \\
\hline normal & 7.25 & 89.634 & 0.002 & 0.425 & 0.005 & 360.75 & 5.318 & 6 \\
\hline normal & 8.25 & 89.645 & 0.002 & 0.421 & 0.003 & 758.36 & 7.659 & 9 \\
\hline normal & 9.25 & 89.665 & 0.001 & 0.419 & 0.003 & 1123.5 & 9.262 & 10 \\
\hline normal & 10.25 & 89.693 & 0.001 & 0.415 & 0.002 & 2227.3 & 13.008 & 15 \\
\hline normal & 11.25 & 89.727 & 0.001 & 0.416 & 0.001 & 3516.9 & 16.225 & 8 \\
\hline normal & 0.25 & 89.732 & 0.01 & 0.587 & 0.025 & 68.661 & 2.716 & 8 \\
\hline normal & 1.25 & 89.746 & 0.007 & 0.607 & 0.017 & \begin{tabular}{|l|}
98.043 \\
\end{tabular} & 2.843 & 15 \\
\hline normal & 2.25 & 89.751 & 0.008 & 0.668 & 0.019 & 81.081 & 2.374 & 70 \\
\hline normal & 3.25 & 89.737 & 0.005 & 0.503 & 0.012 & 125.32 & 3.23 & 9 \\
\hline normal & 4.25 & 89.736 & 0.005 & 0.472 & 0.01 & 130.96 & 3.205 & 6 \\
\hline normal & 5.25 & 89.714 & 0.005 & 0.481 & 0.012 & \begin{tabular}{|l|}
86.941 \\
\end{tabular} & 2.546 & 3 \\
\hline normal & 6.25 & 89.668 & 0.004 & 0.495 & 0.009 & \begin{tabular}{|l|}
159.1 \\
\end{tabular} & 3.364 & 4 \\
\hline normal & 7.25 & 89.653 & 0.003 & \begin{tabular}{l|l}
0.472 \\
\end{tabular} & 0.006 & \begin{tabular}{|l|}
332.87 \\
\end{tabular} & 4.9 & 6 \\
\hline normal & 8.25 & 89.654 & 0.002 & 0.459 & 0.004 & 688.64 & 7.045 & 9 \\
\hline normal & 9.25 & 89.674 & 0.001 & 0.446 & 0.003 & 1066.3 & 8.773 & 10 \\
\hline normal & 10.25 & 89.723 & 0.001 & 0.441 & 0.002 & \begin{tabular}{|l|}
2090.3 \\
\end{tabular} & 12.258 & 15 \\
\hline normal & 11.25 & 89.763 & 0.001 & 0.441 & 0.001 & \begin{tabular}{|r|}
3252 \\
\end{tabular} & 15.22 & 18 \\
\hline normal & 0.25 & 89.651 & 0.008 & 0.65 & 0.02 & \begin{tabular}{|l|}
93.507 \\
\end{tabular} & 2.811 & 18 \\
\hline normal & 1.25 & 89.674 & 0.008 & 0.66 & 0.018 & \begin{tabular}{|l|}
99.521 \\
\end{tabular} & 2.742 & 15 \\
\hline norma! & 2.25 & 89.726 & 0.006 & 0.506 & 0.014 & 107.65 & 3.078 & 10 \\
\hline normel & 3.25 & 89.745 & 0.004 & 0.471 & 0.01 & \begin{tabular}{|l|}
156.32 \\
\end{tabular} & 3.604 & 9 \\
\hline normal & 4.25 & 89.734 & 0.004 & 0.476 & 0.009 & 143.09 & 3.337 & 6 \\
\hline normal & 5.25 & 89.692 & 0.005 & 0.5 & 0.011 & 93.071 & 2.565 & 3 \\
\hline normal & 6.25 & 89.665 & 0.004 & 0.494 & 0.009 & 162.24 & 3.388 & 4 \\
\hline normal & 7.25 & 89.635 & 0.003 & 0.529 & 0.006 & 311.31 & 4.467 & 6 \\
\hline normal & 8.25 & 89.643 & 0.002 & 0.547 & 0.004 & 579.63 & 5.987 & 9 \\
\hline normal & 9.25 & 89.69 & 0.002 & 0.506 & 0.003 & \begin{tabular}{|c|}
926.57 \\
\end{tabular} & 7.768 & 10 \\
\hline normal & 10.25 & 89.746 & 0.001 & 0.471 & \begin{tabular}{l|l}
0.002 \\
\end{tabular} & \begin{tabular}{|l|}
1939.7 \\
\end{tabular} & 11.474 & 15 \\
\hline
\end{tabular}




\begin{tabular}{|c|c|c|c|c|c|c|c|c|}
\hline normal & 11.25 & 89.783 & 0.001 & 0.466 & 0.002 & 3033.364 & 14.311 & 18 \\
\hline normal & 0.25 & 89.653 & 0.009 & 0.691 & 0.022 & 83.505 & 2.625 & 18 \\
\hline normal & 1.25 & 89.644 & 0.007 & 0.627 & 0.017 & 101.576 & 2.864 & 15 \\
\hline normal & 2.25 & 89.692 & 0.008 & 0.616 & 0.018 & 89.49 & 2.608 & 10 \\
\hline normal & 3.25 & 89.744 & 0.005 & 0.539 & 0.011 & 138.157 & 3.212 & 9 \\
\hline normal & 4.25 & 89.734 & 0.004 & 0.485 & 0.01 & 141.621 & 3.292 & 6 \\
\hline normal & 5.25 & 89.684 & 0.005 & 0.508 & 0.011 & 97,054 & 2.605 & 3 \\
\hline normal & 6.25 & 89.626 & 0.004 & 0.516 & 0.008 & 170.581 & 3.387 & 4. \\
\hline normal & 7.25 & 89.604 & 0.003 & 0.529 & 0.006 & 321.887 & 4.559 & 6 \\
\hline normal & 8.25 & 89.612 & 0.002 & 0.509 & 0.004 & 635.192 & 6.468 & 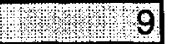 \\
\hline normal & 9.25 & 89.664 & 0.001 & 0.489 & 0.003 & 978.381 & 8.088 & 10 \\
\hline normal & 10.25 & 89.727 & 0.001 & 0.463 & 0.002 & 2043.581 & 11.873 & 15 \\
\hline normal & 11.25 & 89.77 & 0.001 & 0.459 & 0.002 & 3209.792 & 14.831 & 18 \\
\hline normal & 0.25 & 89.615 & 0.007 & 0.428 & 0.015 & 97.341 & 3.501 & 18 \\
\hline normal & 1.25 & 89.647 & 0.005 & 0.448 & 0.012 & 127.343 & 3.614 & 15 \\
\hline normal & 2.25 & 89.683 & 0.005 & 0.438 & 0.01 & 133.887 & 3.511 & 10 \\
\hline normal & 3.25 & 89.712 & 0.004 & 0.437 & 0.009 & 160.423 & 3.749 & 9 \\
\hline normal & 4.25 & 89.704 & 0.004 & 0.433 & 0.009 & 146.972 & 3.542 & 6 \\
\hline normal & 5.25 & 89.699 & 0.005 & 0.446 & 0.01 & 101.917 & 2.814 & 3 \\
\hline normal & 6.25 & $: 89.66$ & 0.003 & 0.438 & 0.007 & 191.07 & 3.85 & 4 \\
\hline normal & 7.25 & 89.639 & 0.002 & 0.429 & 0.005 & 385.462 & 5.456 & 6 \\
\hline normal & 8.25 & 89.645 & 0.002 & 0.422 & 0.003 & 786.729 & 7.816 & 9 \\
\hline normal & 9.25 & 89.663 & 0.001 & 0.429 & 0.003 & 1129.824 & 9.197 & 10 \\
\hline normal & 10.25 & 89.701 & 0.001 & 0.427 & 0.002 & 2204.774 & 12.765 & 15 \\
\hline normal & 11.25 & 89.743 & 0.001 & 0.435 & 0.001 & 3373.743 & 15.587 & 18 \\
\hline normal & 0.25 & 89.592 & 0.022 & 0.238 & 0.052 & 5.468 & 1.008 & 1 \\
\hline normal & 7.25 & 89.637 & 0.015 & $0: 404$ & 0.034 & 10.279 & 0.984 & 1 \\
\hline normal & 2.25 & 89.621 & 0.01 & 0.378 & 0.023 & 22.851 & 1.492 & 1.5 \\
\hline normal & 3.25 & 89.619 & 0.008 & 0.369 & 0.018 & 30.152 & 1.707 & 1.5 \\
\hline normal & 4.25 & 89.618 & 0.006 & 0.379 & 0.012 & 56.315 & 2.248 & 2 \\
\hline normal & 5.25 & 89.616 & 0.005 & 0.417 & 0.01 & 99.332 & 2.835 & 3 \\
\hline normal & 6.25 & 89.614 & 0.003 & 0.395 & 0.007 & 190.864 & 4.032 & 4 \\
\hline normal & 7.25 & 89.619 & 0.002 & 0.402 & 0.005 & 384.52 & 5.611 & 6 \\
\hline normal & 8.25 & 89.622 & 0.001 & 0.4 & 0.003 & 801.075 & 8.021 & 9 \\
\hline normal & 9.25 & 89.623 & 0.001 & 0.395 & 0.002 & 1207.104 & 9.858 & 10 \\
\hline normal & 10.25 & 89.627 & 0.001 & 0.393 & 0.002 & 2388.922 & 13.799 & 15 \\
\hline normal & 11.25 & 89.629 & 0.001 & 0.4 & 0.001 & 3755.341 & 17.073 & 18 \\
\hline normal & 678\% 64 & 89.606 & 0.003 & 0.4 & 0.006 & 203.025 & 1. 4127 & 4 \\
\hline normal & $68 \% 60$ & 89.614 & 0.003 & 0.39 & 0.006 & 204.638 & 17. 4.189 & 04 \\
\hline normal & $5 \quad-56$ & 89.596 & 0.003 & 0.406 & 0.006 & 205.456 & 4.105 & 4 \\
\hline normal & 1478652 & 89.58 & 0.003 & 0.392 & 0.006 & 203.314 & 0.4 .184 & 124 \\
\hline normal & $17 \times 48$ & 89.573 & 0.003 & 0.409 & 0.007 & 196.018 & 4.016 & 178 4 \\
\hline normal & 167844 & 89.564 & 0.003 & 0.404 & 0.006 & 204.323 & 4.127 & $\begin{array}{rl}4714 & 4 \\
\end{array}$ \\
\hline normal & $148-40$ & 89.563 & 0.003 & 0.392 & 0.006 & 209.179 & 4.243 & 4 \\
\hline normal & 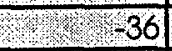 & 89.573 & 0.003 & 0.397 & 0.006 & 210992 & 44209 & $\begin{array}{l}7 \\
\end{array}$ \\
\hline normal & 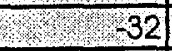 & 89.571 & 0.003 & (1) 0.4 & 0.006 & 212.3 & 4.216 & $\begin{array}{lll}7 & 4 \\
\end{array}$ \\
\hline normal & 28 & 89.579 & 0.003 & 0.391 & 0.006 & 217886 & 4.307 & 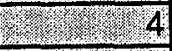 \\
\hline normal & 24 & 89.578 & 0.003 & 0.414 & 0.007 & 199.387 & 4.035 & 4 \\
\hline normal & (7) 20 & 89.581 & 0.003 & 0.393 & 0.006 & 213.472 & 48.247 & $\begin{array}{lll}72 & 4 \\
\end{array}$ \\
\hline normal & 16 & 89.594 & 0.003 & 0.417 & 0.006 & 206.56 & 4.046 & 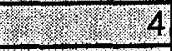 \\
\hline normal & (1) 14 & 89.606 & 0.003 & 0.409 & 0.006 & 203.57 & 4.092 & 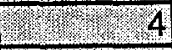 \\
\hline normal & -12 & 89.609 & 0.003 & 0.427 & 100006 & 203.602 & 3.971 & \begin{tabular}{|l|l|} 
& 4 \\
\end{tabular} \\
\hline
\end{tabular}




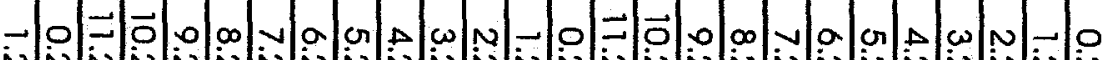

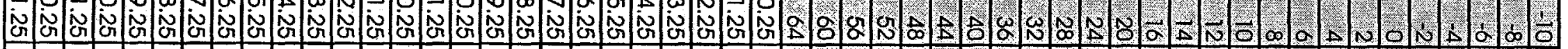
:

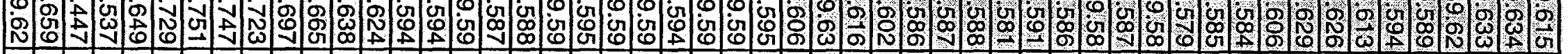

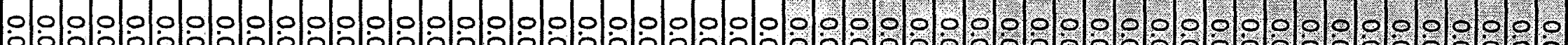

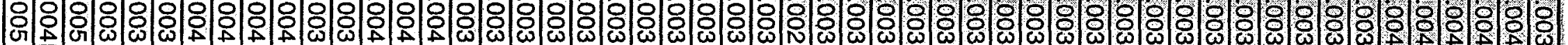

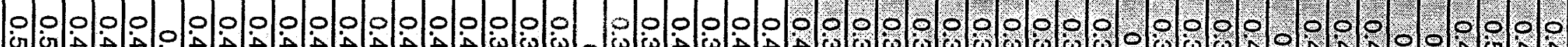

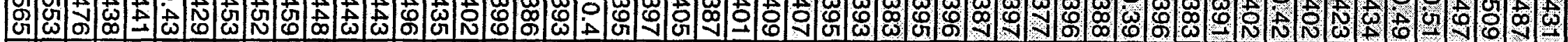

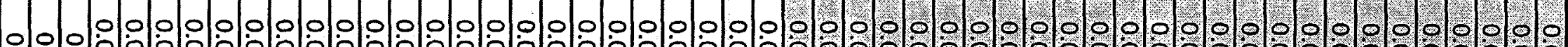

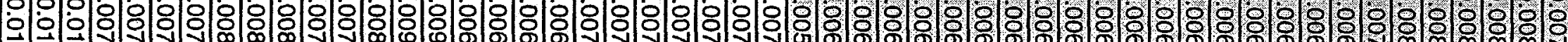

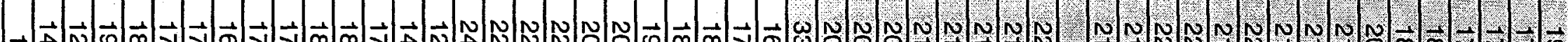

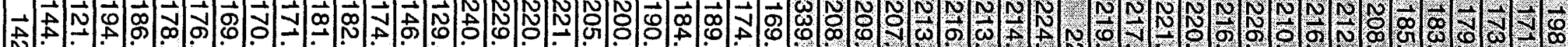

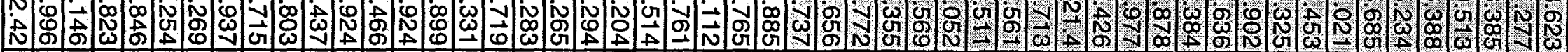

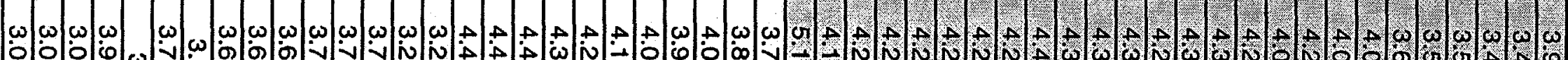

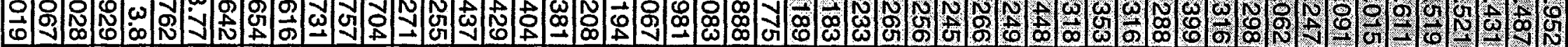

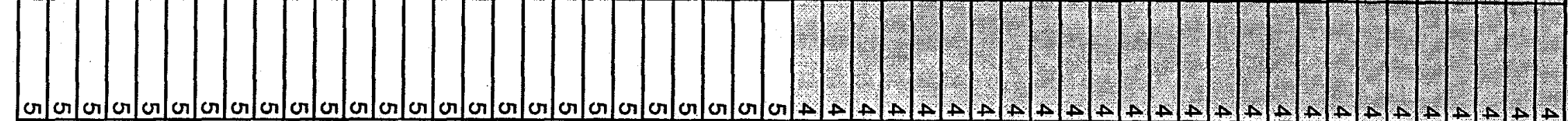




\begin{tabular}{|c|c|c|c|c|c|c|c|c|}
\hline transverse & 2.25 & 89.619 & 0.004 & 0.511 & 0.009 & 147.902 & 3.245 & 5 \\
\hline transverse & 3.25 & 89.647 & 0.004 & 0.491 & 0.009 & 155.836 & 3.368 & 5 \\
\hline transverse & 4.25 & 89.715 & 0.004 & 0.519 & 0.009 & $148: 563$ & 3.192 & 5 \\
\hline transverse & 5.25 & 89.796 & 0.004 & 0.532 & 0.01 & 148.664 & 3.178 & 5 \\
\hline transverse & 6.25 & 89.858 & 0.004 & 0.544 & 0.01 & 148.093 & 3.146 & 5 \\
\hline transverse & 7.25 & 89.878 & 0.004 & 0.519 & 0.009 & 155.295 & 3.283 & 5 \\
\hline transverse & 8.25 & 89.793 & 0.004 & 0.514 & 0.009 & 164.093 & 3.378 & 5 \\
\hline transverse & 9.25 & 89.62 & 0.004 & 0.497 & 0.008 & 178.323 & 3.536 & 5 \\
\hline transverse & 10.25 & 89.485 & 0.004 & 0.467 & 0.008 & 176.541 & 3.607 & 5 \\
\hline transverse & 11.25 & 89.414 & 0.005 & 0.47 & 0.01 & 116.089 & 2.978 & 5 \\
\hline transverse & 0.25 & 89.697 & 0.006 & 0.689 & 0.014 & 103.429 & 2.374 & 5 \\
\hline transverse & 1.25 & 89.636 & 0.005 & 0.594 & 0.011 & 125.176 & 2.783 & 5 \\
\hline transverse & 2.25 & 89.548 & 0.004 & 0.525 & 0.009 & 151.19 & 3.2 & 5 \\
\hline transverse & 3.25 & 89.579 & 0.004 & 0.522 & 0.009 & 155.829 & 3.248 & 5 \\
\hline transverse & 4.25 & 89.705 & 0.004 & 0.545 & 0.009 & 160.193 & 3.227 & 5 \\
\hline transverse & 5.25 & 89.818 & 0.004 & 0.53 & 0.009 & 167.803 & 3.333 & 5 \\
\hline transverse & 6.25 & 89.896 & 0.004 & 0.521 & 0.008 & 176.568 & 3.423 & 5 \\
\hline transverse & 7.25 & 89.917 & 0.004 & 0.523 & 0.008 & 181.503 & 3.472 & 5 \\
\hline transverse & 8.25 & 89.837 & 0.004 & 0.552 & 0.008 & 180.071 & 3.36 & 5 \\
\hline transverse & 9.25 & 89.632 & 0.004 & 0.536 & 0.008 & 184.468 & 3.46 & 5 \\
\hline transverse & 10.25 & 89.496 & 0.003 & 0.489 & 0.007 & 197.87 & 3.711 & 5 \\
\hline transverse & 11.25 & 89.443 & 0.005 & 0.494 & 0.01 & 120.239 & 2.982 & 5 \\
\hline transverse & 0.25 & 89.651 & 0.007 & 0.765 & 0.016 & 90.898 & 2.139 & 5 \\
\hline transverse & 1.25 & 89.644 & 0.007 & 0.714 & 0.015 & 96.624 & 2.316 & 5 \\
\hline transverse & 2.25 & 89.56 & 0.004 & 0.486 & 0.01 & 142.143 & 3.313 & 5 \\
\hline transverse & 3.25 & 89.539 & 0.004 & 0.479 & 0.008 & 161.167 & 3.439 & 5 \\
\hline transverse & 4.25 & 89.672 & 0.004 & 0.529 & 0.01 & 146.532 & 3.147 & 5 \\
\hline transverse & 5.25 & 89.786 & 0.004 & 0.499 & 0.009 & 164.141 & 3.429 & 5 \\
\hline transverse & 6.25 & 89.827 & 0.004 & 0.489 & 0.008 & 174.341 & 3.544 & 5 \\
\hline transverse & 7.25 & 89.838 & 0.003 & 0.474 & 0.007 & 193.594 & 3.767 & 5 \\
\hline transverse & 8.25 & 89.79 & 0.003 & 0.467 & 0.007 & 198.872 & 3.852 & 5 \\
\hline transverse & 9.25 & 89.664 & 0.003 & 0.47 & 0.007 & 206.572 & 3.892 & 5 \\
\hline transverse & 10.25 & 89.55 & 0.003 & 0.435 & 0.007 & 220.062 & 4.138 & 5 \\
\hline transverse & 11.25 & 89.464 & 0.004 & 0.461 & 0.008 & 158.026 & 3.444 & 5 \\
\hline transverse & 0.25 & 89.638 & 0.003 & 0.392 & 0.007 & 180.357 & 3.95 & 5 \\
\hline transverse & 7.25 & 89.619 & 0.003 & 0.403 & 0.007 & 178.833 & 3.891 & 5 \\
\hline transverse & 2.25 & 89.626 & 0.004 & 0.429 & 0.008 & 172.629 & 3.736 & 5 \\
\hline transverse & 3.25 & 89.648 & 0.004 & 0.445 & 0.008 & 169.376 & 3.654 & 5 \\
\hline transverse & 4.25 & 89.687 & 0.004 & 0.448 & 0.008 & 173.897 & 3.675 & 5 \\
\hline transverse & 5.25 & 89.713 & 0.003 & 0.444 & 0.007 & 184.358 & 3.814 & 5 \\
\hline transverse & 6.25 & 89.736 & 0.003 & 0.425 & 0.007 & 199.01 & 4.015 & 5 \\
\hline transverse & 7.25 & 89.737 & 0.003 & 0.428 & 0.007 & 204.191 & 4.029 & 5 \\
\hline transverse & 8.25 & 89.72 & 0.003 & 0.412 & 0.006 & 216.713 & 4.237 & 5 \\
\hline transverse & 9.25 & 89.654 & 0.003 & 0.413 & 0.006 & 229.06 & 4.331 & 5 \\
\hline transverse & 10.25 & 89.583 & 0.003 & 0.397 & 0.006 & 239.425 & 4.487 & 5 \\
\hline transverse & 11.25 & 89.507 & 0.004 & 0.418 & 0.008 & 165.248 & 3.709 & 5 \\
\hline transverse & 0.25 & 89.611 & 0.003 & 0.385 & 0.007 & 184.782 & 4.036 & 5 \\
\hline transverse & 1.25 & 89.607 & 0.003 & 0.387 & 0.007 & 190.999 & 4.071 & 5 \\
\hline transverse & 2.25 & 89.605 & 0.003 & 0.404 & 0.007 & 184.595 & 3.939 & 5 \\
\hline transverse & 3.25 & 89.606 & 0.003 & 0.389 & 0.007 & 196.089 & 4.172 & 5 \\
\hline transverse & 4.25 & 89.606 & 0.003 & 0.398 & 0.007 & 200.722 & 4.142 & 5 \\
\hline transverse & 5.25 & 89.611 & 0.003 & 0.39 & 0.006 & 212.411 & 4.321 & 5 \\
\hline
\end{tabular}




\begin{tabular}{|c|c|c|c|c|c|c|c|c|}
\hline transverse & 6.25 & 89.606 & 0.003 & 0.39 & 0.006 & 208.971 & 4.296 & 5 \\
\hline transverse & 7.25 & 89.602 & 0.003 & 0.395 & 0.006 & 214.36 & 4.29 & 5 \\
\hline transverse & 8.25 & 89.606 & 0.003 & 0.396 & 0.006 & 232.221 & 4.438 & 5 \\
\hline transverse & $9: 25$ & 89.601 & 0.003 & 0.401 & 0.006 & 230.269 & 4.392 & 5 \\
\hline transverse & 10.25 & 89.601 & 0.003 & 0.392 & 0.006 & 236.577 & 4.474 & 5 \\
\hline transverse & 17.25 & 89.618 & 0.004 & 0.445 & 0.008 & 154.11 & 3.496 & 5 \\
\hline transverse & $20-64$ & 89.641 & 0.003 & 0.383 & 0.006 & 204.165 & 4.282 & 5 \\
\hline transverse & 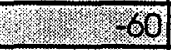 & 89.637 & 0.003 & 0.401 & 0.007 & 199.658 & 4.146 & 5 \\
\hline transverse & 1006 & 89.623 & 0.003 & 0.402 & 0.007 & 205.495 & 4.185 & 5 \\
\hline transverse & -52 & 89.607 & 0.003 & 0.389 & 0.006 & 205.132 & 4.244 & 5 \\
\hline transverse & 2. -48 & 89.602 & 0.003 & 0.414 & 0.007 & 196.006 & 3.992 & 5 \\
\hline transverse & $8 \quad 3 \quad-44$ & 89.587 & 0.003 & 0.409 & 0.007 & 198.514 & 4.085 & 5 \\
\hline transverse & $10-40$ & 89.59 & 0.003 & 0.384 & 0.007 & 203.195 & 4.263 & 5 \\
\hline transverse & $12-36$ & 89.588 & 0.003 & 0.388 & 0.006 & 204.589 & 4.269 & 5 \\
\hline transverse & -32 & 89.595 & 0.003 & 0.395 & 0.007 & 197.556 & 4.168 & 5 \\
\hline transverse & 28 & 89.599 & 0.003 & 0.402 & 0.007 & 201.173 & 4.15 & 5 \\
\hline transverse & 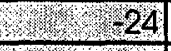 & 89.629 & 0.003 & 0.383 & 0.006 & 203.454 & 4.262 & 5 \\
\hline transverse & 20 & 89.657 & 0.003 & 0.405 & 0.007 & 194.913 & 4.072 & 5 \\
\hline transverse & $37-16$ & 89.681 & 0.003 & 0.424 & 0.007 & 183.924 & 3.886 & 5 \\
\hline transverse & 5.874 & 89.703 & 0.003 & 0.411 & 0.007 & 189.159 & 4.028 & 5 \\
\hline transverse & . $807 / 2$ & 89.716 & 0.003 & 0.43 & 0.007 & 180.471 & 3.835 & 5 \\
\hline transverse & $4878,-10$ & 89.75 & 0.004 & 0.472 & 0.009 & 158.915 & 3.465 & 5 \\
\hline transverse & 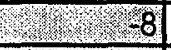 & 89.788 & 0.005 & 0.535 & 0.01 & 138.877 & 3.065 & 5 \\
\hline transverse & 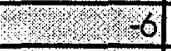 & 89.809 & 0.004 & 0.537 & 0.009 & 157.505 & 3.21 & 5 \\
\hline transverse & 178 & 89.827 & 0.004 & 0.506 & 0.009 & 164.96 & 3.395 & 5 \\
\hline transverse & $\sqrt{8} \times 4 \times 2$ & 89.821 & 0.004 & 0.53 & 0.009 & 164.885 & 3.299 & 5 \\
\hline transverse & 3rive & 89.805 & 0.004 & 0.515 & 0.009 & 158.569 & 3.316 & 5 \\
\hline transverse & 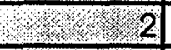 & 89.762 & 0.004 & 0.491 & 0.008 & 172.032 & 3.503 & 5 \\
\hline transverse & 48 & 89.725 & 0.003 & 0.446 & 0.007 & 187.495 & 3.823 & 5 \\
\hline transverse & $187 \% 6$ & 89.699 & 0.003 & 0.425 & 0.007 & 197.523 & 4.001 & 5 \\
\hline transverse & $\begin{array}{r}87 \\
\end{array}$ & 89.685 & 0.003 & 0.393 & 0.007 & 205.682 & 4.249 & 5 \\
\hline transverse & $10 \quad 10$ & 89.654 & 0.003 & 0.403 & 0.007 & 206.985 & 4.185 & 5 \\
\hline transverse & $10 \times 012$ & 89.629 & 0.003 & 0.386 & 0.007 & 207.971 & 4.307 & 5 \\
\hline transverse & 40014 & 89.606 & 0.003 & 0.377 & 0.007 & 204.473 & 4.358 & 5 \\
\hline transverse & 16 & 89.602 & 0.003 & 0.4 & 0.007 & 202.03 & 4.163 & 5 \\
\hline transverse & $3 \quad 20$ & 89.601 & 0.003 & 0.388 & 0.006 & 201.049 & 4.19 & 5 \\
\hline transverse & $2 \quad 24$ & 89.594 & 0.003 & 0.403 & 0.007 & 201.544 & 4.136 & 5 \\
\hline transverse & 9828 & 89.61 & 0.003 & 0.391 & 0.007 & 206.239 & 4.248 & 5 \\
\hline transverse & $\begin{array}{r}32 \\
\end{array}$ & 89.604 & 0.003 & 0.384 & 0.006 & 201.149 & 4.225 & 5 \\
\hline transverse & 370 & 89.606 & 0.003 & 0.402 & 0.007 & 199.558 & 4.149 & 5 \\
\hline transverse & $3 \quad 40$ & 89.605 & 0.003 & 0.405 & 0.007 & 199.69 & 4.124 & 5 \\
\hline transverse & 3744 & 89.605 & 0.003 & 0.404 & 0.007 & 203.164 & 4.139 & 5 \\
\hline transverse & 48 & 89.617 & 0.003 & 0.407 & 0.007 & 204.913 & 4.168 & 5 \\
\hline transverse & $1376 \quad 52$ & 89.635 & 0.003 & 0.389 & 0.006 & 210.35 & 4.296 & 5 \\
\hline transverse & 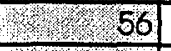 & 89.65 & 0.003 & 0.386 & 0.006 & 211.092 & 4.331 & 5 \\
\hline transverse & $4 \quad 60$ & 89.657 & 0.003 & 0.382 & 0.007 & 201.785 & 4.273 & 5 \\
\hline transverse & 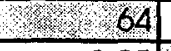 & 89.676 & 0.003 & 0.402 & 0.005 & 279.437 & 4.821 & 5 \\
\hline longitudina & 0.25 & 89768 & 0.003 & 0.369 & 0.007 & 240.695 & 5.25 & 20 \\
\hline longitudina & 1.25 & 89.728 & 0.002 & 0.354 & 0.005 & 37075 & 6.344 & $17 \times 20$ \\
\hline longitudina & 2.25 & 89719 & 0.002 & 0.365 & 0.005 & 356.191 & 6.199 & 20 \\
\hline longitudina & 3.25 & 89.713 & 0.003 & 0.357 & 0.005 & 343.931 & 6.165 & 20 \\
\hline longitudina & 4.25 & 89.713 & 0.003 & 0.36 & 0.006 & 338.761 & 6.076 & $\begin{array}{r}20 \\
\end{array}$ \\
\hline
\end{tabular}




\begin{tabular}{|c|c|c|c|c|c|c|c|c|}
\hline longitudina & 5.25 & 89.709 & 0.003 & 0.355 & 0.006 & 335.451 & 6.125 & (n) \\
\hline longitudina & 6.25 & 89.711 & 0.003 & 0.358 & 0.006 & 339.623 & 6.137 & 20 \\
\hline longitudina & 7.25 & 89.704 & 0.003 & 0.369 & 0.006 & 324091 & 5.928 & 20 \\
\hline Iongitudina & 8,25 & 89.710 & 0.003 & 0.36 & 0.006 & 347166 & 6.199 & 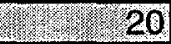 \\
\hline longitudina & 9.25 & 89.706 & 0.002 & 0.354 & 0.005 & 361.593 & 6.335 & 20 \\
\hline Iongitudina & 10.25 & 89.707 & 0.002 & 0,364 & 0.005 & 370.253 & 6.251 & 20 \\
\hline longitudina & 11.25 & 89704 & 0.002 & 0.371 & 0.005 & 3611.359 & 6.168 & 20 \\
\hline longitudina & 0.25 & 89.534 & 0.003 & 0.391 & 0.007 & 226.63 & 4.865 & 18 \\
\hline longitudina & 7.25 & 89.519 & 0.003 & 0.383 & 0.006 & 293.05 & 5.417 & 18 \\
\hline longitudina & 2.25 & 89.523 & 0.003 & 0.377 & 0.006 & 286.709 & 5.456 & 18 \\
\hline longitudina & 3.25 & 89.525 & 0.003 & 0.376 & 0.007 & 274.422 & 5.379 & 18 \\
\hline longituding & 4.25 & 89.525 & 0.003 & 0.37 & 0.006 & 280.421 & 5.502 & 18 \\
\hline longitudina & 5.25 & 89.517 & 0.003 & 0.387 & 0.007 & 271.851 & 5.293 & 18 \\
\hline longitudina & 6.25 & 89.502 & 0.003 & 0.404 & 0.007 & 259.106 & 5.073 & 18 \\
\hline Iongitudina & 7.25 & 89.484 & 0.003 & 0.401 & 0.007 & 269.8 & 5.186 & 18 \\
\hline longitudina & 8.25 & 89.475 & 0.003 & 0.391 & 0.007 & 272.325 & 5.254 & 18 \\
\hline longitudina & 9.25 & 89.480 & 0.003 & 0.409 & 0.007 & 264.191 & 5.093 & 18 \\
\hline longitudina & 10.25 & 89.483 & 0.003 & 0.415 & 0.007 & 264.732 & 5.061 & 18 \\
\hline longitudina & 11.25 & 89.492 & 0.003 & 0.432 & 0.008 & 247.237 & 4.854 & 18 \\
\hline longitudina & 0.25 & 89.511 & 0.003 & 0.403 & 0.008 & 223.564 & 4.776 & 1078 \\
\hline longitudina & 1.25 & 89.490 & 0.003 & 0.399 & 10006 & 293.69 & 5.336 & 6718 18 \\
\hline longitudina & 2.25 & 89.481 & 0.003 & 0.417 & 0.007 & 253.285 & 4.951 & $\begin{array}{l}18 \\
\end{array}$ \\
\hline longitudina & 3.25 & 89.465 & 0.004 & 0.446 & 0.008 & 234.352 & 4.678 & 1078 \\
\hline longitudina & 4.25 & 89.435 & 0.004 & 00,47 & 0.009 & 219.873 & 4.457 & $107 \% 18$ \\
\hline longitudina & 5.25 & 89.408 & 0.004 & 0.459 & 0.008 & 234.377 & $4 \quad 4.592$ & 28 \\
\hline longitudina & 6.25 & 89.377 & 0.003 & 0.424 & 0.008 & 246.964 & 4.891 & $\begin{array}{ll}18 \\
\end{array}$ \\
\hline longitudina & 7.25 & 89.362 & 0.004 & 0.453 & 0.008 & 236.027 & 4.593 & $8 \quad 18$ \\
\hline Tongit & 8.25 & 89.360 & 0.004 & 0.464 & 00.009 & 224.843 & 4.496 & 17tror 18 \\
\hline longitudina & 9.25 & 89.352 & 0.004 & 0.529 & 0.01 & 206.529 & 4.075 & 18 \\
\hline Iongitudina & 10.25 & 89.341 & 0.004 & 0.565 & 0.01 & 202,433 & 3.923 & 18 \\
\hline longitudina & 11.25 & 89.347 & 0.004 & 0.542 & 0.01 & 203.013 & 4.003 & 18 \\
\hline Iongitudina & 0.25 & 89.484 & 0.004 & 0.424 & 0.009 & 180.289 & 4.28 & 18 \\
\hline longitudina & 1.25 & 89.463 & 0.003 & 0.433 & 0.008 & 245.154 & 4.769 & 18 \\
\hline longitudina & 2.25 & 89.446 & 0.003 & 0.43 & 0.008 & 235.629 & 4.705 & 18 \\
\hline longituding & 3.25 & 89.436 & 0.004 & 0.457 & 0.008 & 221.815 & 4.488 & 18 \\
\hline Iongitudina & 4.25 & 89.429 & 0.004 & 0.477 & 0.009 & 217.862 & 4.378 & 18 \\
\hline longitudina & 5.25 & 89.410 & 0.003 & 0.435 & 0.008 & 242.358 & 4.752 & 18 \\
\hline longitudina & 6.25 & 89.373 & 0.003 & 0.427 & 0.008 & 246.691 & 4.865 & 18 \\
\hline longitudina & 7.25 & 89.332 & 0.003 & 0.394 & 0.007 & 262.469 & 5.19 & 18 \\
\hline longituding & 8.25 & 89.306 & 0.003 & 0.433 & 0.008 & 235.826 & 4.711 & 18 \\
\hline longitudina & 9.25 & 89.313 & 0.004 & 0.461 & 0.009 & 210.765 & 4.393 & 18 \\
\hline longitudina & 10.25 & 89.358 & 0.005 & 0.56 & 0.011 & 187.793 & 3.82 & 18 \\
\hline longitudina & 11.25 & 89.405 & 0.005 & 0.591 & 0.011 & 186.897 & 3.71 & 18 \\
\hline longi & 0.25 & 89785 & 0.004 & 0.341 & 0.008 & 183.55 & 4.874 & 18 \\
\hline longitudina & 1.25 & 89721 & 0.0 .02 & 0.341 & 0.005 & 333825 & 6.127 & 18 \\
\hline longitu & 2.25 & 89.699 & 0.003 & 0.353 & 0.006 & 331.566 & 6.044 & 18 \\
\hline longituding & 3.25 & 89.698 & 0.003 & 0.354 & 0.006 & 311867 & 5.859 & 18 \\
\hline longitudina & 4.25 & 89.697 & 0.003 & 0.346 & 0.006 & 305,492 & 5.887 & 18 \\
\hline longitudina & 5.25 & 89.697 & 0.003 & 0.359 & 0.006 & 298,419 & 5.749 & $\begin{array}{r}18 \\
\end{array}$ \\
\hline longitudina & 6.25 & 89.698 & 0.003 & 0.347 & 0.006 & 308.672 & 5.961 & 18 \\
\hline longitudina & 7.25 & 89.692 & 0.003 & 0.337 & 0.006 & 317.177 & 6.124 & 10.18 \\
\hline longitudina & 8.25 & 89.701 & 0.003 & 0.367 & 0.006 & $306: 414$ & 5.771 & 1 18 \\
\hline
\end{tabular}




\begin{tabular}{|c|c|c|c|c|c|c|c|c|}
\hline longitudina & 9.25 & 89.697 & 0.003 & 0.353 & 0.005 & 329.998 & 5.998 & 18 \\
\hline longitudina & 10.25 & 89.700 & 0.003 & 0.351 & 0.005 & 333.577 & 6.063 & 18 \\
\hline longituding & 11.25 & 89.696 & 0.002 & 0.356 & 80.005 & 338.948 & 2.6 .023 & 18018 \\
\hline Iongitudina & 0.25 & 89.582 & 0.005 & 0.345 & 0.011 & 127.059 & 4.258 & 18 \\
\hline longitudina & 1.25 & 89.534 & 0.003 & 0.364 & 0.006 & 279.771 & 5.458 & 18 \\
\hline Iongitudina & 2.25 & 89.525 & 0.003 & 0.366 & 0.006 & 305.094 & 5.67 & 18 \\
\hline Iongitudina & 3.25 & 89.528 & 0.003 & 0.36 & 0.006 & 309.95 & 5.759 & 18 \\
\hline longituding & 4.25 & 89.525 & 0.003 & 0.355 & 0.006 & 297.904 & 5.77 & 18 \\
\hline longitudina & 5.25 & 89.525 & 0.003 & 0.356 & 0.006 & 295.87 & 5.708 & 18 \\
\hline longitudina & 6.25 & 89.515 & 0.003 & 0.372 & 0.006 & 289.133 & 5.527 & 18 \\
\hline longitudina & 7.25 & 89.501 & 0.003 & 0.349 & 0.006 & 300.997 & 5.808 & 18 \\
\hline longitudina & 8.25 & 89.486 & 0.003 & 0.371 & 0.006 & 297.298 & 5.583 & 18 \\
\hline longitudina & 9.25 & 89.469 & 0.003 & 0.362 & 0.006 & 312.996 & 5.763 & 18 \\
\hline longituding & 10.25 & 89.464 & 0.003 & 0.356 & 0.006 & 301.363 & 5.726 & 18 \\
\hline longitudina & 17.25 & 89.464 & 0.003 & 0.375 & 0.006 & 307.576 & 5.609 & 18 \\
\hline longituding & 0.25 & 89.525 & 0.005 & 0.368 & 0.012 & 117.745 & 4.017 & 18 \\
\hline longitudina & 1.25 & 89.493 & 0.003 & 0.394 & 0.007 & 272.78 & 3. 5.19 & 2018 \\
\hline Iongitudina & 2.25 & 89494 & 0.003 & 0.41 & 0.007 & 278.226 & 5.137 & 18 \\
\hline longitudina & 3.25 & 89.501 & 0.003 & 0.386 & 0.006 & 281.531 & 5.343 & 148: 18 \\
\hline longitudina & 4.25 & 89.495 & 0.003 & 0.378 & 0.007 & 270.57 & 5.322 & 2018 \\
\hline longitudina & 5.25 & 89.481 & 0.003 & 0.378 & 0.007 & 268.881 & 5.342 & $2 \times 2 x=18$ \\
\hline longitudina & 6.25 & 89.467 & 0.003 & 0.39 & 0.007 & 262163 & 5206 & X717\%18 \\
\hline longitudina & 7.25 & 89.426 & 0.003 & 0.398 & 0.007 & 2607 & 5129 & 18 \\
\hline longitudina & 8.25 & 89.410 & 0.003 & 00.4 & 0.007 & 262.111 & 5.105 & 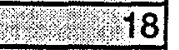 \\
\hline longitudina & 9.25 & 89.413 & 0.003 & 0.405 & 0.0007 & 245.023 & 4.94 & 818 \\
\hline Iongitudina & 10.25 & 89.438 & 0.004 & 0.496 & 0.009 & 202.881 & 4.211 & 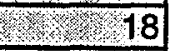 \\
\hline Iongitudina & 11.25 & 89.452 & 0.005 & 0.568 & 0.012 & 179.643 & 3.823 & 818 \\
\hline Iongitudina & 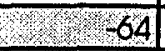 & 89.614 & 0.003 & 0.372 & 0.006 & 280.693 & 5.485 & 18 \\
\hline Iongitudina & 83760 & 89.621 & 0.003 & 0.377 & 0.006 & 273.869 & 5.378 & 18 \\
\hline longitudina & 18\% 56 & 89.602 & 0.003 & 0.376 & 0.006 & 280.304 & 5.452 & 18 \\
\hline longitudina & $8 \% \quad 52$ & 89.588 & 0.003 & 0.368 & 0.006 & 280.581 & 5.507 & 18 \\
\hline longitudina & 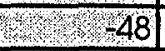 & 89.609 & 0.003 & 0.353 & 0.006 & 289.285 & 5.696 & 18 \\
\hline Iongitudina & 767844 & 89.655 & 0.003 & 0.371 & 0.006 & 293.965 & 5.627 & 18 \\
\hline longitudina & 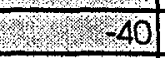 & 89.708 & 0.003 & 0.362 & 0.006 & 297.641 & 5.702 & 18 \\
\hline longitudina & -36 & 89.746 & 0.003 & 0.364 & 0.006 & 289.011 & 5.66 & 18 \\
\hline longitudina & 17. 32 & 89.762 & 0.003 & 0.355 & 0.006 & 311.738 & 5.934 & 18 \\
\hline longitudina & $13 \times 428$ & 89.761 & 0.003 & 0.365 & 0.006 & 297.485 & 5.72 & 18 \\
\hline longitudina & $1:-24$ & 89.760 & 0.003 & 0.352 & 0.006 & 284.531 & 5.725 & 18 \\
\hline longitudina & $3 \quad 820$ & 89.745 & 0.003 & 0.362 & 0.006 & 292.374 & 5.723 & 18 \\
\hline Iding & $30-76$ & 89.707 & 0.003 & 0.355 & 0.006 & 297.553 & 5.808 & 18 \\
\hline longit & 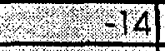 & 89.639 & 0.003 & 0.381 & 0.007 & 268.323 & 5.323 & 18 \\
\hline longit & $8 \quad-12$ & 89.568 & 0.003 & 0.38 & 0.007 & 276.474 & 5.388 & 18 \\
\hline longitudina & $48-10$ & 89.542 & 0.003 & 0.37 & 0.006 & 283.952 & 5.519 & 18 \\
\hline longitudina & 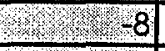 & 89.506 & 0.003 & 0.395 & 0.007 & 259.599 & 5.146 & 18 \\
\hline longitudina & 06 & 89.427 & 0.003 & 0.435 & 0.008 & 238.656 & 4.737 & 18 \\
\hline longitudina & 48 & 89.371 & 0.003 & 0.423 & 0.007 & 249.83 & 4.907 & 18 \\
\hline longitudina & $10-2$ & 89.358 & 0.003 & 0.421 & 0.007 & 265.007 & 5.011 & 18 \\
\hline longitudind & 300 & 89.374 & 0.003 & 0.41 & 0.007 & 256.102 & 5.005 & 18 \\
\hline longitudina & 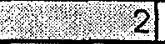 & 89.379 & 0.003 & 0.43 & 0.008 & 239.821 & 4.788 & 18 \\
\hline longitudina & 4 & 89.470 & 0.003 & 0.372 & 0.007 & 269.772 & 5.357 & 18 \\
\hline longitudina & 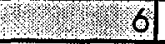 & 89.500 & 0.003 & 0.364 & 0.006 & 286.898 & 5.546 & 18 \\
\hline longitudina & 8 & 89.530 & 0.003 & 0.359 & 0.006 & 304.819 & 5.75 & 18 \\
\hline
\end{tabular}




\begin{tabular}{|l|r|r|r|r|r|r|r|r|}
\hline longitudina & 10 & 89.565 & 0.003 & 0.364 & 0.006 & 290.608 & 5.621 & 18 \\
\hline longitudina & 12 & 89.610 & 0.003 & 0.354 & 0.006 & 293.856 & 5.706 & 18 \\
\hline longitudina & 14 & 89.656 & 0.003 & 0.348 & 0.006 & 298.207 & 5.823 & 18 \\
\hline longitudina & 16 & 89.682 & 0.003 & 0.366 & 0.006 & 302.534 & 5.737 & 18 \\
\hline longitudina & 20 & 89.713 & 0.003 & 0.351 & 0.006 & 301.502 & 5.825 & 18 \\
\hline longitudina & 24 & 89.715 & 0.003 & 0.356 & 0.006 & 300.878 & 5.819 & 18 \\
\hline longitudina & 28 & 89.712 & 0.003 & 0.355 & 0.006 & 300.133 & 5.838 & 18 \\
\hline longitudina & 32 & 89.702 & 0.003 & 0.357 & 0.006 & 304.136 & 5.827 & 18 \\
\hline longitudina & 36 & 89.707 & 0.003 & 0.348 & 0.006 & 309.941 & 5.979 & 18 \\
\hline longitudina & 40 & 89.703 & 0.003 & 0.351 & 0.006 & 312.766 & 5.918 & 18 \\
\hline longitudina & 44 & 89.697 & 0.003 & 0.358 & 0.006 & 306.325 & 5.816 & 18 \\
\hline longitudina & 48 & 89.688 & 0.003 & 0.357 & 0.006 & 305.623 & 5.81 & 18 \\
\hline longitudina & 52 & 89.685 & 0.003 & 0.341 & 0.006 & 314.527 & 6.005 & 18 \\
\hline longitudina & 56 & 89.671 & 0.003 & 0.37 & 0.006 & 294.687 & 5.593 & 18 \\
\hline longitudina & 60 & 89.621 & 0.003 & 0.37 & 0.006 & 302.293 & 5.675 & 18 \\
\hline longitudina & 64 & 89.578 & 0.003 & 0.362 & 0.006 & 292.253 & 5.61 & 18 \\
\hline
\end{tabular}


Ferritic Steel DO Comb-like Sample

\begin{tabular}{|c|c|c|c|c|c|c|c|c|}
\hline direction & $\begin{array}{r}\text { Position } \\
(\mathbf{m m})\end{array}$ & $\begin{array}{c}2 \Theta / d / a \\
{[\circ / A]}\end{array}$ & uncertainty & $\begin{array}{c}\text { FWHM } \\
{\left[{ }^{\circ}\right]}\end{array}$ & $\begin{array}{c}\text { uncertainty } \\
{\left[{ }^{\circ}\right]}\end{array}$ & \begin{tabular}{|c|}
$\begin{array}{c}\text { Peak } \\
\text { Height }\end{array}$ \\
\end{tabular} & uncertainty & $\begin{array}{c}\text { sampling } \\
\text { time (min.) }\end{array}$ \\
\hline normal & 0.25 & 89.613 & 0.006 & 0.403 & 0.015 & 132.2 & 4.718 & 50 \\
\hline normal & 1.25 & 89.627 & 0.005 & 0.377 & 0.012 & 142.55 & 4.433 & 30 \\
\hline normal & 2.25 & 89.628 & 0.006 & 0.425 & 0.015 & 85.443 & 3.104 & 15 \\
\hline normal & 3.25 & 89.62 & 0.005 & 0.371 & 0.011 & 110.71 & 3.541 & 12 \\
\hline normal & 4.25 & 89.623 & 0.005 & 0.409 & 0.01 & 115.98 & 3.337 & 10 \\
\hline normal & 5.25 & 89.623 & 0.004 & 0.385 & 0.009 & 130.03 & 3.52 & 8 \\
\hline normal & 6.25 & 89.63 & 0.003 & 0.4 & 0.007 & 182.76 & 4.048 & 9 \\
\hline normal & 7.25 & 89.624 & 0.003 & 0.396 & 0.006 & 255.43 & \begin{tabular}{|l|}
4.717 \\
\end{tabular} & 10 \\
\hline normal & 8.25 & 89.628 & 0.002 & 0.406 & 0.005 & 351.49 & 5.448 & 12 \\
\hline normal & 9.25 & 89.62 & 0.002 & 0.406 & 0.004 & 553.62 & 6.756 & 15 \\
\hline normal & 10.25 & 89.618 & 0.001 & 0.396 & 0.002 & \begin{tabular}{|l|}
1442.1 \\
\end{tabular} & 10.871 & 30 \\
\hline normal & 11.25 & 89.626 & 0.001 & 0.402 & 0.002 & 2922.3 & 15.315 & 50 \\
\hline normal & 0.25 & 89.617 & 0.008 & 0.703 & 0.019 & 146.48 & 3.737 & 50 \\
\hline normal & 1.25 & 89.633 & 0.006 & 0.556 & 0.015 & 134.92 & 3.667 & 30 \\
\hline normal & 2.25 & 89.61 & 0.006 & 0.458 & 0.013 & 110.65 & 3.313 & 15 \\
\hline normal & 3.25 & 89.624 & 0.005 & 0.466 & 0.012 & 108.45 & 3.161 & 12 \\
\hline normal & 4.25 & 89.625 & 0.005 & 0.454 & 0.01 & 128.88 & 3.334 & 10 \\
\hline normal & 5.25 & 89.63 & 0.004 & 0.442 & \begin{tabular}{l|l}
0.009 \\
\end{tabular} & 132.41 & 3.34 & 8 \\
\hline normal & 6.25 & 89.634 & 0.003 & 0.426 & 0.008 & 186.89 & 3.97 & 9 \\
\hline normal & 7.25 & 89.64 & 0.003 & 0.443 & 0.007 & 237.99 & 4.331 & 10 \\
\hline normal & 8.25 & 89.639 & 0.002 & 0.429 & 0.005 & \begin{tabular}{|l|}
357.17 \\
\end{tabular} & 5.353 & 12 \\
\hline normal & 9.25 & 89.636 & 0.002 & 0.444 & 0.004 & 537.51 & 6.414 & 15 \\
\hline normal & 10.25 & 89.638 & 0.001 & 0.44 & 0.002 & 1428.3 & 10.322 & 30 \\
\hline normal & 11.25 & 89.639 & 0.001 & 0.448 & 0.002 & 2651 & 13.901 & 50 \\
\hline normal & 0.25 & 89.636 & 0.005 & 0.561 & \begin{tabular}{l|}
0.012 \\
\end{tabular} & 215.84 & 4.572 & 50 \\
\hline normal & 1.25 & 89.629 & 0.005 & 0.578 & 0.012 & 179.76 & 3.871 & 30 \\
\hline norma! & 2.25 & 89.609 & 0.006 & 0.561 & 0.014 & 109.81 & 2.973 & 15 \\
\hline normal & 3.25 & 89.634 & 0.005 & 0.505 & 0.012 & 118.59 & 3.114 & 12 \\
\hline normal & 4.25 & 89.613 & 0.004 & 0.453 & 0.01 & 134.85 & 3.371 & 10 \\
\hline normal & 5.25 & 89.628 & 0.005 & 0.522 & 0.011 & 122.23 & 2.965 & 8 \\
\hline normal & 6.25 & 89.632 & 0.004 & 0.513 & 0.009 & 168.65 & 3.469 & 9 \\
\hline normal & 7.25 & 89.647 & 0.004 & 0.536 & 0.008 & \begin{tabular}{|l|}
201.56 \\
\end{tabular} & 3.689 & 10 \\
\hline normal & 8.25 & 89.653 & 0.003 & 0.499 & 0.006 & 315.42 & 4.707 & 12 \\
\hline normal & 9.25 & 89.634 & 0.002 & 0.488 & 0.005 & 501.95 & 5.92 & 15 \\
\hline normal & 10.25 & 89.643 & 0.001 & 0.462 & 0.003 & 1338.5 & 9.808 & 30 \\
\hline normal & 11.25 & 89.636 & 0.001 & 0.469 & 0.002 & \begin{tabular}{|r|}
2434 \\
\end{tabular} & 13.102 & 50 \\
\hline normal & 0.25 & 89.629 & 0.006 & 0.72 & 0.014 & 218.71 & 4.102 & 50 \\
\hline normal & 1.25 & 89.618 & 0.005 & 0.639 & 0.013 & \begin{tabular}{|l|}
179.83 \\
\end{tabular} & 3.717 & 30 \\
\hline normal & 2.25 & 89.624 & 0.005 & 0.518 & 0.012 & 137.6 & 3.391 & 15 \\
\hline normal & 3.25 & 89.619 & 0.004 & 0.473 & 0.01 & 154.58 & 3.569 & 12 \\
\hline normal & 4.25 & 89.627 & 0.005 & 0.512 & 0.011 & 126.44 & 3.124 & 10 \\
\hline normal & 5.25 & 89.632 & 0.005 & 0.502 & 0.01 & \begin{tabular}{|l|}
138.2 \\
\end{tabular} & 3.21 & 9 \\
\hline normal & 6.25 & 89.635 & 0.004 & 0.493 & 0.009 & 154.36 & 3.387 & 8 \\
\hline normal & 7.25 & 89.639 & 0.005 & 0.508 & 0.01 & 129.36 & 3.009 & 6 \\
\hline normal & 8.25 & 89.633 & 0.005 & 0.534 & 0.011 & 106.67 & 2.636 & 4 \\
\hline normal & 9.25 & 89.615 & 0.004 & 0.509 & 0.01 & $\mid 117.71$ & 2.806 & 3.5 \\
\hline normal & 10.25 & 89.639 & 0.004 & 0.481 & 0.009 & 130.74 & 3.023 & 3 \\
\hline
\end{tabular}




\begin{tabular}{|c|c|c|c|c|c|c|c|c|}
\hline normal & 11.25 & 89.624 & 0.004 & 0.472 & 0.008 & 132.05 & 3.037 & 3 \\
\hline normal & 0.25 & 89.61 & 0.008 & 0.792 & 0.019 & 154.448 & 3.539 & 50 \\
\hline normal & 7.25 & 89.618 & 0.007 & 0.667 & 0.017 & 135.136 & 3.333 & 30 \\
\hline normal & 2.25 & 89.618 & 0.005 & 0.516 & 0.013 & 123.655 & 3.243 & 15 \\
\hline normal & 3.25 & 89.633 & 0.005 & 0.481 & 0.012 & 120.498 & 3.216 & 12 \\
\hline normal & 4.25 & 89.64 & 0.005 & 0.502 & 0.011 & 124.552 & 3.12 & 10 \\
\hline normal & 5.25 & 89.631 & 0.004 & 0.465 & 0.009 & 152.543 & 3.507 & 9 \\
\hline normal & 6.25 & 89.626 & 0.004 & 0.482 & 0.008 & 173.398 & 3.561 & 8 \\
\hline normal & 7.25 & 89.637 & 0.004 & 0.497 & 0.009 & 142.793 & 3.185 & 6 \\
\hline normal & 8.25 & 89.63 & 0.004 & 0.454 & 0.009 & 115.815 & 2.968 & 4 \\
\hline normal & 9.25 & 89.634 & 0.004 & 0.433 & 0.008 & 141.909 & 3.323 & 3.5 \\
\hline normal & 10.25 & 89.639 & 0.004 & 0.452 & 0.007 & 152.839 & 3.309 & 3 \\
\hline normal & 17.25 & 89.636 & 0.004 & 0.465 & 0.008 & 129.356 & 3.02 & 3 \\
\hline normal & 0.25 & 89.629 & 0.004 & 0.414 & 0.009 & 260.418 & 5.618 & 50 \\
\hline normal & 1.25 & 89.621 & 0.004 & 0.436 & 0.009 & 215.826 & 4.739 & 30 \\
\hline normal & 2.25 & 89.615 & 0.004 & 0.426 & 0.01 & 150.061 & 3.82 & 15 \\
\hline normal & 3.25 & 89.64 & 0.004 & 0.431 & 0.009 & 148.344 & 3.645 & 12 \\
\hline normal & 4.25 & 89.639 & 0.004 & 0.415 & 0.009 & 156.212 & 3.769 & 10 \\
\hline normal & 5.25 & 89.631 & 0.004 & 0.422 & 0.008 & 162.521 & 3.767 & 9 \\
\hline normal & 6.25 & 89.629 & 0.003 & 0.418 & 0.007 & 191.123 & 4.009 & 8 \\
\hline normal & 7.25 & 89.635 & 0.004 & 0.414 & 0.007 & 164.472 & 3.68 & 6 \\
\hline normal & 8.25 & 89.64 & 0.004 & 0.433 & 0.008 & 125.406 & 3.119 & 4 \\
\hline normal & 9.25 & 89.626 & 0.004 & 0.421 & 0.008 & 141.024 & 3.37 & 3.5 \\
\hline normal & 10.25 & 89.626 & 0.003 & 0.412 & 0.007 & 158.569 & 3.521 & 3 \\
\hline normal & 11.25 & 89.632 & 0.004 & 0.45 & 0.008 & 143.411 & 3.235 & 3 \\
\hline normal & 0.25 & 89.62 & 0.006 & 0.411 & 0.014 & 148.481 & 4.841 & 50 \\
\hline normal & 1.25 & 89.629 & 0.005 & 0.406 & 0.012 & 142.919 & 4.302 & 30 \\
\hline normal & 2.25 & 89.623 & 0.005 & 0.379 & 0.012 & 105.611 & 3.548 & 15 \\
\hline normal & 3.25 & 89.62 & 0.005 & 0.405 & 0.011 & 109.326 & 3.362 & 12 \\
\hline normal & 4.25 & 89.637 & 0.005 & 0.419 & 0.01 & 120.228 & 3.348 & 10 \\
\hline normal & 5.25 & 89.632 & 0.004 & 0.415 & 0.009 & 147.078 & 3.601 & 9 \\
\hline normal & 6.25 & 89.629 & 0.004 & 0.408 & 0.008 & 166.607 & 3.814 & 8 \\
\hline normal & 7.25 & 89.63 & 0.004 & 0.399 & 0.007 & 158.788 & 3.702 & 6 \\
\hline normal & 8.25 & 89.627 & 0.004 & 0.388 & 0.008 & 135.058 & 3.409 & 4 \\
\hline normal & 9.25 & 89.618 & 0.004 & 0.397 & 0.007 & 142.032 & 3.421 & 3.5 \\
\hline normal & 10.25 & 89.616 & 0.003 & 0.394 & 0.007 & 147.689 & 3.491 & 3 \\
\hline normal & 17.25 & 89.634 & 0.003 & 0.417 & 0.006 & 182.317 & 3.729 & 3 \\
\hline
\end{tabular}




\section{Detector \#5 \\ Ferritic Steel Weld Plate}

\begin{tabular}{|c|c|c|c|c|c|c|c|c|}
\hline direction & $\begin{array}{c}\text { Position } \\
(\mathrm{mm})\end{array}$ & $\begin{array}{c}2 \Theta / d / a \\
{[0 / A]}\end{array}$ & uncertainty & $\begin{array}{c}\text { FWHM } \\
{\left[{ }^{\circ}\right]}\end{array}$ & $\begin{array}{c}\text { uncertainty } \\
{\left[{ }^{\circ}\right]}\end{array}$ & \begin{tabular}{|c|} 
Peak \\
Height
\end{tabular} & uncertainty & $\begin{array}{c}\text { sampling } \\
\text { time }\end{array}$ \\
\hline normal & 0.25 & \begin{tabular}{|l|}
89.507 \\
\end{tabular} & 0.008 & 0.465 & \begin{tabular}{|r|}
0.019 \\
\end{tabular} & 77.063 & 3.097 & Den? 18 \\
\hline normal & 1.25 & 89.518 & 0.005 & 0.406 & 0.011 & 126.38 & 3.744 & 15 \\
\hline normal & 2.25 & 89.52 & 0.005 & 0.433 & 0.011 & 117.98 & 3.348 & 10 \\
\hline normal & 3.25 & 89.524 & 0.004 & 0.425 & 0.009 & 148.63 & 3.646 & 9 \\
\hline normal & 4.25 & 89.532 & 0.004 & 0.427 & 0.009 & 146.61 & 3.498 & 6 \\
\hline normal & 5.25 & 89.534 & 0.005 & 0.416 & 0.01 & 98.466 & 2.872 & 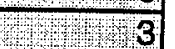 \\
\hline normal & 6.25 & 89.542 & 0.003 & 0.417 & 0.007 & 176.57 & 3.795 & $x=4$ \\
\hline normal & 7.25 & 89.538 & 0.002 & 0.413 & 0.005 & 372.25 & 5.403 & 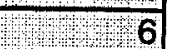 \\
\hline normal & 8.25 & 89.541 & 0.002 & 0.409 & 0.003 & \begin{tabular}{|l|}
763.76 \\
\end{tabular} & 7.719 & 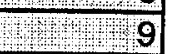 \\
\hline normal & 9.25 & 89.546 & 0.001 & 0.409 & 0.003 & 1128.6 & 9.289 & 10 \\
\hline normal & 10.25 & 89.548 & 0.001 & 0.408 & 0.002 & \begin{tabular}{|l|}
2230.3 \\
\end{tabular} & 12.968 & 15 \\
\hline normal & 11.25 & 89.546 & 0.001 & 0.412 & 0.001 & 3433.5 & 15.942 & 18 \\
\hline normal & 0.25 & 89.591 & 0.008 & 0.459 & 0.019 & 79.775 & 3.181 & 18 \\
\hline normal & 1.25 & 89.599 & 0.005 & 0.422 & 0.011 & 134.87 & 3.768 & 15 \\
\hline normal & 2.25 & 89.607 & 0.005 & 0.454 & 0.011 & 123.73 & 3.349 & 10 \\
\hline normal & 3.25 & 89.6 & 0.004 & 0.431 & 0.009 & 154.31 & 3.742 & 9 \\
\hline normal & 4.25 & 89.591 & 0.004 & 0.427 & 0.009 & 141.67 & 3.47 & 6 \\
\hline norrval & 5.25 & 89.583 & 0.005 & 0.423 & 0.01 & 101.25 & 2.865 & 3 \\
\hline normal & 6.25 & 89.566 & 0.003 & 0.427 & 0.007 & 180.49 & 3.761 & 4 \\
\hline normal & 7.25 & 89.565 & 0.002 & 0.426 & 0.005 & 367.57 & 5.349 & 6 \\
\hline normal & 8.25 & 89.568 & 0.002 & 0.418 & 0.003 & \begin{tabular}{|l|l|}
744.43 \\
\end{tabular} & 7.607 & 9 \\
\hline normal & 9.25 & 89.588 & 0.001 & 0.42 & 0.003 & \begin{tabular}{|l|}
1097.3 \\
\end{tabular} & 9.1 & 10 \\
\hline normal & 10.25 & 89.614 & 0.001 & 0.417 & 0.002 & 2141.1 & 12.681 & 15 \\
\hline normal & 11.25 & 89.644 & 0.001 & 0.418 & 0.001 & 3423 & 15.942 & 18 \\
\hline normal & 0.25 & 89.635 & 0.01 & 0.6 & 0.025 & 67.281 & 2.673 & 18 \\
\hline normal & 1.25 & 89.652 & 0.007 & 0.608 & 0.018 & 99.198 & 2.88 & 15 \\
\hline normal & 2.25 & 89.66 & 0.008 & 0.692 & 0.019 & 86.317 & 2.421 & 10 \\
\hline normal & 3.25 & 89.66 & 0.006 & 0.524 & 0.013 & 116.28 & 3.11 & 9 \\
\hline normal & 4.25 & 89.66 & 0.004 & 0.464 & 0.009 & 138.21 & 3.325 & 6 \\
\hline normal & 5.25 & 89.626 & 0.005 & 0.472 & 0.012 & 91.542 & 2.64 & ME: \\
\hline normal & 6.25 & 89.597 & 0.004 & 0.482 & 0.008 & 163.69 & 3.443 & 4 \\
\hline nornal & 7.25 & 89.572 & 0.003 & 0.489 & 0.006 & 330.85 & 4.747 & 6 \\
\hline normal & 8.25 & 89.572 & 0.002 & 0.465 & 0.004 & 675.23 & 6.894 & 9 \\
\hline normal & 9.25 & 89.595 & 0.001 & 0.453 & 0.003 & 1028.2 & 8.532 & 10 \\
\hline normal & 10.25 & 89.642 & 0.001 & 0.448 & 0.002 & 2001.6 & 11.898 & $14 \div 15$ \\
\hline normal & 11.25 & 89.679 & 0.001 & 0.441 & 0.002 & 3139.2 & 14.968 & 18 \\
\hline normal & 0.25 & 89.557 & 0.009 & 0.746 & 0.022 & 89.467 & 2.585 & 18 \\
\hline normal & 1.25 & 89.598 & 0.008 & 0.654 & 0.017 & 103.18 & 2.797 & 15 \\
\hline normal & 2.25 & 89.649 & 0.006 & 0.543 & 0.014 & 109.79 & 2.973 & 10 \\
\hline normal & 3.25 & 89.673 & 0.004 & 0.483 & 0.009 & 167.38 & 3.662 & 9 \\
\hline normal & 4.25 & 89.656 & 0.004 & 0.493 & 0.01 & 145.39 & 3.327 & $\frac{7}{6}$ \\
\hline normal & 5.25 & 89.63 & 0.006 & 0.513 & 0.012 & 89.273 & 2.509 & 3 \\
\hline normal & 6.25 & 89.581 & 0.004 & 0.498 & 0.008 & 164.04 & 3.396 & 4 \\
\hline normal & 7.25 & 89.562 & 0.003 & 0.535 & 0.006 & 306.58 & 4.412 & 6 \\
\hline normal & 8.25 & 89.563 & 0.002 & 0.548 & 0.004 & 593.47 & 6.033 & 9 \\
\hline normal & 9.25 & 89.613 & 0.002 & 0.52 & 0.003 & 900.88 & 7.521 & 10 \\
\hline normal & 10.25 & 89.667 & 0.001 & 0.471 & 0.002 & \begin{tabular}{|l|}
1929.7 \\
\end{tabular} & 11.488 & 15 \\
\hline
\end{tabular}




\begin{tabular}{|c|c|c|c|c|c|c|c|c|}
\hline normal & 11.25 & 89.699 & 0.001 & 0.464 & 0.002 & 2979.434 & 14.272 & 18 \\
\hline normal & 0.25 & 89.544 & 0.01 & 0.74 & 0.024 & 86.294 & 2.606 & 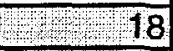 \\
\hline normal & 1.25 & 89.557 & 0.008 & 0.701 & 0.019 & 99.046 & 2.696 & $=1$ \\
\hline normal & 2.25 & 89.626 & 0.008 & 0.66 & 0.019 & 88.969 & 2.533 & 10 \\
\hline normal & 3.25 & 89.658 & 0.005 & 0.51 & 0.011 & 143.044 & 3.351 & 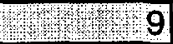 \\
\hline normal & 4.25 & 89.668 & 0.004 & 0.476 & 0.01 & 141.817 & 3.332 & $=$ \\
\hline normal & 5.25 & 89.601 & 0.006 & 0.529 & 0.012 & 90.174 & 2.463 & 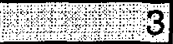 \\
\hline normal & 6.25 & 89.54 & 0.004 & 0.514 & 0.008 & 165.156 & 3.329 & 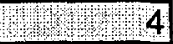 \\
\hline normal & 7.25 & 89.527 & 0.003 & 0.515 & 0.006 & 329.343 & 4.645 & 6 \\
\hline normal & 8.25 & 89.53 & 0.002 & 0.52 & 0.004 & 627.875 & 6.331 & $=$ \\
\hline normal & 9.25 & 89.58 & 0.002 & 0.488 & 0.003 & 955.383 & 7.985 & 10 \\
\hline normal & 10.25 & 89.642 & 0.001 & 0.465 & 0.002 & 1983.007 & 11.685 & E: \\
\hline normal & 11.25 & 89.684 & 0.001 & 0.461 & 0.002 & 3123.606 & 14.618 & nim \\
\hline normal & 0.25 & 89.541 & 0.007 & 0.477 & 0.017 & 92.839 & 3.272 & 18 \\
\hline normal & 1.25 & 89.57 & 0.005 & 0.463 & 0.012 & 128.617 & 3.566 & 15 \\
\hline normal & 2.25 & 89.598 & 0.005 & 0.421 & 0.01 & 135.259 & 3.617 & 10 \\
\hline normal & 3.25 & 89.636 & 0.004 & 0.447 & 0.009 & 159.036 & 3.727 & 9 \\
\hline normal & 4.25 & 89.635 & 0.004 & 0.431 & 0.009 & 155.166 & 3.637 & 6 \\
\hline normal & 5.25 & 89.605 & 0.005 & 0.433 & 0.01 & 103.578 & 2.868 & 3 \\
\hline normal & 6.25 & 89.588 & 0.003 & 0.455 & 0.007 & 182.677 & 3.685 & 4 \\
\hline normal & 7.25 & 89.57 & 0.002 & 0.44 & 0.005 & 368.903 & 5.258 & 6 \\
\hline normal & 8.25 & 89.566 & 0.002 & 0.436 & 0.003 & 735.111 & 7.398 & $\overline{9}$ \\
\hline normal & 9.25 & 89.586 & 0.001 & 0.436 & 0.003 & 1070.866 & 8.837 & 10 \\
\hline normal & 10.25 & 89.616 & 0.001 & 0.433 & 0.002 & 2110.795 & 12.381 & 15 \\
\hline normal & 11.25 & 89.654 & 0.001 & 0.435 & 0.002 & 3205.347 & 15.158 & 18 \\
\hline normal & 0.25 & 89.459 & 0.022 & 0.379 & 0.056 & 5.998 & 0.842 & 1 \\
\hline normal & 1.25 & 89.494 & 0.03 & 0.327 & 0.086 & 6.166 & 1.152 & 1 \\
\hline normal & 2.25 & 89.555 & 0.013 & 0.448 & 0.028 & 16.918 & 1.184 & 1.5 \\
\hline normal & 3.25 & 89.539 & 0.009 & 0.409 & 0.021 & 27.189 & 1.549 & 1.5 \\
\hline normal & 4.25 & 89.535 & 0.006 & 0.395 & 0.013 & 51.553 & 2.103 & 2 \\
\hline normal & 5.25 & 89.532 & 0.005 & 0.406 & 0.01 & 100.088 & 2.909 & 3 \\
\hline normal & 6.25 & 89.54 & 0.003 & 0.425 & 0.007 & 179.911 & 3.762 & 4 \\
\hline normal & 7.25 & 89.545 & 0.002 & 0.414 & 0.005 & 371.876 & 5.416 & 6 \\
\hline normal & 8.25 & 89.545 & 0.002 & 0.405 & 0.003 & 774.595 & 7.798 & 9 \\
\hline normal & 9.25 & 89.55 & 0.001 & 0.406 & 0.002 & 1145.453 & 9.377 & 10 \\
\hline normal & 10.25 & 89.547 & 0.001 & 0.405 & 0.002 & 2269.379 & 13.136 & 15 \\
\hline normal & 11.25 & 89.544 & 0.001 & 0.41 & 0.001 & 3608.364 & 16.342 & 18 \\
\hline normal &.-64 & 89.53 & 0.003 & 0.409 & 0.007 & 197.379 & 4.007 & 4 \\
\hline normal & -60 & 89.535 & 0.003 & 0.417 & 0.007 & 202.595 & $3: 996$ & 1) \\
\hline normal & -56 & 89.515 & 0.003 & 0.417 & 0.006 & 210 & 4.086 & 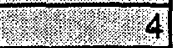 \\
\hline normal & -52 & 89.514 & 0.003 & 0.415 & 0.007 & 208.735 & 4.069 & $\cos 2 x$ \\
\hline normal & -48 & 89.5 & 0.003 & 0.409 & 0.006 & 206.207 & 4.089 & 27) 4 \\
\hline normal & -44 & 89.491 & 0.003 & 0.4 & 0.006 & 213.311 & $2=4196$ & $3 x^{2}=4$ \\
\hline normal & -40 & 89.49 & 0.003 & 0.418 & 0.006 & 209.805 & 4.065 & 2 \\
\hline normal & -36 & 89.488 & 0.003 & 0.411 & 0.006 & 208.522 & 0408 & 4 \\
\hline normal & -32 & 89.502 & 0.003 & 0.395 & 0.006 & $211: 173$ & 4.4198 & 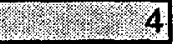 \\
\hline normal & -28 & 89.502 & 0.003 & 0.419 & 0.006 & 206.305 & 2.024 & 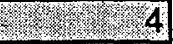 \\
\hline normal & $9-24$ & 89.497 & 0.003 & 0.399 & 0.006 & 213.167 & 4.191 & 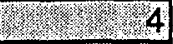 \\
\hline normal & 78:-20 & 89.506 & 0.003 & 0.42 & 0.006 & 207.682 & 4.036 & $12=4$ \\
\hline normal & -16 & 89.523 & 0.003 & 0.416 & 0.006 & 207.8 & 4.063 & $x=4$ \\
\hline normal & $14=-14$ & 89.53 & $0: 003$ & 0.411 & 0.007 & 199.355 & 4.02 & 4 \\
\hline normal & -12 & 89.535 & 0.003 & 0.436 & 0.007 & 196.149 & 3.873 & 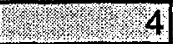 \\
\hline
\end{tabular}




\begin{tabular}{|c|c|c|c|c|c|c|c|c|}
\hline normal & 190 & 89.534 & 0.003 & 0.449 & 0.007 & 19482 & 3835 & \\
\hline normal & -8 & 89.555 & 0.004 & 0.503 & 0.008 & 168.263 & 3.41 & \\
\hline ormal & -6 & 89.553 & 0.004 & 0.527 & 0.008 & 182.824 & 3.46 & \\
\hline ormal & 30 & 89.538 & 0.004 & 0.512 & 0.008 & 181.818 & 3.477 & \\
\hline normal & - & 89.505 & 0.004 & 0.53 & 0008 & 183.675 & 3.449 & \\
\hline ormal & 0 & 89.513 & 0.004 & 0.518 & 0.008 & 185.546 & 3.508 & \\
\hline prmal & 2 & 89.539 & 0.003 & 0.447 & 0.007 & 197793 & 3.866 & \\
\hline ormal & 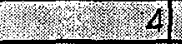 & 89.548 & 0.003 & 0.436 & 0.007 & 202.757 & 3.948 & \\
\hline normal & 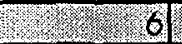 & 89.554 & 0.003 & 0.419 & 0.006 & 208.363 & 4.055 & \\
\hline prmal & 8 & 89.529 & 0.003 & 0.424 & 0.007 & 195.981 & 3.936 & \\
\hline rmal & 2.20 & 89.503 & $\begin{array}{r}0.003 \\
\end{array}$ & 0.415 & 0.006 & 216.007 & 4.123 & \\
\hline ormal & 1012 12 & 89.5 & 0.003 & 0.414 & 0.006 & 211.651 & 4.108 & \\
\hline rmal & 14 & 89.501 & 0.003 & 0.401 & 0.006 & 221.947 & 4.257 & \\
\hline ormal & 1016 & 89.502 & 0.003 & 0.415 & 0.006 & 211.832 & 4.091 & \\
\hline rmal & 1.:20 & 89.505 & 0.003 & 0.409 & 0.006 & 215.34 & 4.155 & \\
\hline normal & 24 & 89.504 & 0.003 & 0.42 & 0.006 & 205.688 & 4.001 & \\
\hline normal & 28 & 89.507 & 0.003 & 0.404 & 20.006 & 220.795 & 4.232 & . \\
\hline normal & $\quad 32$ & 89.506 & 0.003 & 0.406 & 0.006 & 214776 & 4.166 & $\operatorname{sen}$ \\
\hline normal & $\begin{array}{r}36 \\
\end{array}$ & 89.509 & 0.003 & 0.399 & 0.006 & 215.093 & 4.201 & 2 \\
\hline normal & $3=40$ & 89.505 & 0.003 & 0.411 & 0.006 & 205.828 & 4.054 & 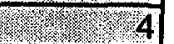 \\
\hline normal & 44 & 89.509 & 0.003 & 0.414 & 0.006 & 205.009 & 4.013 & 17?:20 \\
\hline normal & $\begin{array}{r}48 \\
\end{array}$ & 8951 & 0.003 & 0.411 & 0.006 & 202865 & 4.025 & 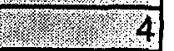 \\
\hline normal & $5 \% 52$ & 89.522 & 0.003 & 0.404 & 0.006 & 205078 & 4.096 & S \\
\hline normal & $\begin{array}{r}56 \\
\end{array}$ & 89.538 & 0.003 & 0.396 & 0.006 & 209.353 & 4.181 & y \\
\hline normal & 60 & 89.547 & 0.003 & 0.403 & 0.006 & $206: 483$ & 4.078 & 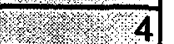 \\
\hline normal & 64 & 89.519 & 0.002 & 0.418 & 0.005 & 333.856 & 5.027 & \\
\hline transverse & 0.25 & 89.506 & 0.004 & 0.408 & 0.007 & 167.137 & 3.719 & $\overline{5}$ \\
\hline transverse & 1.25 & 89.505 & 0.003 & 0.408 & 0.007 & 183.787 & 3.889 & $\overline{5}$ \\
\hline transverse & 2.25 & 89.506 & 0.003 & 0.414 & 0.007 & 186.211 & 3.894 & $\overline{5}$ \\
\hline transverse & 3.25 & 89.508 & 0.003 & 0.407 & 0.007 & 191.275 & 3.991 & 5 \\
\hline transverse & 4.25 & 89.512 & 0.003 & 0.423 & 0.007 & 193.852 & 3.965 & $\overline{5}$ \\
\hline transverse & 5.25 & 89.508 & 0.003 & 0.413 & 0.007 & 205.235 & 4.102 & 5 \\
\hline transverse & 6.25 & 89.514 & 0.003 & 0.415 & 0.006 & 216.529 & 4.16 & 5 \\
\hline transverse & 7.25 & 89.513 & 0.003 & 0.406 & 0.006 & 226.086 & 4.294 & 5 \\
\hline transverse & 8.25 & 89.505 & 0.003 & 0.418 & 0.006 & 218.516 & 4.192 & 5 \\
\hline transverse & 9.25 & 89.507 & 0.003 & 0.419 & 0.006 & 223.227 & 4.19 & 5 \\
\hline insyerse & 10.25 & 89.503 & 0.003 & 0.408 & 0.006 & 231.611 & 4.307 & 5 \\
\hline insverse & 17.25 & 89.511 & 0.004 & 0.44 & 0.009 & 126.507 & 3.206 & 5 \\
\hline insverse & 0.25 & 89.528 & 0.004 & 0.516 & 0.009 & 144.781 & 3.177 & 5 \\
\hline ansverse & 1.25 & 89.539 & 0.004 & 0.455 & 0.008 & 174.527 & 3.635 & 5 \\
\hline ansverse & 2.25 & 89.561 & 0.003 & 0.446 & 0.007 & 185.957 & 3.764 & 5 \\
\hline transverse & 3.25 & 89.582 & 0.003 & 0.453 & 0.007 & 187.572 & 3.778 & 5 \\
\hline transverse & 4.25 & 89.616 & 0.004 & 0.455 & 0.008 & 178.564 & 3.707 & 5 \\
\hline transverse & 5.25 & 89.649 & 0.004 & 0.449 & 0.008 & 178.014 & 3.71 & 5 \\
\hline transverse & 6.25 & 89.656 & 0.004 & 0.454 & 0.008 & 179.973 & 3.735 & \\
\hline transverse & 7.25 & 89.671 & 0.004 & 0.447 & 0.008 & 179.234 & 3.736 & \\
\hline transverse & 8.25 & 89.646 & 0.004 & 0.44 & 0.007 & 179.704 & 3.745 & 5 \\
\hline transverse & 9.25 & 89.559 & 0.003 & 0.441 & 0.007 & 189.828 & 3.834 & \\
\hline transverse & 10.25 & 89.448 & 0.003 & 0.45 & 0.007 & 191.899 & 3.812 & 5 \\
\hline transverse & 11.25 & 89.36 & 0.004 & 0.465 & 0.009 & 131.622 & 3.182 & \\
\hline transverse & 0.25 & 89.58 & 0.005 & 0.568 & 0.01 & 146.732 & 3.032 & \\
\hline transverse & 1.25 & 89.533 & 0.004 & 0.559 & 0.01 & 151.407 & 3.109 & \\
\hline
\end{tabular}




\begin{tabular}{|c|c|c|c|c|c|c|c|c|}
\hline transverse & 2.25 & 89.534 & 0.004 & 0.541 & 0.009 & 152.885 & 3.172 & 5 \\
\hline transverse & 3.25 & 89.563 & 0.004 & 0.52 & 0.009 & 155.052 & 3.252 & 5 \\
\hline transverse & 4.25 & 89.617 & 0.004 & 0.523 & 0.009 & 148.792 & 3.187 & 5 \\
\hline transverse & 5.25 & 89.711 & 0.004 & 0.501 & 0.01 & 148.902 & 3.33 & 5 \\
\hline transverse & 6.25 & 89.779 & 0.004 & 0.528 & 0.009 & 156.561 & 3.272 & 5 \\
\hline transverse & 7.25 & 89.797 & 0.004 & 0.542 & 0.009 & 156.589 & 3.23 & 5 \\
\hline transverse & 8.25 & 89.708 & 0.004 & 0.512 & 0.009 & 172.849 & 3.466 & 5 \\
\hline transverse & 9.25 & 89.534 & 0.004 & 0.51 & 0.008 & 176.124 & 3.466 & 5 \\
\hline transverse & 10.25 & 89.388 & 0.003 & 0.458 & 0.007 & 190.988 & 3.786 & 5 \\
\hline transverse & 11.25 & 89.314 & 0.005 & 0.485 & 0.01 & 115.976 & 2.98 & 5 \\
\hline transverse & 0.25 & 89.608 & 0.006 & 0.697 & 0.014 & 106.647 & 2.397 & 5 \\
\hline transverse & 1.25 & 89.548 & 0.005 & 0.61 & 0.011 & 126.572 & 2.756 & 5 \\
\hline transverse & 2.25 & 89.455 & 0.004 & 0.524 & 0.009 & 154.447 & 3.22 & 5 \\
\hline transverse & 3.25 & 89.484 & 0.004 & 0.52 & 0.009 & 160.835 & 3.274 & 5 \\
\hline transverse & 4.25 & 89.615 & 0.004 & 0.549 & 0.01 & 150.583 & 3.13 & 5 \\
\hline transverse & 5.25 & 89.73 & 0.004 & 0.486 & 0.009 & 171.517 & 3.58 & 5 \\
\hline transverse & 6.25 & 89.804 & 0.004 & 0.528 & 0.008 & 179.818 & 3.469 & 5 \\
\hline transverse & 7.25 & 89.829 & 0.004 & 0.513 & 0.008 & 188.484 & 3.583 & 5 \\
\hline transverse & 8.25 & 89.742 & 0.004 & 0.532 & 0.008 & 182.969 & 3.469 & 5 \\
\hline transverse & 9.25 & 89.539 & 0.004 & 0.536 & 0.008 & 188.473 & 3.497 & 5 \\
\hline transverse & 10.25 & 89.404 & 0.003 & 0.476 & 0.007 & 205.901 & 3.818 & 5 \\
\hline transverse & 11.25 & 89.349 & 0.005 & 0.489 & 0.01 & 129.083 & 3.12 & 5 \\
\hline transverse & 0.25 & 89.576 & 0.007 & 0.778 & 0.016 & 95.766 & 2.177 & 5 \\
\hline transverse & 1.25 & 89.553 & 0.007 & 0.721 & 0.016 & 96.98 & 2.317 & 5 \\
\hline transverse & 2.25 & 89.463 & 0.005 & 0.518 & 0.01 & 140.994 & 3.199 & 5 \\
\hline transverse & 3.25 & 89.452 & 0.004 & 0.481 & 0.008 & 162.941 & 3.461 & 5 \\
\hline transverse & 4.25 & 89.591 & 0.004 & 0.519 & 0.01 & 148.44 & 3.225 & 5 \\
\hline transverse & 5.25 & 89.693 & 0.004 & 0.498 & 0.009 & 169.002 & 3.487 & 5 \\
\hline transverse & 6.25 & 89.742 & 0.004 & 0.485 & 0.008 & 181.217 & 3.621 & 5 \\
\hline transverse & 7.25 & 89.757 & 0.004 & 0.476 & 0.008 & 187.574 & 3.721 & 5 \\
\hline transverse & 8.25 & 89.7 & 0.003 & 0.46 & 0.007 & 205.253 & 3.942 & 5 \\
\hline transverse & 9.25 & 89.58 & 0.003 & 0.445 & 0.007 & 210.831 & 4.03 & 5 \\
\hline transverse & 10.25 & 89.462 & 0.003 & 0.452 & 0.007 & 217.703 & 4.024 & 5 \\
\hline transverse & 71.25 & 89.378 & 0.004 & 0.469 & 0.009 & 158.213 & 3.462 & 5 \\
\hline transverse & 0.25 & 89.549 & 0.003 & 0.41 & 0.007 & 175.682 & 3.813 & 5 \\
\hline transverse & 1.25 & 89.527 & 0.004 & 0.428 & 0.007 & 171.956 & 3.692 & 5 \\
\hline transverse & 2.25 & 89.534 & 0.004 & 0.434 & 0.008 & 172.98 & 3.712 & 5 \\
\hline transverse & 3.25 & 89.558 & 0.003 & 0.434 & 0.007 & 183.57 & 3.815 & 5 \\
\hline transverse & 4.25 & 89.599 & 0.004 & 0.438 & 0.008 & 184.249 & 3.805 & 5 \\
\hline transverse & 5.25 & 89.633 & 0.003 & 0.439 & 0.007 & 199.283 & 3.987 & 5 \\
\hline transverse & 6.25 & 89.65 & 0.003 & 0.428 & 0.007 & 198.719 & 4.021 & 5 \\
\hline transverse & 7.25 & 89.651 & 0.003 & 0.428 & 0.007 & 209.725 & 4.124 & 5 \\
\hline transverse & 8.25 & 89.626 & 0.003 & 0.418 & 0.006 & 220.38 & 4.227 & 5 \\
\hline transverse & 9.25 & 89.572 & 0.003 & 0.419 & 0.006 & 221.558 & 4.2 & 5 \\
\hline transverse & 10.25 & 89.499 & 0.003 & 0.424 & 0.006 & 233.82 & 4.262 & 5 \\
\hline transverse & 11.25 & 89.432 & 0.004 & 0.438 & 0.008 & 163.546 & 3.608 & 5 \\
\hline transverse & 0.25 & 89.524 & 0.003 & 0.407 & 0.007 & 175.416 & 3.801 & 5 \\
\hline transverse & 1.25 & 89.527 & 0.003 & 0.397 & 0.007 & 189.149 & 4.036 & 5 \\
\hline transverse & 2.25 & 89.522 & 0.003 & 0.395 & 0.007 & 193.514 & 4.065 & 5 \\
\hline transverse & 3.25 & 89.518 & 0.003 & 0.404 & 0.007 & 195.307 & 4.041 & 5 \\
\hline transverse & 4.25 & 89.523 & 0.003 & 0.393 & 0.007 & 200.662 & 4.175 & 5 \\
\hline transverse & 5.25 & 89.524 & 0.003 & 0.411 & 0.007 & 200.755 & 4.05 & 5 \\
\hline
\end{tabular}




\begin{tabular}{|c|c|c|c|c|c|c|c|c|}
\hline transverse & 6.25 & 89.517 & 0.003 & 0.413 & 0.007 & 201.305 & 4.059 & 5 \\
\hline transverse & 7.25 & 89.516 & 0.003 & 0.41 & 0.006 & 219.751 & 4.242 & 5 \\
\hline transverse & 8.25 & 89.519 & 0.003 & 0.397 & 0.006 & 225.732 & 4.331 & 5 \\
\hline transverse & 9.25 & 89.517 & 0.003 & 0.401 & 0.006 & 237.382 & 4.411 & 5 \\
\hline transverse & 10.25 & 89.515 & 0.003 & 0.4 & 0.006 & 242.814 & 4.468 & 5 \\
\hline transverse & 11.25 & 89.527 & 0.004 & 0.447 & 0.008 & 159.201 & 3.499 & 5 \\
\hline transverse & 564 & 89.555 & 0.003 & 0.404 & 0.007 & 202.73 & 4.161 & 5 \\
\hline transverse & WV1 & 89.549 & 0.003 & 0.405 & 0.007 & 206.455 & 4.16 & 5 \\
\hline transverse & 10 -56 & 89.535 & 0.003 & 0.402 & 0.007 & 201.361 & 4.116 & 5 \\
\hline transverse & \begin{tabular}{|l|l|}
52 \\
\end{tabular} & 89.521 & 0.003 & 0.413 & 0.007 & 200.343 & 4.064 & 5 \\
\hline transverse & T/2 -48 & 89.511 & 0.003 & 0.404 & 0.007 & 200.093 & 4.063 & 5 \\
\hline transverse & 17.21 .44 & 89.497 & 0.003 & 0.405 & 0.007 & 199.455 & 4.087 & 5 \\
\hline transverse & (2) & 89.503 & 0.003 & 0.413 & 0.007 & 207.332 & 4.104 & 5 \\
\hline transverse & $3 \quad-36$ & 89.505 & 0.003 & 0.409 & 0.007 & 197.388 & 4.051 & 5 \\
\hline transverse & -32 & 89.512 & 0.003 & 0.395 & 0.007 & .196 .824 & 4.135 & 5 \\
\hline transverse & $3=-28$ & 89.515 & 0.003 & 0.418 & 0.007 & 199.221 & 4.017 & 5 \\
\hline transverse & - 24 & 89.548 & 0.003 & 0.408 & 0.007 & 195.266 & 4.03 & 5 \\
\hline transverse & 120 20 & 89.578 & 0.003 & 0.41 & 0.007 & 192.737 & 4.016 & 5 \\
\hline transverse & 16 & 89.604 & 0.003 & 0.411 & 0.007 & 202.267 & 4.083 & 5 \\
\hline transverse & 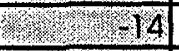 & 89.618 & 0.003 & 0.414 & 0.007 & 196.191 & 4.043 & 5 \\
\hline transverse & 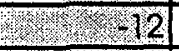 & 89.639 & 0.003 & 0.438 & 0.007 & 192.247 & 3.892 & 5 \\
\hline transverse & 7.10 & 89.671 & 0.004 & 0.487 & 0.009 & 159.595 & 3.434 & 5 \\
\hline transverse & 80 & 89.701 & 0.004 & 0.512 & 0.01 & 143.075 & 3.197 & 5 \\
\hline transverse & 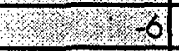 & 89.719 & 0.004 & 0.519 & 0.009 & 162.22 & 3.347 & 5 \\
\hline transverse & $\sqrt{186} \times 4$ & 89.735 & 0.004 & 0.498 & 0.008 & 172.514 & 3.508 & 5 \\
\hline transverse & 76. & 89.742 & 0.004 & 0.525 & 0.009 & 166.142 & 3.38 & 5 \\
\hline transverse & 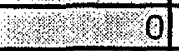 & 89.723 & 0.004 & 0.508 & 0.009 & 162.329 & 3.406 & 5 \\
\hline transverse & 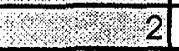 & 89.677 & 0.004 & 0.467 & 0.008 & 179.331 & 3.685 & 5 \\
\hline transverse & 4 & 89.644 & 0.003 & 0.443 & 0.007 & 188.321 & 3.848 & 5 \\
\hline transverse & 6 & 89.621 & 0.003 & 0.421 & 0.007 & 195.771 & 4 & 5 \\
\hline transverse & $8 \times 8$ & 89.602 & 0.003 & 0.403 & 0.007 & 205.028 & 4.184 & 5 \\
\hline transverse & 10 & 89.574 & 0.003 & 0.396 & 0.007 & 208.14 & 4.251 & 5 \\
\hline transverse & 12 & 89.544 & 0.003 & 0.404 & 0.007 & 199.444 & 4.125 & 5 \\
\hline transverse & $787 \times 14$ & 89.533 & 0.003 & 0.401 & 0.007 & 207.278 & 4.174 & 5 \\
\hline transverse & 18 & 89.519 & 0.003 & 0.409 & 0.007 & 199.349 & 4.109 & 5 \\
\hline transverse & 20 20 & 89.519 & 0.003 & 0.417 & 0.007 & 193.889 & 3.987 & 5 \\
\hline transverse & 24 & 89.516 & 0.003 & 0.409 & 0.007 & 201.75 & 4.092 & 5 \\
\hline transverse & 28 & 89.522 & 0.003 & 0.411 & 0.007 & 202.748 & 4.088 & 5 \\
\hline transverse & $\begin{array}{r}32 \\
\end{array}$ & 89.523 & 0.004 & 0.419 & 0.008 & 181.878 & 3.885 & 5 \\
\hline transverse: & 36 & 89.518 & 0.003 & 0.416 & 0.007 & 206.222 & 4.12 & 5 \\
\hline transverse & $1 \times 40$ & 89.523 & 0.003 & 0.4 & 0.007 & 202.774 & 4.166 & 5 \\
\hline transverse & $4 \quad 44$ & 89.521 & 0.003 & 0.407 & 0.007 & 203.131 & 4.104 & 5 \\
\hline transverse & 48 & 89.532 & 0.003 & 0.42 & 0.007 & 197.603 & 4.018 & 5 \\
\hline transverse & $\times 52$ & 89.542 & 0.003 & 0.392 & 0.007 & 202.664 & 4.201 & 5 \\
\hline transverse & 28.56 & 89.562 & 0.003 & 0.399 & 0.007 & 201.654 & 4.16 & 5 \\
\hline transverse & 60 & 89.582 & 0.003 & 0.412 & 0.007 & 192.354 & 4.011 & 5 \\
\hline transverse & $6 \quad 64$ & 89.598 & 0.003 & 0.413 & 0.005 & 285.836 & 4.806 & 5 \\
\hline longitudina & 0.25 & 89.674 & 0.004 & 0.404 & 0.008 & 217723 & 4.864 & 20 \\
\hline longitudina & 1.25 & 89.646 & 0.003 & 0.418 & 0.006 & 335.293 & 5.658 & $\begin{array}{l}20 \\
\end{array}$ \\
\hline longituding & 2.25 & 89.640 & 0.003 & 0.415 & 0.006 & 336.923 & 5.675 & $\begin{array}{r}70 \\
\end{array}$ \\
\hline Iongitudina & 3.25 & 89.629 & 0.003 & 0.42 & 0.006 & 314824 & 5.495 & $\begin{array}{r}20 \\
\end{array}$ \\
\hline longitudina & 4.25 & 89.632 & 0.003 & 0.411 & 0.006 & 314.696 & 5.56 & $\begin{array}{r}20 \\
\end{array}$ \\
\hline
\end{tabular}




\begin{tabular}{|c|c|c|c|c|c|c|c|c|}
\hline Jongitudind & 5.25 & 89.632 & 0.003 & 0.414 & 0.006 & 315.399 & 0725.554 & 20 \\
\hline longitudina & 6.25 & 89.619 & 0.003 & 0.403 & 0.006 & 311776 & 5.632 & 20 \\
\hline longitudina & 7.25 & 89.625 & 0.003 & 0.413 & 0.006 & 313.708 & 5.547 & 20 \\
\hline longitudina & 8.25 & 89.631 & 0.003 & 0.427 & 0.007 & 3044461 & 5.383 & 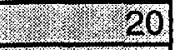 \\
\hline Iongitudina & 9.25 & 89.625 & 0.003 & 0.411 & 0.006 & 335.306 & 5.716 & 28 \\
\hline longitudina & 10.25 & 89621 & 0.003 & 0.409 & 0.006 & 340.589 & 5.724 & 20 \\
\hline Iongitudina & 11.25 & 89.621 & 0.003 & 0.412 & 0.006 & 342.11 & 5.728 & 20 \\
\hline longitudina & 0.25 & 89.435 & 0.004 & 0.456 & 0.009 & 202.979 & 4.304 & 18 \\
\hline longitudina & 1.25 & 89.433 & 0.003 & 0.454 & 0.007 & 270.169 & 4.826 & 18 \\
\hline longitudina & 2.25 & 89.456 & 0.004 & 0.469 & 0.008 & 239.524 & 4.545 & 18 \\
\hline longitudina & 3.25 & 89.447 & 0.003 & 0.453 & 0.008 & 249.433 & 4.699 & 18 \\
\hline longitudina & 4.25 & 89.451 & 0.003 & 0.448 & 0.008 & 245.881 & 4.738 & 18 \\
\hline Iongitudina & 5.25 & 89.440 & 0.004 & 0.473 & 0.008 & 240.431 & 4.568 & 18 \\
\hline longitudina & 6.25 & 89.429 & 0.004 & 0.489 & 0.009 & 220.983 & 4.33 & 18 \\
\hline Iongitudina & 7.25 & 89.420 & 0.004 & 0.497 & 0.009 & 225.93 & 4.344 & 18 \\
\hline longitudina & 8.25 & 89.396 & 0.004 & 0.493 & 0.009 & 228.334 & 4.366 & 18 \\
\hline longitudina & 9.25 & 89.407 & 0.004 & 0.511 & 0.009 & 223.441 & 4.21 & 18 \\
\hline longitudina & 10.25 & 89.423 & 0.004 & 0.526 & 0.009 & 227.978 & 4.237 & 18 \\
\hline longitudina & 11.25 & 89.433 & 0.004 & 0.524 & 0.009 & 219.264 & 4.185 & 18 \\
\hline Iongitudina & 0.25 & 89.412 & 0.004 & 0.491 & 0.01 & 187.688 & 4.012 & 18 \\
\hline longitudina & 1.25 & 89,412 & 0.004 & 0.507 & 0.008 & 235.704 & 4.326 & 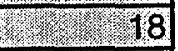 \\
\hline longitudina & 2.25 & 89.407 & 0,004 & 0.503 & 0.009 & 223.103 & 4255 & 18 \\
\hline longitudina & 3.25 & 89.395 & 0.004 & 0.525 & 0.01 & 206.728 & 4.068 & 18 \\
\hline longitudina & 4.25 & 89.365 & 0.005 & 0.547 & 0.01 & 195.508 & $\begin{array}{r}3.91 \\
\end{array}$ & 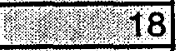 \\
\hline Iongitudina & 5.25 & 89.334 & 0.004 & 0.533 & 0.01 & 205,174 & 4.011 & 18 \\
\hline longitudina & 6.25 & 89.299 & 0.004 & 0.529 & 0.009 & 211.074 & 4078 & Why \\
\hline longitudina & 7.25 & 89.290 & 0.004 & 0.528 & 0.009 & 210.114 & 4.045 & 18 \\
\hline longitudina & 8.25 & 89.293 & 0.005 & 0.59 & 0.011 & 191.142 & 37.708 & (3) \\
\hline Iongitudina & 9.25 & 89.284 & 0.005 & 0.641 & 0.012 & 179.696 & 3.467 & X) \\
\hline longitudina & 10.25 & 89.272 & 0.005 & 0.691 & 0.012 & 175.896 & 3.321 & 18 \\
\hline longitudina & 11.25 & 89.262 & 0.005 & 0.62 & 0.011 & 196.381 & 3.648 & 18 \\
\hline Iongitudina & 0.25 & 89.397 & 0.005 & 0.502 & 0.012 & 149.011 & 3.677 & 18 \\
\hline longitudina & 1.25 & 89.395 & 0.004 & 0.489 & 0.009 & 214.533 & 4.228 & 18 \\
\hline longitudina & 2.25 & 89.376 & 0.004 & 0.501 & 0.009 & 205.115 & 4.143 & 18 \\
\hline longitudina & 3.25 & 89.357 & 0.004 & 0.526 & 0.01 & 203.3 & 4.056 & 18 \\
\hline longitudina & 4.25 & 89.365 & 0.004 & 0.51 & 0.009 & 212.953 & 4.182 & 18 \\
\hline longitudina & 5.25 & 89.350 & 0.004 & 0.51 & 0.009 & 218.725 & 4.222 & 18 \\
\hline longitudina & 6.25 & 89.309 & 0.004 & 0.498 & 0.009 & 221.288 & 4.302 & 18 \\
\hline longitudina & 7.25 & 89.253 & 0.004 & 0.488 & 0.009 & 222.09 & 4.309 & 18 \\
\hline longitudina & 8.25 & 89.236 & 0.004 & 0.498 & 0.009 & 203.007 & 4.125 & 18 \\
\hline longitudina & 9.25 & 89.258 & 0.005 & 0.551 & 0.011 & 187.67 & 3.828 & 18 \\
\hline longitudina & 10.25 & 89.284 & 0.005 & 0.584 & 0.011 & 180.1 & 3.686 & 18 \\
\hline longitudina & 11.25 & 89.330 & 0.005 & 0.656 & 0.012 & 184.067 & 3.482 & 18 \\
\hline longituding & 0.25 & 89.699 & 0.005 & 0.443 & 0.011 & 145.167 & 3.942 & $17 \quad 18$ \\
\hline longitudina & 1.25 & 89.636 & 0.003 & 0.422 & 0.007 & 280.674 & 5.176 & $\begin{array}{l}18 \\
\end{array}$ \\
\hline longitudina & 2.25 & 89.617 & 0.003 & 0.427 & 0.007 & 290.07 & 5.176 & $12 \quad 18$ \\
\hline longitudina & 3.25 & 89.616 & 0.003 & 0.427 & 0.007 & 275.153 & 50.094 & 18 \\
\hline longitudina & 4.25 & 89.612 & 0.003 & 0.417 & 0.007 & 271373 & 5.154 & 18 18 \\
\hline longitudina & 5.25 & 89.614 & 0,003 & 0.434 & $8 \times 10007$ & 261026 & 40.968 & 18.18 \\
\hline longitudina & 6.25 & 89.621 & 0.003 & 0.425 & 0,007 & 260.136 & 0.03 .032 & 18 \\
\hline longitudina & 7.25 & 89619 & 0.003 & 0.431 & 0.007 & 257681 & 4.966 & $\begin{array}{r}18 \\
\end{array}$ \\
\hline Iongitudina & 8.25 & 89.621 & 0.003 & 0.43 & 0.007 & 273.976 & 5.101 & $\begin{array}{r}18 \\
\end{array}$ \\
\hline
\end{tabular}




\begin{tabular}{|c|c|c|c|c|c|c|c|c|}
\hline longitudina & 9.25 & 89.615 & 0.003 & 0.434 & 0.007 & 273.015 & 5.042 & 18 \\
\hline Iongitudina & 10.25 & 89.613 & 0.003 & 0.43 & 0.007 & 280.447 & 5.099 & 18 \\
\hline longitudina & 11.25 & 89.615 & 0.003 & 0.423 & 0.007 & 295.356 & 5.263 & 18 \\
\hline longitudina & 0.25 & 89.501 & 0.006 & 0.425 & 0.013 & 112.17 & 3.644 & 18 \\
\hline Iongitudina & 1.25 & 89.458 & 0.003 & 0.422 & 0.007 & 260.411 & 4.941 & 18 \\
\hline longitudina & 2.25 & 89.451 & 0.003 & 0.43 & 0.007 & 270.048 & 4.932 & 18 \\
\hline longitudina & 3.25 & 89.451 & 0.003 & 0.422 & 0.007 & 266.849 & 5.011 & 18 \\
\hline longitudina & 4.25 & 89.446 & 0.003 & 0.412 & 0.007 & 264.49 & 5.058 & $\overline{18}$ \\
\hline longitudina & 5.25 & 89.435 & 0.003 & 0.423 & 0.007 & 258.289 & 4.946 & 18 \\
\hline longitudina & 6.25 & 89.434 & 0.003 & 0.421 & 0.007 & 255.605 & 4.937 & 18 \\
\hline Iongitudina & 7.25 & 89.421 & 0.003 & 0.426 & 0.007 & 258.62 & 4.916 & 18 \\
\hline Tongitudina & 8.25 & 89.402 & 0.003 & 0.412 & 0.007 & 269.851 & 5.112 & 18 \\
\hline longitudina & 9.25 & 89.391 & 0.003 & 0.42 & 0.007 & 279.572 & 5.075 & 18 \\
\hline Iongitudina & 10.25 & 89.375 & 0.003 & 0.416 & 0.007 & 272.92 & 5.105 & 18 \\
\hline Iongitudina & 11.25 & 89.372 & 0.003 & 0.431 & 0.007 & 279.616 & 5.029 & 18 \\
\hline longitudina & 0.25 & 89.461 & 0.006 & 0.467 & 0.015 & 107.335 & 3.435 & 18 \\
\hline longitudina & 1.25 & 89.413 & 0.003 & 0.439 & 0.008 & 243893 & 4.716 & 178 \\
\hline longitudina & 2.25 & 89.408 & 0.003 & 0.456 & 0.008 & 249419 & 4.661 & 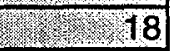 \\
\hline longitudina & 3.25 & 89417 & 0.003 & 0.436 & 0.007 & 251403 & 4808 & $177 \quad 18$ \\
\hline longitudina & 4.25 & 89.406 & 0.003 & 0.438 & 0.008 & 239.875 & 4726 & (1) 18 \\
\hline longitudina & 5.25 & 89.404 & 0.004 & 0.435 & 0.008 & 233.522 & 4.669 & 18 18 \\
\hline Iongitudina & 6.25 & 89.387 & 0.003 & 0.407 & 0.008 & 242.69 & 4.951 & $\begin{array}{l}18 \\
\end{array}$ \\
\hline longitudina & 7.25 & 89.356 & 0.003 & 0.414 & 0.008 & 245.134 & 4.907 & 18 \\
\hline longitudina & 8.25 & 89.322 & 0.003 & 0.432 & 0.008 & 239746 & 4741 & $28 \quad 18$ \\
\hline Iongitudina & 9.25 & 89.330 & 0.004 & 0.471 & 0.009 & 213.663 & 4.366 & 180718 \\
\hline Iongitudina & 10.25 & 89.356 & 0.005 & 0.559 & 0.011 & 189.069 & 3.838 & $1717 \% \quad 18$ \\
\hline Iongitudina & 11.25 & 89.363 & 0.005 & 0.63 & 0.012 & 177504 & 3.542 & 18 \\
\hline Iongitudina & 864 & 89.539 & 0.003 & 0.445 & 0.007 & 260.471 & 4.838 & 18 \\
\hline longitudina & $6+60$ & 89.540 & 0.003 & 0.453 & 0.008 & 251.079 & 4.762 & 18 \\
\hline longitudina & 1056 & 89.532 & 0.003 & 0.452 & 0.007 & 257.28 & 4.784 & 18 \\
\hline longitudina & $17 \times 52$ & 89.514 & 0.003 & 0.44 & 0.007 & 252.333 & 4.822 & 18 \\
\hline longitudina & $4 \% 48$ & 89.528 & 0.003 & 0.41 & 0.007 & 276.351 & 5.184 & 18 \\
\hline longitudina & 1.6.44 & 89.576 & 0.003 & 0.417 & 0.007 & 264.788 & 5.116 & 18 \\
\hline longituding & 10.40 & 89.624 & 0.003 & 0.406 & 0.007 & 282.815 & 5.363 & 18 \\
\hline longitudina & $404-36$ & 89.660 & 0.003 & 0.421 & 0.007 & 269.962 & 5.137 & 18 \\
\hline longitudina & \%8\% $\quad 32$ & 89.669 & 0.003 & 0.418 & 0.007 & 267.025 & 5.159 & 18 \\
\hline longitudina & $3 \quad-28$ & 89.679 & 0.003 & 0.439 & 0.007 & 273.351 & 5.042 & 18 \\
\hline Iongitudina & 424 & 89.683 & 0.003 & 0.432 & 0.008 & 250.026 & 4.929 & 18 \\
\hline longitudina & $5=-20$ & 89.662 & 0.003 & 0.434 & 0.007 & 264.7 & 5.011 & 18 \\
\hline longitudina & 8416 & 89.626 & 0.003 & 0.436 & 0.007 & 266.294 & 5.002 & 18 \\
\hline longitudina & 914 & 89.562 & 0.004 & 0.481 & 0.008 & 237.615 & 4.488 & 18 \\
\hline longitudina & (3) & 89.491 & 0.004 & 0.465 & 0.008 & 242.904 & 4.615 & 18 \\
\hline longitudina & 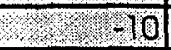 & 89.458 & 0.004 & 0.464 & 0.008 & 230.744 & 4.536 & 18 \\
\hline Iongitudina & -8 & 89.417 & 0.004 & 0.49 & 0.009 & 222.774 & 4.328 & 18 \\
\hline longitudina & (6) 6 & 89.358 & 0.004 & 0.538 & 0.01 & 201.654 & 4.007 & 18 \\
\hline longitudina & 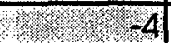 & 89.296 & 0.004 & 0.531 & 0.01 & 203.562 & 4.021 & 18 \\
\hline Iongitudina & 72 & 89.285 & 0.004 & 0.484 & 0.008 & 238.686 & 4.467 & 18 \\
\hline longitudind & 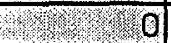 & 89.306 & 0.004 & 0.478 & 0.009 & 228.654 & 4.429 & 18 \\
\hline longitudina & 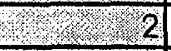 & 89.311 & 0.004 & 0.506 & 0.009 & 210.404 & 4.165 & 18 \\
\hline longituding & 34 & 89.369 & 0.003 & 0.419 & 0.008 & 234.414 & 4.796 & 18 \\
\hline longitudina & 86 & 89.394 & 0.004 & 0.445 & 0.008 & 229.533 & 4.621 & 18 \\
\hline longitudina & $8 \quad 8$ & 89.422 & 0.003 & 0.422 & 0.007 & 252.517 & 4.907 & 18 \\
\hline
\end{tabular}




\begin{tabular}{|l|r|r|r|r|r|r|r|r|}
\hline longitudina & 10 & 89.447 & 0.003 & 0.423 & 0.007 & 261.626 & 4.981 & 18 \\
\hline longitudina & 12 & 89.480 & 0.003 & 0.417 & 0.007 & 267.407 & 5.08 & 18 \\
\hline longitudina & 14 & 89.527 & 0.003 & 0.402 & 0.006 & 282.838 & 5.269 & 18 \\
\hline longitudina & 16 & 89.571 & 0.003 & 0.411 & 0.007 & 263.614 & 5.101 & 18 \\
\hline longitudina & 20 & 89.606 & 0.003 & 0.417 & 0.007 & 257.23 & 5.077 & 18 \\
\hline longitudina & 24 & 89.634 & 0.003 & 0.414 & 0.007 & 262.149 & 5.1 & 18 \\
\hline longitudina & 28 & 89.627 & 0.003 & 0.432 & 0.007 & 254.849 & 4.95 & 18 \\
\hline longitudina & 32 & 89.626 & 0.003 & 0.423 & 0.007 & 263.457 & 5.074 & 18 \\
\hline longitudina & 36 & 89.621 & 0.003 & 0.439 & 0.007 & 267.547 & 4.985 & 18 \\
\hline longitudina & 40 & 89.621 & 0.003 & 0.434 & 0.007 & 266.421 & 5.026 & 18 \\
\hline longitudina & 44 & 89.619 & 0.003 & 0.431 & 0.007 & 260.811 & 5.013 & 18 \\
\hline longitudina & 48 & 89.609 & 0.003 & 0.413 & 0.007 & 270.656 & 5.183 & 18 \\
\hline longitudina & 52 & 89.607 & 0.003 & 0.419 & 0.007 & 260.256 & 5.055 & 18 \\
\hline longitudina & 56 & 89.604 & 0.003 & 0.432 & 0.007 & 259.882 & 4.994 & 18 \\
\hline longitudina & 60 & 89.588 & 0.003 & 0.433 & 0.007 & 252.222 & 4.899 & 18 \\
\hline longitudina & 64 & 89.535 & 0.003 & 0.464 & 0.008 & 248.796 & 4.683 & 18 \\
\hline
\end{tabular}


Ferritic Steel d-zero Comb-like Sample

\begin{tabular}{|c|c|c|c|c|c|c|c|c|}
\hline direction & $\begin{array}{c}\text { Position } \\
\text { (mm) }\end{array}$ & $\begin{array}{c}2 \Theta / \mathrm{d} / \mathrm{a} \\
{[0 / \mathrm{A}]}\end{array}$ & uncertainty & $\begin{array}{c}\text { FWHM } \\
{\left[{ }^{\circ}\right]}\end{array}$ & $\begin{array}{c}\text { uncertainty } \\
{\left[^{\circ}\right]}\end{array}$ & $\begin{array}{c}\text { Peak } \\
\text { Height }\end{array}$ & uncertainty & $\begin{array}{l}\text { sampling } \\
\text { time (min.) }\end{array}$ \\
\hline normal & 0.25 & 89.538 & 0.004 & 0.435 & \begin{tabular}{|r|}
0.01 \\
\end{tabular} & 228.897 & 5.282 & 50 \\
\hline normal & 7.25 & 89.539 & 0.004 & 0.4 & 0.008 & 235.764 & 5.079 & 30 \\
\hline normal & 2.25 & 89.548 & 0.005 & 0.444 & 0.011 & 135.475 & 3.58 & 15 \\
\hline normal & 3.25 & 89.541 & 0.004 & 0.4 & 0.009 & 144.673 & 3.751 & 12 \\
\hline normal & 4.25 & 89.551 & 0.004 & 0.414 & 0.009 & 150.096 & 3.669 & 10 \\
\hline normal & 5.25 & 89.556 & 0.004 & 0.41 & 0.008 & 149.302 & 3.603 & 8 \\
\hline normal & 6.25 & 89.549 & 0.003 & 0.412 & 0.007 & 199.703 & 4.116 & 9 \\
\hline normal & 7.25 & 89.544 & 0.003 & 0.392 & 0.006 & 264.513 & 4.782 & 10 \\
\hline normal & 8.25 & 89.548 & 0.002 & 0.401 & 0.005 & 377.598 & 5.581 & 12 \\
\hline normal & 9.25 & 89.544 & 0.0 .02 & 0.406 & 0.004 & 563.09 & 6.712 & 15 \\
\hline normal & 10.25 & 89.54 & 0.001 & 0.407 & 0.002 & 1384.881 & 10.48 & 30 \\
\hline normal & 11.25 & 89.55 & 0.001 & 0.409 & 0.002 & 3066.647 & 15.334 & 50 \\
\hline normal & 0.25 & 89.523 & 0.006 & 0.707 & 0.014 & 214.435 & 4.125 & 50 \\
\hline normal & 1.25 & 89.54 & 0.005 & 0.592 & 0.012 & 196.562 & 3.994 & 30 \\
\hline normal & 2.25 & 89.537 & 0.005 & 0.482 & 0.01 & 153.624 & 3.603 & 15 \\
\hline normal & 3.25 & 89.543 & 0.004 & 0.465 & 0.009 & 161.303 & 3.643 & 12 \\
\hline normal & 4.25 & 89.545 & 0.004 & 0.457 & 0.009 & 162.81 & 3.605 & 10 \\
\hline normal & 5.25 & 89.559 & 0.004 & 0.444 & 0.008 & 160.361 & 3.573 & 8 \\
\hline normal & 6.25 & 89.559 & 0.003 & 0.447 & 0.007 & 208.072 & 4.055 & 9 \\
\hline normal & 7.25 & 89.562 & 0.003 & 0.43 & 0.006 & 277.222 & 4.699 & 10 \\
\hline normal & 8.25 & 89.567 & 0.002 & 0.442 & 0.005 & 358.559 & 5.259 & 12 \\
\hline normal & 9.25 & 89.564 & 0.002 & 0.446 & 0.004 & 552.232 & 6.402 & 15 \\
\hline normal & 10.25 & 89.564 & 0.001 & 0.436 & 0.002 & 1386.561 & 10.172 & 30 \\
\hline normal & 11.25 & 89.565 & 0.001 & 0.458 & 0.002 & 2471.261 & 13.259 & 50 \\
\hline normal & 0.25 & 89.54 & 0.004 & 0.566 & 0.008 & 359.636 & 5.348 & 50 \\
\hline normal & 1.25 & 89.534 & 0.004 & 0.574 & 0.009 & 272.622 & 4.477 & 30 \\
\hline normal & 2.25 & 89.536 & 0.005 & 0.585 & 0.011 & 157.646 & 3.294 & 15 \\
\hline normal & 3.25 & 89.555 & 0.005 & 0.524 & 0.01 & 157.035 & 3.391 & 12 \\
\hline normal & 4.25 & 89.564 & 0.004 & 0.481 & 0.009 & 157.907 & 3.476 & 10 \\
\hline normal & 5.25 & 89.556 & 0.004 & 0.475 & 0.009 & 147.908 & 3.335 & 8 \\
\hline normal & 6.25 & 89.553 & 0.004 & 0.547 & 0.009 & 178.856 & 3.424 & 9 \\
\hline normal & 7.25 & 89.567 & 0.004 & 0.524 & 0.008 & 220.568 & 3.895 & 10 \\
\hline normal & 8.25 & 89.567 & 0.003 & 0.512 & 0.006 & 326.463 & 4.716 & 12 \\
\hline normal & 9.25 & 89.557 & 0.002 & 0.491 & 0.005 & 508.104 & 5.897 & 15 \\
\hline normal & 10.25 & 89.565 & 0.001 & 0.468 & 0.003 & 1287.528 & 9.541 & 30 \\
\hline normal & 11.25 & 89.559 & 0.001 & 0.481 & 0.002 & 2323.083 & 12.599 & 50 \\
\hline normal & 0.25 & 89.54 & 0.004 & 0.69 & 0.009 & 353.936 & 4.856 & 50 \\
\hline normal & 1.25 & 89.536 & 0.004 & 0.615 & 0.009 & 271.887 & 4.358 & 30 \\
\hline normal & 2.25 & 89.54 & 0.004 & 0.52 & 0.009 & 192.894 & 3.807 & 15 \\
\hline normal & 3.25 & 89.552 & 0.004 & 0.476 & 0.008 & 193.868 & 3.886 & 12 \\
\hline normal & 4.25 & 89.56 & 0.004 & 0.493 & 0.009 & 170.217 & 3.546 & 10 \\
\hline normal & 5.25 & 89.561 & 0.004 & 0.498 & 0.009 & 167.66 & 3.471 & 9 \\
\hline normal & 6.25 & 89.565 & 0.004 & 0.506 & 0.008 & 171.85 & 3.461 & 8 \\
\hline normal & 7.25 & 89.571 & 0.004 & 0.515 & 0.009 & 142.332 & 3.124 & 6 \\
\hline normal & 8.25 & 89.563 & 0.005 & 0.535 & 0.01 & 117.839 & 2.738 & 4 \\
\hline normal & 9.25 & 89.563 & 0.004 & 0.507 & 0.01 & 121.171 & 2.837 & 3.5 \\
\hline normal & 10.25 & 89.569 & 0.004 & 0.485 & 0.009 & 130.517 & 2.979 & 3 \\
\hline
\end{tabular}




\begin{tabular}{|c|c|c|c|c|c|c|c|c|}
\hline normal & 11.25 & 89.561 & 0.004 & 0.486 & 0.009 & 124.399 & 2.891 & 3 \\
\hline normal & 0.25 & 89.515 & 0.005 & 0.772 & 0.012 & 254.806 & 4.133 & 50 \\
\hline normal & 1.25 & 89.528 & 0.005 & 0.692 & 0.012 & 207.405 & 3.734 & 30 \\
\hline normal & 2.25 & 89.544 & 0.005 & 0.511 & 0.01 & 161.192 & 3.593 & 15 \\
\hline normal & 3.25 & 89.558 & 0.004 & 0.513 & 0.01 & 160.601 & 3.46 & 12 \\
\hline normal & 4.25 & 89.574 & 0.004 & 0.483 & 0.009 & 157.745 & 3.464 & 10 \\
\hline normal & 5.25 & 89.568 & 0.004 & 0.49 & 0.008 & 180.347 & 3.621 & 9 \\
\hline normal & 6.25 & 89.562 & 0.004 & 0.483 & 0.008 & 193.237 & 3.747 & 8 \\
\hline normal & 7.25 & 89.562 & 0.004 & 0.471 & 0.008 & 164.167 & 3.428 & 6 \\
\hline normal & 8.25 & 89.562 & 0.004 & 0.47 & 0.009 & 122.331 & 2.961 & 4 \\
\hline normal & 9.25 & 89.558 & 0.004 & 0.444 & 0.008 & 138.063 & 3.194 & 3.5 \\
\hline normal & 10.25 & 89.566 & 0.004 & 0.435 & 0.008 & 149.066 & 3.34 & 3 \\
\hline normal & 11.25 & 89.568 & 0.004 & 0.466 & 0.009 & 128.008 & 3.013 & 3 \\
\hline normal & 0.25 & 89.539 & 0.003 & 0.439 & 0.006 & 434.022 & 6.494 & 50 \\
\hline normal & 1.25 & 89.541 & 0.003 & 0.441 & 0.006 & 350.549 & 5.6 & 30 \\
\hline normal & 2.25 & 89.543 & 0.004 & 0.455 & 0.008 & 215.834 & 4.213 & 15 \\
\hline normal & 3.25 & 89.558 & 0.004 & 0.428 & 0.008 & 196.626 & 4.111 & 12 \\
\hline normal & 4.25 & 89.558 & 0.004 & 0.431 & 0.008 & 183.696 & 3.934 & 10 \\
\hline normal & 5.25 & 89.558 & 0.003 & 0.437 & 0.008 & 190.873 & 3.919 & 9 \\
\hline normal & 6.25 & 89.554 & 0.003 & 0.444 & 0.007 & 199.937 & 3.911 & 8 \\
\hline normal & 7.25 & 89.559 & 0.004 & 0.438 & 0.008 & 168.633 & 3.61 & 6 \\
\hline normal & 8.25 & 89.565 & 0.004 & 0.427 & 0.008 & 132.476 & 3.218 & 4 \\
\hline normal & 9.25 & 89.559 & 0.004 & 0.43 & 0.008 & 140.729 & 3.238 & 3.5 \\
\hline normal & 10.25 & 89.553 & 0.004 & 0.439 & 0.007 & 148.305 & 3.286 & 3 \\
\hline normal & 11.25 & 89.558 & 0.004 & 0.456 & 0.008 & 138.716 & 3.14 & 3 \\
\hline normal & 0.25 & 89.539 & 0.004 & 0.423 & 0.008 & 289.892 & 5.722 & 50 \\
\hline normal & 7.25 & 89.539 & 0.004 & 0.401 & 0.008 & 241.126 & 5.124 & 30 \\
\hline normal & 2.25 & 89.55 & 0.004 & 0.395 & 0.009 & 164.67 & 4.075 & 15 \\
\hline normal & 3.25 & 89.548 & 0.004 & 0.415 & 0.009 & 162.956 & 3.839 & 12 \\
\hline normal & 4.25 & 89.546 & 0.004 & 0.404 & 0.008 & 169.894 & 3.898 & 10 \\
\hline normal & 5.25 & 89.557 & 0.003 & 0.417 & 0.008 & 183.573 & 3.952 & 9 \\
\hline normal & 6.25 & 89.549 & 0.003 & 0.401 & 0.007 & 203.548 & 4.168 & 8 \\
\hline normal & 7.25 & 89.558 & 0.003 & 0.413 & 0.007 & 174.801 & 3.74 & 6 \\
\hline normal & 8.25 & 89.554 & 0.004 & 0.416 & 0.008 & 134.133 & 3.249 & 4 \\
\hline normal & 9.25 & 89.544 & 0.004 & 0.401 & 0.007 & 142.627 & 3.381 & 3.5 \\
\hline normal & 10.25 & 89.542 & 0.003 & 0.397 & 0.007 & 151.23 & 3.468 & 3 \\
\hline normal & 71.25 & 89.553 & 0.003 & 0.4 & 0.006 & 181.656 & 3.777 & 3 \\
\hline
\end{tabular}




\begin{tabular}{|c|c|c|c|c|c|c|c|c|c|c|c|c|}
\hline$\angle \varepsilon$ & LEt & LE & 699 & $s t$ & $9+1$ & $\varepsilon 01$ & LE6 & $0 L$ & $\angle Z \angle L$ & Tot1 & 999- & Tgz'l1 \\
\hline$\underline{G Z}$ & $08 \varepsilon$ & 82 & $99 \varepsilon$ & $9 \varepsilon$ & 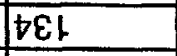 & 69 & 9201 & $D L$ & 986 & III & $86 \varepsilon^{-}$ & 9201 \\
\hline$s \varepsilon$ & 092 & $1 \varepsilon$ & $\varepsilon 6$ & $\hbar \varepsilon$ & 99 & 001 & $t 76$ & $\varepsilon 8$ & $0 z^{-}$ & 96 & $6 \angle L^{-}$ & 92.6 \\
\hline $0 \varepsilon$ & 212 & $\Phi \varepsilon$ & $8 L^{-}$ & $\angle \varepsilon$ & 80 & $9 L$ & 186 & 101 & L69- & LL & $2 \varepsilon$ & 92.8 \\
\hline $9 \varepsilon$ & $8<1$ & $\varepsilon \mathcal{Z}$ & $E \mathcal{E l -}$ & $\varepsilon 己$ & St & StL & $\nabla \angle 6$ & IS & $986^{-}$ & 99 & $5 \varepsilon$ & $92 \mathrm{~L}$ \\
\hline$\angle 1$ & 991 & $\angle 2$ & $\angle 9 l^{-}$ & st & $8-$ & $0 t$ & $1 \angle 6$ & 98 & 196- & 92 & $9 \varepsilon^{-}$ & $S Z^{\circ} 9$ \\
\hline it & $D L Z$ & $\angle Z$ & $1.1-$ & $\varepsilon z$ & $6^{-}$ & $\varepsilon \varepsilon 1$ & ELLL & 79 & $\angle 9 L^{-}$ & $z t$ & $6 \angle 1-$ & SZ:S \\
\hline 19 & $2 E 2$ & $9 t$ & $89^{-}$ & $L E$ & $\angle Z^{-}$ & 991 & 1911 & 621 & ESS- & $t s$ & $97 \varepsilon^{-}$ & g2't \\
\hline $9 \varepsilon$ & 082 & 62 & 8 & 12 & Sl- & 711 & 9921 & 19 & $91 \varepsilon^{-}$ & $2 b$ & $b s b-$ & $S Z^{\prime} \varepsilon$ \\
\hline 89 & 692 & $2 t$ & $\angle Z$ & $0 t$ & $00^{-}$ & 912 & $0<11$ & 86 & $\varepsilon<1-$ & 28 & 699- & GZ'Z \\
\hline $0 L$ & $\angle 0 \varepsilon$ & $9 \varepsilon$ & StL & $\angle \varepsilon$ & $g 2$ & 622 & 1811 & $\nabla S$ & $\angle 9$ & 99 & tSt- & GZ'! \\
\hline$\angle O L$ & $2 \angle 2$ & $0 L$ & 911 & 19 & 9 & $\varepsilon+\varepsilon$ & $t \rightarrow 0 t$ & $0 \angle 1$ & $\angle D I$ & StL & $68 t^{-}$ & $9 Z^{\prime} 0$ \\
\hline 10112 & |Eu!pny!buo| & $1011 \theta$ & OSJOASUEAf & 10110 & jemsou & $10 \mathrm{~d} \theta$ & | & $1011 \theta$ & әSIOASUEA & 10110 & jemsou & ${ }_{*}(\mathrm{uw})$ \\
\hline
\end{tabular}

\begin{tabular}{|c|c|c|c|c|c|c|c|c|c|c|c|c|}
\hline $9 t$ & $\downarrow 9 z^{-}$ & $\angle 2$ & SE- & to & $0 L^{-}$ & $6 \varepsilon 1$ & LOOL- & $9 \varepsilon$ & $\varepsilon z \varepsilon$ & $\varepsilon \varepsilon 1$ & 69 & $9 Z^{\prime \prime 11}$ \\
\hline st & $00 z^{-}$ & 82 & $11-$ & $0 b$ & $79^{-}$ & $8 \varepsilon 1$ & $06 L^{-}$ & 29 & sog & $\angle 11$ & $\varepsilon^{-}$ & 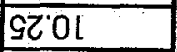 \\
\hline ZE & $G \angle L^{-}$ & 02 & $L$ & $0 \varepsilon$ & st- & 66 & $6 \mathrm{ZL}^{-}$ & $\Phi \varepsilon$ & SzE & $\angle 8$ & $\varepsilon z$ & 926 \\
\hline$\varepsilon \varepsilon$ & $81{ }^{-}$ & 62 & $\angle 9$ & 92 & 6 & 101 & $119-$ & 09 & $66 \varepsilon$ & $\angle 9$ & 021 & $9 Z^{\prime} 8$ \\
\hline 20 & $6 \varepsilon l^{-}$ & $\varepsilon Z$ & 72 & $\angle 2$ & $\mathrm{HL}^{-}$ & $9 \varepsilon 1$ & $989-$ & $0 t$ & $80 \varepsilon$ & 99 & $\varepsilon 01$ & $G Z L$ \\
\hline$\nabla \varepsilon$ & EZL- & 91 & $\nabla \varepsilon$ & 12 & I- & $1+1$ & 689- & 91 & $\angle L \varepsilon$ & $6 t$ & $71-$ & $92 \%$ \\
\hline$\varepsilon D$ & LOL- & 62 & 09 & $D Z$ & 02 & $\angle E L$ & SSS- & $D L$ & $G \angle \varepsilon$ & $\varepsilon t$ & 001 & $G Z^{\circ} 9$ \\
\hline SE & DOL- & $2 Z$ & $\varepsilon S$ & 02 & $\varepsilon 己$ & $1+1$ & E99- & $\varepsilon g$ & $\varepsilon+\varepsilon$ & $0 t$ & $0 \angle 1$ & $g Z^{\prime} D$ \\
\hline$\angle 9$ & LLL- & Et & $\varepsilon t$ & $2 t$ & 22 & $\forall<1$ & $629-$ & 711 & $\varepsilon z \varepsilon$ & $\angle O 1$ & 202 & $9 Z: \varepsilon$ \\
\hline 00 & $\varepsilon 8^{-}$ & $t z$ & $8 L$ & 61 & 89 & $0 \varepsilon t$ & 679 & 79 & $18 \varepsilon$ & 81 & 792 & $G Z^{\prime} Z$ \\
\hline G己 & ELt- & $\angle 1$ & $0 S$ & 61 & $0 \varepsilon$ & 82 & 809- & $0 t$ & $\nabla \varepsilon \varepsilon$ & $8 t$ & $\angle 12$ & $9 Z^{\prime} 1$ \\
\hline 97 & LH- & $\nabla \varepsilon$ & ot & 62 & $\varepsilon S$ & 901 & $619^{-}$ & 06 & 892 & 29 & BZE & $92^{\prime} 0$ \\
\hline 10110 & |eu!pn!!̣uo| & 10110 & $\partial \mathrm{\theta s \lrcorner} \wedge \wedge$ sued & $1011 \theta$ & jeusou & دOدرO & |eu!pn!़̣uo| & & әsıəภsued? & 10110 & jemsou & ${ }_{*}(u(u) 1$ \\
\hline & & & & $(e d n$ & 1) sassans & & & & & (u!̣edtSOdo! & !u) suleds & I әu! \\
\hline
\end{tabular}
E.0
o!̣es uo!ssod
GZZ
(edפ) $\exists$

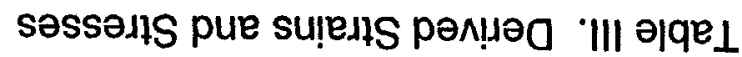




\begin{tabular}{|c|c|c|c|c|c|c|c|c|c|c|c|c|}
\hline 21 & $\angle 6 \varepsilon$ & $b z$ & 29 & LI & $\angle 0^{-}$ & 61 & $90 \angle 1$ & $6 L$ & 06L- & $\mathrm{s}$ & ZZ8- & 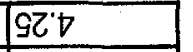 \\
\hline 02 & 199 & $0 z$ & $16 \varepsilon$ & 22 & $\varepsilon 8$ & $\angle G$ & 2981 & $\varepsilon S$ & 088 & 79 & $106^{-}$ & $G Z^{\prime} \varepsilon$ \\
\hline$g \varepsilon$ & 629 & It & $1 \nabla \varepsilon$ & $2 t$ & 221 & 16 & $s \varepsilon \angle 1$ & $\angle 1 L$ & $9+9$ & 221 & $819-$ & $G Z^{\prime} Z$ \\
\hline $0 \varepsilon$ & $68 \varepsilon$ & $2 t$ & 11L & $\varepsilon 己$ & 16 & $6 L$ & 8stl & $9 \varepsilon 1$ & 9tL- & $9 \varepsilon$ & $192-$ & $G Z^{\prime} 1$ \\
\hline 82 & $00 \varepsilon$ & 99 & 02 & $8 \varepsilon$ & $9 \varepsilon$ & $2 \varepsilon$ & 6921 & 081 & $\angle G \varepsilon^{-}$ & 86 & $Z \angle Z^{-}$ & $9 Z^{\prime} 0$ \\
\hline 10110 & |eu!pn!̣buol & 10110 & 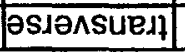 & 10110 & peسnou & $1011 \theta$ & |eu!pnı!6uol & & כ| & $\operatorname{dod1} \theta$ & pemsou & ${ }_{*}(u \omega) 1$ \\
\hline
\end{tabular}

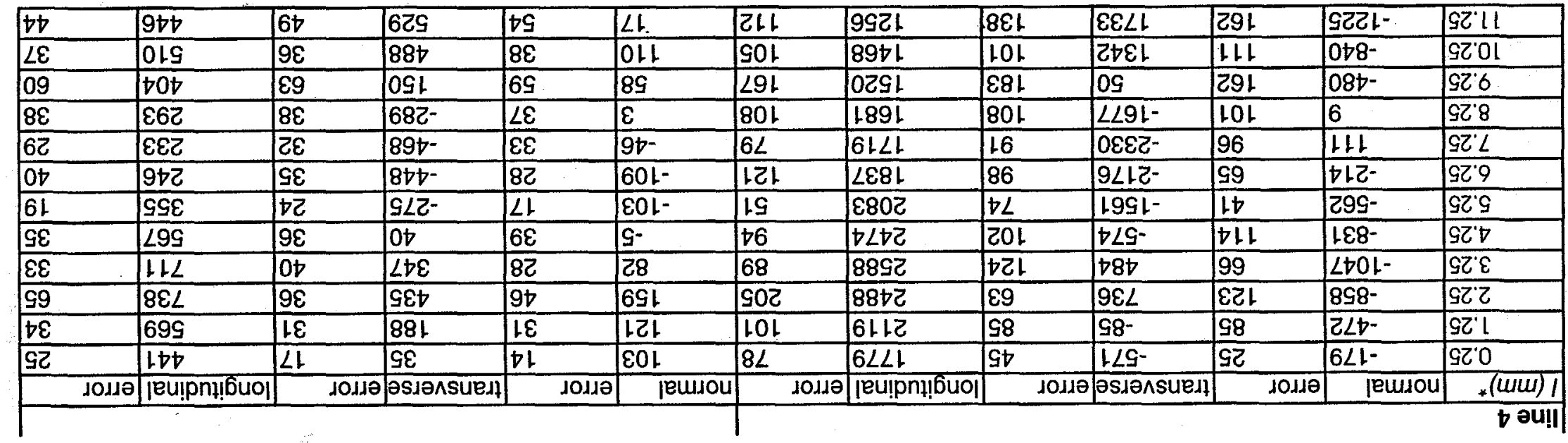

\begin{tabular}{|c|c|c|c|c|c|c|c|c|c|c|c|c|}
\hline$\angle \varepsilon$ & $0 \angle t$ & दो & 829 & $9 t$ & EOL & 96 & 91トL & 811 & 9202 & $\angle E L$ & $9001^{-}$ & 9211 \\
\hline $2 D$ & 109 & $s \varepsilon$ & $\angle Z G$ & $\angle \varepsilon$ & $\angle 91$ & $\varepsilon Z$ & LOEL & 86 & $\varepsilon s+1$ & 701 & $629^{-}$ & $9 Z^{\prime} \mathrm{Ol}$ \\
\hline $9 \varepsilon$ & $2 \angle \varepsilon$ & 22 & 081 & 62 & 66 & EトL & ह8ट। & 2t & $0 \angle 1$ & 28 & $962-$ & $9 z^{\prime} 6$ \\
\hline $0 t$ & $00 \varepsilon$ & 02 & $\varepsilon \angle L^{-}$ & 02 & $9 \varepsilon$ & $2 \varepsilon 1$ & $\nabla \backslash S \mid$ & 82 & 9LZ।- & $\overline{s \varepsilon}$ & $6-$ & 928 \\
\hline 61 & $6 \pitchfork 2$ & $\angle t$ & $88 \varepsilon^{-}$ & $\varepsilon$ & $6 t^{-}$ & $\angle G$ & 6891 & $\angle t$ & $2661^{-}$ & 82 & $2 \varepsilon^{-}$ & $S^{\prime} L$ \\
\hline 92 & $\nabla 9 \mathcal{Z}$ & $\varepsilon$ & $90 b^{-}$ & 61 & 6IL- & 28 & 6281 & 9 & $8 \angle 6 L^{-}$ & $0 S$ & LEE- & $9 Z^{\prime} 9$ \\
\hline$\angle 2$ & $\overline{99 \varepsilon}$ & $b z$ & $6 \varepsilon z^{-}$ & 62 & O1. & $9 L$ & 8802 & 09 & EOtL- & 98 & 999- & g'? \\
\hline 99 & $19 t$ & 92 & $b z^{-}$ & 69 & $2 L^{-}$ & $1+1$ & $\angle L L Z$ & tहZ & 929- & $\angle S I$ & $106^{-}$ & $9 Z^{\circ} D$ \\
\hline$L E$ & 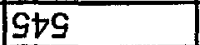 & 82 & SSt & $\angle L$ & 9 & 96 & $\angle O Z Z$ & 28 & $\angle \nabla^{\circ}$ & 71 & $\angle 06^{-}$ & $g Z^{\prime} \varepsilon$ \\
\hline$\varepsilon S$ & $\not O S$ & $0 t$ & SEL & $\angle \varepsilon$ & 99- & tol & $9+12$ & SOL & 81 & 88 & ந七レட- & $s Z^{\prime} Z$ \\
\hline $1 t$ & $\angle S G$ & $\varepsilon Z$ & $9 \angle 1$ & 12 & $9 k^{-}$ & $\nabla \varepsilon t$ & t9Z2 & $\angle t$ & 29 & $\nabla \varepsilon$ & 1901- & $9 Z^{\prime} 1$ \\
\hline 02 & $6 \nabla 9$ & 92 & 901 & $\varepsilon z$ & 0 & 19 & 6622 & 08 & $19 Z^{-}$ & 79 & $9 \angle 8^{-}$ & $9 Z^{\circ} 0$ \\
\hline 10110 & |eu!pnı!buol & & 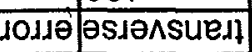 & 10110 & jemsou & 10110 & |eu!pny!buo| & & 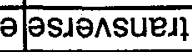 & 10110 & jemsou & ${ }_{*}(u \omega u) 1$ \\
\hline
\end{tabular}




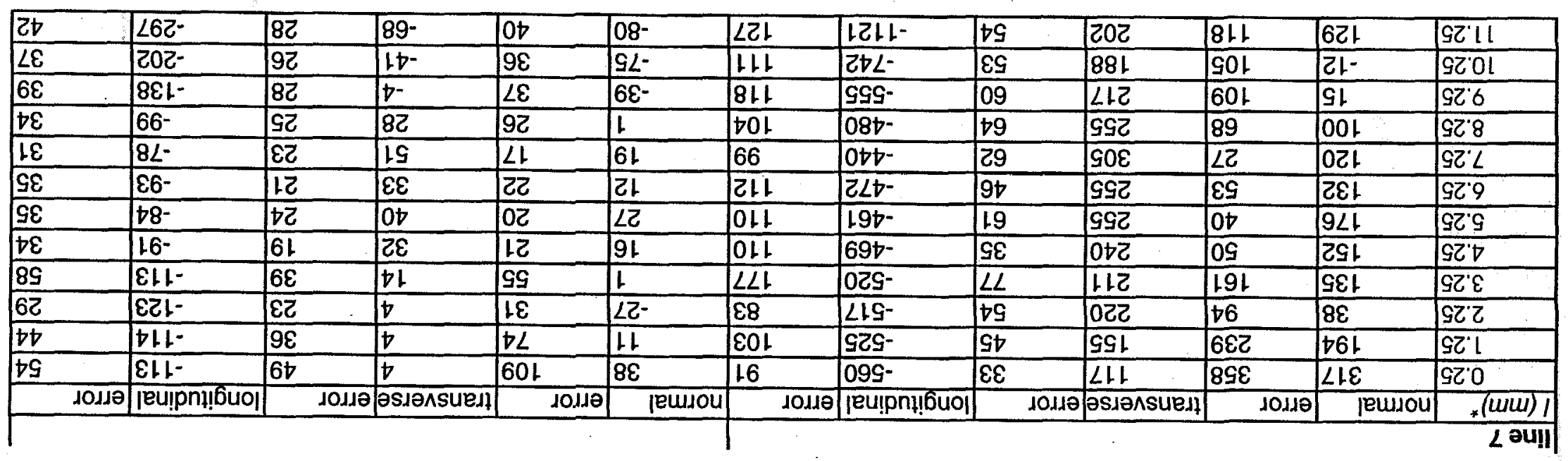

\begin{tabular}{|c|c|c|c|c|c|c|c|c|c|c|c|c|}
\hline LS & 761 & $L E$ & $10 \varepsilon$ & 60 & $2 t^{-}$ & 991 & $\angle L S$ & $D G$ & $9 \varepsilon 1 t$ & $\angle Z I$ & $978-$ & 9211 \\
\hline $6 \varepsilon$ & $\angle \varepsilon Z$ & $s \varepsilon$ & $8 \angle 1$ & It & 2 & 211 & 918 & 86 & $2 \angle b$ & 811 & stg- & 9201 \\
\hline $0 \varepsilon$ & $\angle 12$ & 82 & $\angle \varepsilon$ & $\varepsilon \varepsilon$ & $t 2$ & 78 & 988 & $9 L$ & $891-$ & $\angle 6$ & IEZ- & SZ'6 \\
\hline $7 \varepsilon$ & $\angle 12$ & $2 \varepsilon$ & $E 9^{-}$ & 62 & $\varepsilon 9$ & 001 & 996 & 16 & $\angle 69^{-}$ & $8 L$ & 81 & SZ'8 \\
\hline$D 1$ & 191 & S1 & Stl- & $g 1$ & $L^{-}$ & $6 \varepsilon$ & $\angle 16$ & $\varepsilon t$ & $298^{-}$ & 26 & 0s- & $G Z^{\prime} L$ \\
\hline $1 \varepsilon$ & $6 \varepsilon t$ & $\varepsilon z$ & $9 \angle L^{-}$ & 62 & b9- & 26 & $9 \varepsilon 6$ & $\varepsilon s$ & $8 \angle 8-$ & 98 & $\angle \varepsilon Z^{-}$ & $9 Z^{\prime} 9$ \\
\hline $8 \varepsilon$ & 991 & 92 & 621- & $S t$ & 68- & 601 & 9201 & 16 & $\varepsilon<9^{-}$ & $6 \varepsilon 1$ & sto- & GZ' 9 \\
\hline$\angle Z$ & $\varepsilon z Z$ & 81 & $2 b^{-}$ & 52 & $08-$ & 78 & tSLL & $8 \varepsilon$ & $8 \angle \varepsilon^{-}$ & $0 L$ & $\angle 6 S^{-}$ & $9 Z^{\prime} t$ \\
\hline 6 & 682 & 91 & 29 & 21 & $1 b^{-}$ & 81 & 9921 & 09 & 99- & $L E$ & 099- & $92 \varepsilon$ \\
\hline 16 & $\nabla 2 \varepsilon$ & $6 \varepsilon$ & 011 & 16 & 22 & 911 & 7921 & $\angle O L$ & 92 & 811 & $08 t^{-}$ & $\mathcal{G Z} Z^{\prime}$ \\
\hline 61 & $8 \angle \varepsilon$ & 12 & $\angle G L$ & $7 t$ & 601 & G9 & bZEL & 79 & 09 & 92 & $622^{-}$ & GZ! \\
\hline$\varepsilon \varepsilon$ & $\angle Z t$ & 22 & $s<t$ & $\angle \varepsilon$ & 702 & 96 & $16 \varepsilon 1$ & $\varepsilon \varepsilon$ & $69-$ & Stl & 901 & 920 \\
\hline $1011 \theta$ & |eu!pn & 10110 & כstənsuedt & 10112 & pewiou & $1011 \theta$ & |eu!pn!̣buo| & JOLJO & әsıәภsued & 10110 & jemiou & ${ }_{*}(u \omega) 1$ \\
\hline
\end{tabular}

\begin{tabular}{|c|c|c|c|c|c|c|c|c|c|c|c|c|}
\hline 09 & $\angle 9 \varepsilon$ & $8 \varepsilon$ & 087 & 86 & 22 & 801 & 096 & 88 & S19L & 001 & $080 L^{-}$ & 9211 \\
\hline$g \varepsilon$ & $60 t$ & $2 \varepsilon$ & $\varepsilon \neq \varepsilon$ & $2 b$ & $6 L$ & $\angle 6$ & 9921 & 08 & $D \angle 8$ & $\angle Z L$ & $\varepsilon 99^{-}$ & $9 Z^{\circ} 01$ \\
\hline$\varepsilon \varepsilon$ & $L Z \varepsilon$ & $\angle Z$ & $9 L$ & $\angle \varepsilon$ & 18 & 56 & $912 t$ & 29 & $66 \mathrm{~L}^{-}$ & 211 & $\angle 91^{-}$ & 926 \\
\hline $2 \varepsilon$ & 212 & $6 \varepsilon$ & $802^{-}$ & टह & 09 & 88 & $2 \varepsilon+1$ & 611 & 99Z1- & $\angle 8$ & SS2 & $9 Z^{\prime} 8$ \\
\hline 02 & 981 & $t 2$ & $91 \varepsilon^{-}$ & $\angle 1$ & $\varepsilon \varepsilon$ & 99 & $66 \mathrm{tt}$ & $g L$ & $9691-$ & $2 t$ & $22 \varepsilon$ & $G Z^{\prime} L$ \\
\hline $2 \varepsilon$ & 981 & $6 \varepsilon$ & $+1 \varepsilon^{-}$ & 27 & $9^{-}$ & $6 L$ & ESZL & 01.1 & $7 \varepsilon 9 \mathrm{~L}^{-}$ & 921 & $t+1$ & $9 Z^{\prime} 9$ \\
\hline 16 & $\varepsilon \mathcal{E} Z$ & 96 & bZZ- & $9 \varepsilon$ & $6 L^{-}$ & GLt & 1001 & $0+1$ & $66 \mathrm{Lt}^{-}$ & 06 & $99 \varepsilon^{-}$ & $9 Z^{\prime} G$ \\
\hline
\end{tabular}




\begin{tabular}{|c|c|c|c|c|c|c|c|c|c|c|c|c|c|}
\hline$\nabla \varepsilon$ & & $06-$ & 81 & th & $b 2$ & 69 & 601 & $\angle D S-$ & 82 & 112 & $\varepsilon 9$ & $\varepsilon \angle \varepsilon$ & $2 \varepsilon$ \\
\hline $8 t$ & & $00 \mathrm{~L}^{-}$ & $5 \varepsilon$ & $6 \varepsilon$ & 82 & 79 & ISt & 989- & 76 & $2 Z Z$ & 09 & $89 \varepsilon$ & 82 \\
\hline 00 & & $66^{-}$ & 81 & 99 & $2 Z$ & $9 L$ & $1 \varepsilon L$ & $719-$ & 11 & $8 \angle 2$ & 80 & $76 \varepsilon$ & $7 \mathrm{Z}$ \\
\hline$\varepsilon \varepsilon$ & & EL!- & 81 & $0 t$ & $\nabla 2$ & 89 & 701 & $9+9-$ & $9 z$ & $\angle \varepsilon Z$ & 99 & $00 t$ & 02 \\
\hline $2 t$ & & $S \varepsilon^{-}$ & 12 & $2 L$ & 12 & 101 & $6 \varepsilon 1$ & $68 \varepsilon^{-}$ & $0 \varepsilon$ & $\nabla \varepsilon \mathcal{Z}$ & $\angle \varepsilon$ & 100 & 91 \\
\hline $6 \varepsilon$ & & $z \varepsilon$ & 62 & 16 & 12 & $0 \varepsilon 1$ & SZL & $t G 1^{-}$ & $8 L$ & $\angle 81$ & $\angle Z$ & 916 & 71 \\
\hline 62 & & S1t & 92 & 88 & $b 2$ & SG1 & $\angle 8$ & $\angle 81$ & $2 L$ & $2 \varepsilon$ & 89 & $61 t$ & 21 \\
\hline 62 & & 761 & 12 & 09 & 61 & 691 & 68 & $\angle S G$ & $\varepsilon 9$ & $\angle 1 Z^{-}$ & $\angle t$ & ElD & 01 \\
\hline 62 & & $\varepsilon+2$ & $\angle 2$ & 61 & 02 & 891 & $\angle 8$ & 188 & $8 L$ & $89 t^{-}$ & $t b$ & $86 \varepsilon$ & 8 \\
\hline 02 & & 892 & 61 & $62^{-}$ & 02 & 111 & 69 & $8 \varepsilon 0 \mathrm{~L}$ & 29 & $129-$ & 99 & $\angle 81$ & 9 \\
\hline 62 & & $8 \angle 2$ & 91 & E6- & IZ & IS & 26 & 1621 & $\angle Z$ & $298^{-}$ & $\angle 9$ & $8 \mathrm{t}^{-}$ & $t$ \\
\hline $9 \varepsilon$ & & $\underline{G Z \varepsilon}$ & $9 \varepsilon$ & SEl- & $1 \varepsilon$ & 29 & 501 & $1+S t$ & DOL & 9レト- & $6 L$ & $\varepsilon \mathcal{E}$ & 6 \\
\hline $9 \varepsilon$ & & $\angle Z D$ & $6 \varepsilon$ & $8 \angle L^{-}$ & $\nabla \varepsilon$ & 701 & 001 & 7661 & $\varepsilon L$ & $\angle 6 t L^{-}$ & 16 & $|E|$ & D \\
\hline $8 \varepsilon$ & & $80 t$ & $\angle \varepsilon$ & $261-$ & 92 & ESL & $\varepsilon 11$ & $2 \neq 0 z$ & 601 & 2991- & $t g$ & $\angle E \varepsilon$ & $\mathrm{z}^{\circ}$ \\
\hline $1 Z$ & & 880 & $\varepsilon \varepsilon$ & $\angle L L^{-}$ & $1 \varepsilon$ & $s<t$ & $6 \varepsilon$ & 0912 & 201 & $699 L^{-}$ & 68 & $69 \varepsilon$ & $f^{2}$ \\
\hline$\varepsilon z$ & & $0 t 0$ & 02 & $681-$ & $\varepsilon 2$ & 001 & $\angle 9$ & $2 \angle 0 Z$ & ZS & 6991- & 89 & 111 & 9 - \\
\hline$s \varepsilon$ & & $01 \varepsilon$ & $\underline{G Z}$ & $902^{-}$ & 92 & 92 & $0+1$ & 9191 & $\varepsilon 9$ & Egع - & 99 & $t z^{-}$ & $8^{-}$ \\
\hline $6 \varepsilon$ & & $\varepsilon 81$ & $8 \varepsilon$ & $8 \angle L-$ & $b 2$ & $L^{-}$ & $\angle L$ & 2901 & st1 & $\angle 20 L^{-}$ & $\angle \varepsilon$ & $6 \varepsilon^{-}$ & 01 \\
\hline $9 \varepsilon$ & & $\varepsilon+1$ & 02 & Ez!- & 61 & $\varepsilon \varepsilon$ & 811 & $9 S L$ & $6 \varepsilon$ & $28 L^{-}$ & $L E$ & 021 & 21 \\
\hline$\angle t$ & & 101 & $1 \varepsilon$ & $66^{-}$ & SE & $2 \varepsilon$ & OSL & $8 \varepsilon 9$ & 92 & 919- & $2 t$ & $\angle E L$ & $\$ 1$ \\
\hline Lt & & $\underline{G \varepsilon}$ & $\angle Z$ & $\angle 8^{-}$ & $s \varepsilon$ & $S z$ & $\angle Z L$ & $6 \varepsilon 2$ & $t g$ & $99 t^{-}$ & 001 & 181 & 91 \\
\hline $9 \varepsilon$ & & $68-$ & 22 & $2 L^{-}$ & 22 & टह & S1t & $81 \varepsilon^{-}$ & 60 & $6+2^{-}$ & 19 & $8 † \varepsilon$ & $B c^{-}$ \\
\hline $2 t$ & & E91- & 22 & $6 \varepsilon^{-}$ & 12 & $\nabla \varepsilon$ & $\angle \varepsilon L$ & $02 L^{-}$ & 20 & 0 & $2 \varepsilon$ & $22 t$ & $7 \mathrm{Z}$ \\
\hline 20 & & $26 \mathrm{~L}^{-}$ & 92 & 12 & 22 & $9 t$ & $9 \varepsilon 1$ & $0+6^{-}$ & $\varepsilon 9$ & $\angle 82$ & $\nabla \varepsilon$ & LEt & $8 \mathrm{Z}^{-}$ \\
\hline $8 \varepsilon$ & & $60 z^{-}$ & 92 & 22 & $0 \varepsilon$ & to & 911 & $8101^{-}$ & 69 & $91 \varepsilon$ & 08 & $\angle t \theta$ & $80^{-2}$ \\
\hline $8 \varepsilon$ & & $06 \mathrm{~L}^{-}$ & 22 & $9 t$ & 02 & 99 & 221 & $766^{-}$ & st & $69 \varepsilon$ & $\Phi \varepsilon$ & 980 & 96 \\
\hline $8 \varepsilon$ & & $99 l^{-}$ & $t 2$ & 69 & 12 & 78 & 121 & $826^{-}$ & 09 & $2 \angle \varepsilon$ & $9 \varepsilon$ & $\angle 19$ & Ot. \\
\hline $9 \varepsilon$ & & $86^{-}$ & $\angle 2$ & 001 & GZ & $\varepsilon 己$ & $\angle O L$ & $\nabla \varepsilon L^{-}$ & $L$ & $\varepsilon \vdash t$ & $\varepsilon 9$ & 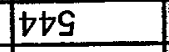 & $7 D^{\circ}$ \\
\hline 00 & & $L^{-}$ & $\Phi \varepsilon$ & 011 & $\angle \varepsilon$ & $8+1$ & $\angle 1+$ & $9 \angle \varepsilon^{-}$ & 56 & $20 \varepsilon$ & 801 & $0 Z 9$ & $8 \mathrm{t}$ \\
\hline$\overline{G \varepsilon}$ & & 88 & $b 2$ & $\varepsilon 2 t$ & $0 \varepsilon$ & 191 & 801 & 11 & 19 & 612 & 98 & $9 \varepsilon t$ & 69 \\
\hline$\varepsilon \varepsilon$ & & SEL & 22 & 201 & 12 & $\angle \nabla t$ & SOL & 892 & 59 & $6 L$ & $\angle t$ & $\angle \varepsilon \varepsilon$ & $99=$ \\
\hline 00 & & 601 & 92 & 29 & $0 \varepsilon$ & 86 & $\angle Z I$ & 982 & 99 & $b t^{-}$ & 08 & 612 & $109^{\circ}$ \\
\hline $9 \varepsilon$ & & 09 & 92 & 61 & $6 \mathrm{t}$ & 69 & $2+1$ & 091 & $\angle 9$ & $7 L^{-}$ & 82 & $69 t$ & 199 \\
\hline & I0נ12 & jeu!pn!!buo| & & 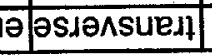 & 10.110 & jeunou & $1011 \theta$ & |eu!pnı! & $1011 \theta$ & 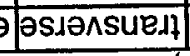 & i) & Ty & (um) $p$ \\
\hline
\end{tabular}




\begin{tabular}{|c|c|c|c|c|c|c|c|c|c|c|c|c|}
\hline $9 \varepsilon$ & 221 & 82 & $0 \varepsilon^{-}$ & $6 \varepsilon$ & 88 & 901 & $99 t$ & 29 & $\varepsilon\left\llcorner b^{-}\right.$ & 811 & 692 & 70 \\
\hline $9 \varepsilon$ & $\varepsilon z$ & $\varepsilon \varepsilon$ & $0 t^{-}$ & $\varepsilon z$ & 92 & 111 & $2 Z 1$ & 96 & ItbZ- & $s t$ & $\nabla \varepsilon L$ & 09 \\
\hline 92 & $80 \mathrm{~K}^{-}$ & 12 & $18^{-}$ & $D 1$ & $\angle b^{-}$ & 88 & $90 \varepsilon^{-}$ & 89 & 9GL- & 11 & $2 t$ & 99 \\
\hline $2 \varepsilon$ & $\angle O L^{-}$ & 92 & $9 \varepsilon-$ & 02 & $\angle-$ & 001 & $91 b^{-}$ & $L$ & $6^{-}$ & $\varepsilon t$ & 691 & 29 \\
\hline 62 & $18^{-}$ & 61 & $\angle 1$ & 02 & 86 & 06 & $s t t^{-}$ & 96 & 021 & 80 & $\angle 6 Z$ & 81 \\
\hline $1 \varepsilon$ & $E L^{-}$ & 61 & $6 t$ & 81 & $\varepsilon L$ & 001 & $88 t^{-}$ & 26 & 612 & $\angle E$ & $\angle 9 \varepsilon$ & 60 \\
\hline Et & $78-$ & 92 & st & 92 & $0 L$ & $0+1$ & 9Z9- & OS & 612 & $0 S$ & $\varepsilon 9 \varepsilon$ & $0 b$ \\
\hline $0 t$ & $06^{-}$ & 92 & $\angle D$ & $b 2$ & $2 L$ & 821 & 899- & 89 & IEZ & $\varepsilon g$ & $6 \angle \varepsilon$ & $9 \varepsilon$ \\
\hline
\end{tabular}


Table IV. Measured gauge volume size for individual detectors

\begin{tabular}{|l|c|c|c|c|c|c|c|}
\hline Detector No. & 1 & 2 & 3 & 4 & 5 & 6 & 7 \\
\hline $\begin{array}{l}\text { Gauge volume } \\
\text { size }\left(\mathrm{mm}^{2}\right)\end{array}$ & $1.7 \times 2$ & $1.7 \times 2$ & $1.7 \times 2$ & $1.7 \times 2$ & $1.7 \times 1.9$ & $1.6 \times 1.9$ & $1.6 \times 1.9$ \\
\hline
\end{tabular}

